\title{
Current Personnel Dosimetry Practices at DOE Facilities
}

J. J. Fix, PNL Principal Investigator

J. M. Selby, PNL Project Manager

E. J. Vallario, DOE Project Manager

May 1981

Prepared for the U.S. Department of Energy under Contract DE-AC06-76RLO 1830

Pacific Northwest Laboratory

Operated for the U.S. Department of Energy by Battelle Memorial Institute 


\title{
NOTICE
}

This report was prepared as an account of work sponsored by the United States Government. Neither the United States nor the Department of Energy, nor any of their employees, nor any of their contractors, subcontractors, or their employees, makes any warranty, express or implied, or assumes any legal liability or responsibility for the accuracy, completeness or usefulness of any information, apparatus, product or process disclosed, or represents that its use would not infringe privately owned rights.

The views, opinions and conclusions contained in this report are those of the contractor and do not necessarily represent those of the United States Government or the United States Department of Energy.

\section{PACIFIC NORTHWEST LABORATORY operated by \\ BATTELLE \\ for the \\ UNITED STATES DEPARTMENT OF ENERGY \\ Under Contract DE-AC06-76RLO 1830}

\author{
Printed in the United States of America \\ Available from \\ National Technical Information Service \\ United States Department of Commerce \\ 5285 Port Royal Road \\ Springfield, Virginia 22151
}

Price: Printed Copy $\$$ $\because$ Microfiche $\$ 3.00$

$\begin{array}{cr}\text { •Pages } & \begin{array}{c}\text { NTIS } \\ \text { Selling Price }\end{array} \\ 001-025 & \$ 4.00 \\ 026-050 & \$ 4.50 \\ 051-075 & \$ 5.25 \\ 076-100 & \$ 6.00 \\ 101-125 & \$ 6.50 \\ 126-150 & \$ 7.25 \\ 151-175 & \$ 8.00 \\ 176-200 & \$ 9.00 \\ 201-225 & \$ 9.25 \\ 226-250 & \$ 9.50 \\ 251-275 & \$ 10.75 \\ 276-300 & \$ 11.00\end{array}$


PNL -3538

33679000545345

UC-41

CURRENT PERSONNEL DOSIMETRY PRACTICES

AT DOE FACILITIES

J. J. Fix, PNL Principal Investigator

J. M. Selby, PNL Project Manager

E. J. Vallario, DOE Project Manager(a)

May 1981

Prepared for

the U.S. Department of Energy

under Contract DE-AC06-76RLO 1830

Pacific Northwest Laboratory

Richland, Washington 99352

(a) Assistant Chief/Manager, Health Physics Programs

Division of Operational and

Environmental Safety

U.S. Department of Energy

Washington, DC 20545 


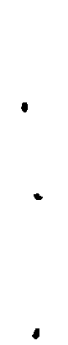




\section{FOREWORD}

The Operational and Environmental Safety (OES) Division of the Department of Energy (DOE) initiated a study during FY-80 to review the status of the DOE Radiation Records Repository following a preliminary analysis by OES which indicated a need to upgrade the repository.

The repository provides an overview of DOE and DOE contractor employee occupational radiation exposure, an assessment of transient worker exposure between DOE contractors, and information for the DOE annual report of occupational radiation exposure received by DOE and DOE contractor employees. This information is used to monitor DOE experience regarding cumulative employee and individual exposures as we 11 as identification of any trends.

The purpose of the study is to determine the adequacy of the present system, identify necessary short-term improvements and to propose feasible alternatives for an improved system. The study will include reports as follows:

- Current Personne1 Dosimetry Practices at DOE Facilities

- Overview of DOE Radiation Exposure Information Reporting System (REIRS)

- Alternatives to Provide Upgraded Occupational Exposure Record System.

This report constitutes the first topical report and is based on the responses provided by DOE contractors throughout the U.S. including all major contractors. Knowledge of the site specific records systems is a necessary beginning to identify feasible alternatives for upgrading the current occupational exposure repository.

E. J. Vallario

Assistant Chief/Manager

Health Physics Programs

Division of Operational and

Environmental Safety

U.S. Department of Energy 


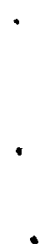


SUMMARY

The information presented in the following sections and in the appendices illustrates the diversity of personnel dosimetry practices at different DOE facilities. In several cases, unique dosimeter designs are in use at specific facilities generally involving unique methods of dose determination. Facilities using commercial dosimeters or dosimeters from other DOE facilities often were totally unaware of the specifics of the dosimeter design or dose assessment. Of the twenty-one facilities in which they or the commercial processor participated in the University of Michigan intercomparison study, only three facilities submitted their results as requested.

Only three parameters were included in the personnel occupational exposure records by all facilities. These are employee name, social security number, and whole body dose. Approximate percentages of some other parameters included in the record systems are sex (50\%), birthdate (90\%), occupation (26\%), previous employer radiation exposure (74\%), etc. Statistical analysis of the data for such parameters as sex versus dose distribution, age versus dose distribution, cumulative lifetime dose, etc. was apparently seldom done.

Less than $50 \%$ of the facilities reported having formal documentation for either the dosimeter, records system, or reader. Slightly greater than 50\% of facilities reported having routine procedures in place. These are considered maximum percentages because some respondents considered computer codes as formal documentation.

The repository receives data from DOE facilities regarding the
a) distribution of annual whole body doses,
b) significant internal depositions, and
c) individual doses upon termination.

It is expected that numerous differences exist in the dose data submitted by the different facilities. Areas of significant differences would likely include the determination of non-measurable doses, the methods used to determine previous employer radiation dose, the methods of determining cumulative 
radiation dose, and assessment of internal doses. Undoubtediy, the accuracy of the different dosimetry systems, especialily at low doses, is very important to the credibility of data summaries (e.g., man-rem) provided by the repository. 


\section{CONTENTS}

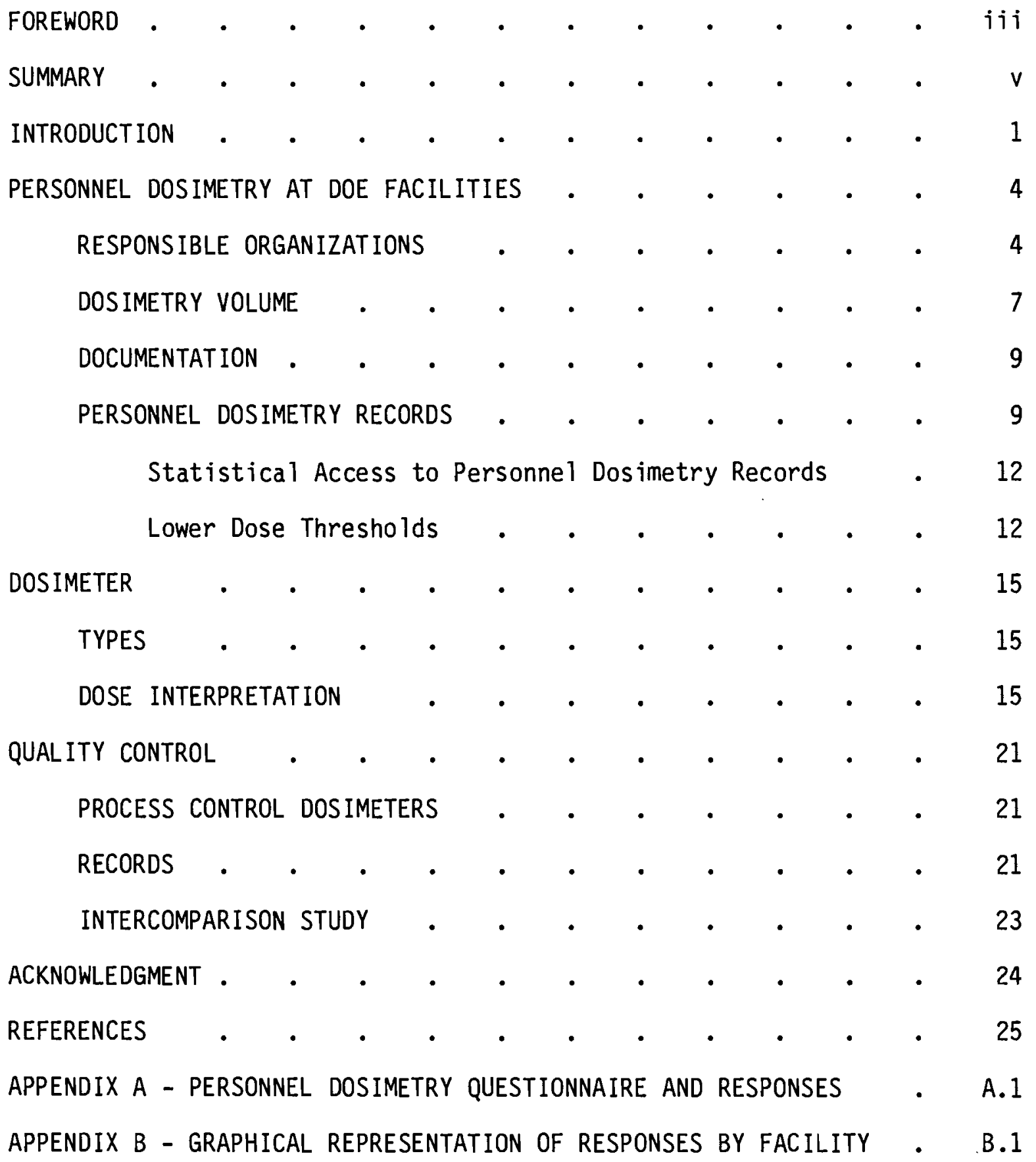




\section{FIGURES}

1 Personne 1 Dos imetry Documentation . . . . . . 10

2 Parameters Included Routinely in Personnel Dosimetry Record Systems

3 Percentage of Responses Reporting Capability to Statistically Access Personnel Records for Indicated Parameters .

4 Percentage of Facilities Which Have Identified Lower Dose Thresholds

5 Percentage of Facilities Answering Yes . . . . . 22

\section{TABLES}

1 DOE Facilities Included in Study . . . . . . 2

2 Organizations Responsible for Dosimetry Systems . . . 5

3 Current Dosimetry Workload and Historical Record Volume - 8

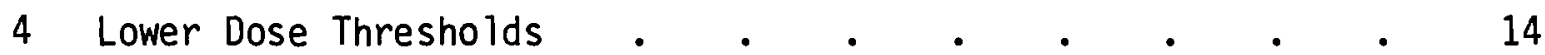

5 Dose Assessment and Dosimeter Filtration for DOE Facilities . 17 


\section{CURRENT PERSONNEL DOSIMETRY PRACTICES}

AT DOE FACILITIES $(a)$

\section{INTRODUCTION}

A study of the DOE personnel dosimetry records repository at Idaho Falls, Idaho and the relationship of these records with the individual records maintained by each of the DOE facilities was initiated during January 1980 . A questionnaire, included as Appendix $A$, was used to solicit information from each of the DOE facilities. Responses were received from many different facilities including several universities and all DOE national laboratories. A list of all facilities included in the study is shown in Table 1. Personnel dosimetry needs vary greatly between these facilities according to the type of operation, potential for radiation exposure, and number of personnel involved. Many of the DOE national laboratories have designed their personnel dosimetry system to be particularly sensitive to their operations. Several facilities, especially smaller operations, utilize personnel dosimetry services provided by commerical vendors.

The information provided by the facilities has been summarized in the body of the report; however. Appendix $B$ contains information illustrative of the input by each facility. Specific areas covered in the questionnaire include:

- Who designed the dosimeter?

- Who provides routine dosimeter fabrication, readout, and record keeping?

- What parameters are included in the record system?

- What are the lower dose thresholds and is an environmental contribution subtracted?

(a) DOE facilities refers to facilities either government or privately owned conducting DOE programs. 
TABLE 1. DOE Facilities Included in Study

DOE Facility

Ames Laboratory

Argonne National Laboratory (ANL)

Bartlesville Energy Technology Center (BETC)

Bates Linear Accelerator

Brookhaven National Laboratory (BNL)

Environmental Measurements Laboratory

Fermi Laboratory

Goodyear Atomic Corporation

Hanford Plant

Idaho National Engineering Laboratory (INEL)

Inhalation Toxicology Research Institute

Kansas State University

Laramie Energy Technology Center

Lawrence Berkeley Laboratory (LBL)

Lawrence Livermore Laboratory (LLL)

Los Alamos Scientific Laboratory (LASL)

Mound Laboratory

National Lead Company of Ohio

National Magnet Laboratory

Nevada Testing Site (NTS)

Oak Ridge, Associated Universities

Oak Ridge National Laboratory (ORNL)

Ohio State University

Paducah Gaseous Diffusion Plant

Pantex Plant

Pinellas Plant

Princeton University

Purdue University

Rockwell International

Rocky Flats Plant

Sandia National Laboratories-Albuquerque -Livermore

Savannah River Plant (SRP)

Stanford Linear Accelerator (SLAC)

University of California-Davis

University of California

Laboratory of Radiobiology

University of $\mathrm{Cal}$ ifornia

Laboratory of Nuclear Medicine

University of Colorado

University of Notre Dame

University of Pittsburgh

University of Tennessee

Comparative Animal Research Laboratory

University of Utah

Radiobiology Laboratory

Yale University
Location

Ames, Iowa

Argonne, Illinois

Bartlesville, Ok lahoma

Middleton, Massachusetts

Upton, New York

New York, New York

Batavia, Illinois

Piketon, Ohio

Richland, Washington

Idaho Fails, Idaho

Albuquerque, New Mexico

Manhattan, Kansas

Laramie, Wyoming

Berkeley, California

Livermore, California

Mercury, Nevada

Los Alamos, New Mexico

Miamisberg, Ohio

Cincinnati, Ohio

Cambridge, Massachustts

Las Vegas, Nevada

Oak Ridge, Tennessee

Oak Ridge, Tennessee

Columbus, Ohio

Paducah, Kentucky

Amarillo, Texas

St. Petersburg, Florida

Princeton, New Jersey

West Lafayette, Indiana

Canoga Park, California

Golden, Colorado

Albuquerque, New Mexico

Livermore, California

Aiken, South Carolina

Stanford, California

Davis, California

San Francisco, California

Los Angeles, California

Boulder, Colorado

Notre Dame, Indiana

Pittsburgh, Pennsylvania

Oak Ridge, Tennessee

Salt Lake, Utah

New Haven, Connecticut 
- Volume of dosimeter records both current and historical.

- Status of formal and procedure documentation.

- Definition of absorbed dose depth in tissue.

- Description of several personnel dosimetry practices. 


\section{PERSONNEL DOSIMETRY AT DOE FACILITIES}

\section{RESPONSIBLE ORGANIZATIONS}

Organizations responsible for components of the routine personnel dosimetry service are shown in Table 2. The facilities were identified according to one of the following:

$\begin{array}{ll}\text { DOE Laboratory } & \text { (DL) } \\ \text { Contractor Laboratory } & \text { (CL) } \\ \text { University } & \text { (U) } \\ \text { Energy Center } & \text { (EC) } \\ \text { Private Contractor } & \text { (PC) }\end{array}$

The dosimetry volume classifications for the number of dosimeters processed per year are as follows:

$\begin{array}{ll}\text { Large } & >10,000 \\ \text { Medium } & 1,000-10,000 \\ \text { Sma11 } & <1,000\end{array}$

Components identified were original dosimeter design, type of dosimeter, routine dosimeter fabrication, dosimeter readout, and personnel records. Services provided by commercial vendors were identified.

It is apparent from Table 2 that the majority of DOE laboratories, which generally involve large dosimetry volumes, utilize dosimeter systems which were designed in-house. The majority of DOE laboratories provide all aspects of the routine dosimetry service including routine dosimeter fabrication, readout, and personnel records. All other facilities generally contract commercial venders to provide the routine personnel dosimetry program with the exception of personnel records. In a few cases, personnel records were maintained by both the DOE facility and the commercial vender. 


\section{TABLE 2. Organizations Responsible for Dosimetry Systems (a)}

\begin{tabular}{|c|c|c|c|c|c|c|c|}
\hline \multirow[b]{2}{*}{ Facility } & \multirow[b]{2}{*}{$\begin{array}{l}\text { Facility } \\
\text { Type } \\
\end{array}$} & \multirow[b]{2}{*}{$\begin{array}{c}\text { Dosimeter } \\
\text { Volume }\end{array}$} & \multirow[b]{2}{*}{$\begin{array}{l}\text { Dosimeter } \\
\text { Design }\end{array}$} & \multirow[b]{2}{*}{$\begin{array}{l}\text { Dosimeter } \\
\text { Type }\end{array}$} & \multicolumn{3}{|c|}{ Rout ine Program } \\
\hline & & & & & $\begin{array}{l}\text { Dosimeter } \\
\text { Fabrication } \\
\end{array}$ & $\begin{array}{l}\text { Dosimeter } \\
\text { Readout }\end{array}$ & $\begin{array}{r}\text { Personnel } \\
\text { Records } \\
\end{array}$ \\
\hline A & $\mathrm{DL}$ & Large & Landauer & Film & Landauer & Landauer & Landauer/IH \\
\hline B & $\mathrm{DL}$ & Medium & IH & Film & IH & IH & IH \\
\hline $\mathrm{c}$ & $\mathrm{DL}$ & Medium & IH & TLD & IH & IH & IH \\
\hline D & DL & Large & IH & TLD & IH & IH & IH \\
\hline $\mathrm{E}$ & $\mathrm{DL}$ & Large & IH & TLD/Film & IH & IH & IH \\
\hline $\bar{F}$ & $\mathrm{DL}$ & Medium & IH & TLD & IH & IH & IH \\
\hline G & $\mathrm{DL}$ & Large & IH & TLD & IH & IH & IH \\
\hline$H(b)$ & $\mathrm{DL}$ & Medium & Landquer & $\mathrm{Film}$ & Landauer & Landauer & IH \\
\hline & & & $\mathrm{IH}-\mathrm{D}(\mathrm{C})$ & TLD & IH-D & IH-D & IH \\
\hline I & $\mathrm{DL}$ & Large & IH & TLD & IH & IH & IH \\
\hline$j$ & $\mathrm{DL}$ & Large & IH & TLD & IH & IH & IH \\
\hline K & $\mathrm{DL}$ & Large & $I H / I H-E(d)$ & TLD/Film & IH & IH & IH \\
\hline$L(e)$ & $\mathrm{DL}$ & Large & IH & Film & IH & IH & IH \\
\hline & & & Harshaw & TLD & Harshaw & IH & IH \\
\hline M & $\mathrm{DL}$ & Large & IH/Harshaw & TLD & IH & IH & IH \\
\hline $\mathrm{N}$ & $\mathrm{DL}$ & Medium & $\mathrm{IH} / \mathrm{IH}-\mathrm{I}$ & TLD & IH-P & IH-P & IH-P \\
\hline 0 & $\mathrm{DL}$ & Large & IH & TLD & IH & IH & IH \\
\hline $\mathrm{P}$ & $\mathrm{DL}$ & Large & IH & TLD & IH & IH & IH \\
\hline 0 & $\mathrm{DL}$ & Large & IH & TLD & IH & IH & $\mathrm{IH}$ \\
\hline $\mathrm{R}$ & DL & Large & $I H-Q / P(g)$ & TLD & IH & IH & IH \\
\hline & $\mathrm{DL}$ & Large & IH & TLD & IH & IH & IH \\
\hline$T(e)$ & $\mathrm{CL}$ & Medium & $I H-E$ & $\mathrm{Film}$ & IH & IH & IH \\
\hline & & & Harshaw & TLD & IH & $\mathrm{IH}$ & $\mathrm{IH}$ \\
\hline U & EC & Small & ICN & Film & ICN & ICN & ICN \\
\hline v & EC & Small & Landauer & $\mathrm{Film}$ & Landauer & Landauer & IH \\
\hline$W$ & PC & Medium & $\mathrm{IH}-\mathrm{E}$ & TLD/Film & IH & IH & IH \\
\hline$x$ & $\mathrm{CL}$ & Medium & Eberline & TLD & Eberline & Eberline & Eberline \\
\hline Y & PC & Medium & Landauer & $\mathrm{Film}$ & Landauer & Landauer & IH \\
\hline z & U & Med i um & Landauer & Film & Landauer & Landauer & Landauer \\
\hline AA & $\mathrm{U}$ & Sma 11 & Landauer & Film & Landauer & Landauer & Landauer \\
\hline $\mathrm{BB}$ & U & Medium & Landauer & TLD & Landauer & Landauer & IH \\
\hline CC & U & Sma 11 & Eberline & TLD & Eberline & Eberline & IH \\
\hline $\mathrm{DD}$ & U & Large & Landauer & $\mathrm{Film}$ & Landauer & Landauer & Landauer/IH \\
\hline $\mathrm{EE}$ & U & Medium & RDC & Film & RDC & RDC & RDC \\
\hline $\mathrm{FF}$ & U & Medium & Landauer & $\mathrm{Film}$ & Landauer & Landauer & Landauer/IH \\
\hline GG & U & Medium & RDC & TLD/Film & RDC & RDC & CRSO \\
\hline $\mathrm{HH}$ & $\mathrm{CL}$ & Sma 11 & RDC & TLD/Film & RDC & RDC & $\mathrm{RDC} / \mathrm{IH}$ \\
\hline II & $\mathrm{CL}$ & Sma 11 & - & Film & CRSO & CRSO & CRSO \\
\hline jJ & U & Small & Landauer & $\mathrm{Film}$ & Landauer & Landauer & Landauer \\
\hline KK & U & Sma 11 & UST & Film & UST & UST & IH \\
\hline LL & U & Medium & Eberline & TLD & IH & IH & IH \\
\hline MM & $\mathrm{CL}$ & Medium & IH & TLD & IH & IH & IH \\
\hline NN & $C L$ & Medium & Landauer & Film & Landauer & Landauer & Landauer \\
\hline 00 & U & Medium & RDC & TLD/Film & RDC & RDC & TDC/IH \\
\hline $\mathrm{PP}$ & $\mathrm{CL}$ & Sma 11 & Eberline & TLD & IH & IH & IH \\
\hline$Q Q$ & $\mathrm{DL}$ & Small & Landauer & TLD/Film & Landauer & Landauer & IH \\
\hline
\end{tabular}

(a) $I H=$ In House, RDC = Radiation Detection Company, CRSO = Campus Radiation Safety Office,
ICN = ICN Pharmaceuticals.

(b) Facility $\mathrm{H}$ has two routine dosimeters for different programs; one dosimeter provided by Landauer, the other from DOE facility D.

(c) Dosimeter design, dosimeter fabrication, and dosimeter readout provided by DOE facility $D$.

(d) Dosimeter design modified from facility E design.

(e) Facility currently changing dosimeter system from Film to TLD.

(f) Dosimeter design of facility $N$ based on design of facility $I$.

(g) Dosimeter design based on concepts of facilities $Q$ and $P$. 
The data in Table 2 indicates the following usage among the 43 DOE facilities regarding the type of personnel dosimeter routinely used:

$\begin{array}{lr}\text { TLD } & 19(21) \\ \text { Film } & 16(14) \\ \text { TLD/Film } & 8(8)\end{array}$

Two of the facilities ( $L$ and $T$ ) are currently in the process of converting from in-house designed film dosimeters to commercially designed TL dosimeters. The numbers in parenthesis show the change in the usage following adoption of the new dosimeters.

Responsibility for routine dosimeter fabrication is shown in Table 2 and is summarized according to the type of facility as follows:

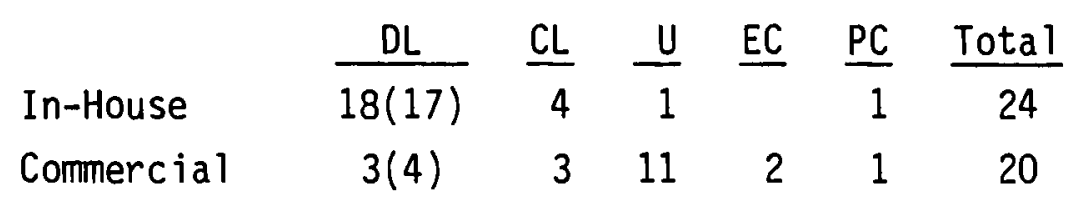

The "In-House" category listed for DOE laboratories (DL) includes two laboratories ( $H$ and $N$ ) which receive services from other DOE laboratories. Also, one facility $(L)$ is presently changing their system from in-house to commercial and this change is shown by the parenthesis. Also, a contractor laboratory (facility II) associated with a university receives service from the University Radiation Safety Office. This has been classified as in-house. The total is 44 because facility $\mathrm{H}$ receives two dosimetry services; one in-house and the other commercial.

Dosimeter readout for the facilities in Table 2 is summarized as follows:

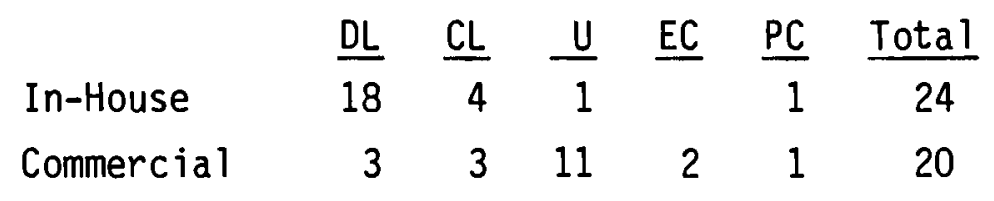

This summary is identical to the previous summary for dosimeter fabrication. The only difference is that facility $L$ will provide in-house dosimeter readout for their new system as they have been doing for the old system. 
Personnel records are sumarized as follows:

\begin{tabular}{|c|c|c|c|c|c|c|}
\hline & $\underline{D L}$ & $\mathrm{CL}$ & $U$ & $\underline{E C}$ & $\underline{P C}$ & Total \\
\hline n-House & 21 & 5 & 8 & 1 & 2 & 37 \\
\hline ommercial & (1) & $2(3)$ & $4(7)$ & 1 & & 7 \\
\hline
\end{tabular}

The number of facilities which have both in-house and commercial personne 1 record systems are indicated by the parenthesis. For example, one DOE laboratory (A), one contractor laboratory $(H H)$ and three universities (DD, FF, and 00 ) have an in-house program and a commercial service.

It is apparent that most facilities maintain their own personnel records. of the seven facilities which rely on a commercial service, 3 facilities have a small and 4 have medium annual dosimeter volumes.

\section{DOSIMETRY VOLUME}

The DOE facilities included in this study are very diverse in the type and size of DOE programs conducted. Table 3 shows the dosimetry work load reported by those facilities during 1979 as well as an estimate of the historical record volume in terms of man-years of record data. During 1979, these facilities processed approximately 760,943 dosimeters for about 175,852 people. The number of dosimeters processed is generally much greater than the number of people on account of frequent dosimeter exchanges (monthly or quarterly) for part of the personnel. For example, 3000 employees receiving monthly exchanges would account for 36,000 dosimeters processed per year (e.g., 3000/month $\times 12$ months/year).

In addition to the personnel dosimetry records, many facilities have numerous supporting records such as radiation incident reports, radiation work permits, monitoring survey results, laboratory data, etc. These records support the data in the personnel dosimetry files. 
TABLE 3. Current Dosimetry Workload and Historical Record Volume

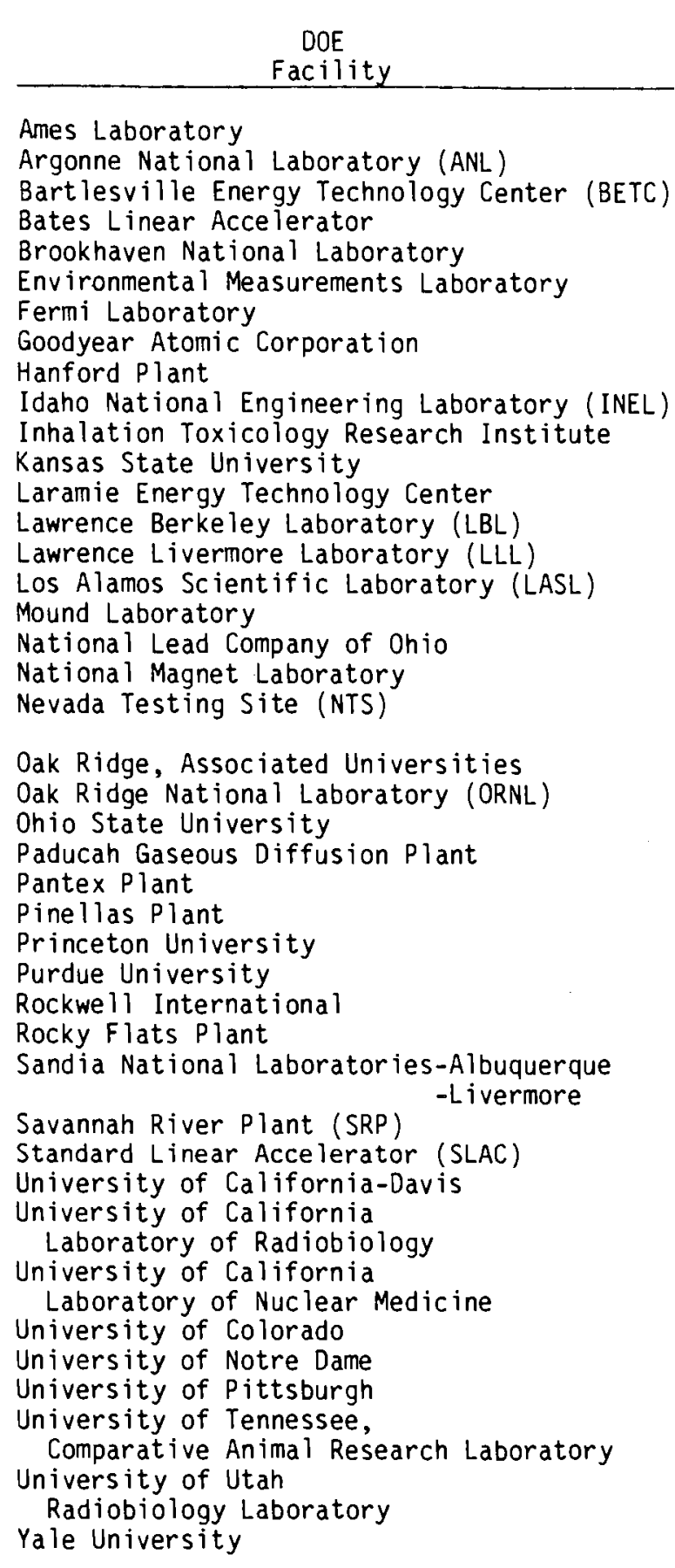

\begin{tabular}{|c|c|c|}
\hline \multicolumn{2}{|c|}{1979} & \multirow{2}{*}{$\begin{array}{l}\text { Approximate } \\
\text { Man-Years of } \\
\text { Record Data } \\
\end{array}$} \\
\hline $\begin{array}{r}\text { Dosimeters } \\
\text { Processed } \\
\end{array}$ & $\begin{array}{c}\text { Number of } \\
\text { People } \\
\end{array}$ & \\
\hline 892 & 91 & $2,100(a)$ \\
\hline 30,000 & 2,500 & $(37)(b)$ \\
\hline 25 & 12 & 200 \\
\hline 5,000 & $1,700(c)$ & 2,000 \\
\hline 43,110 & 2,437 & 70,000 \\
\hline 500 & 35 & 1,500 \\
\hline 31,000 & 4,150 & 330,000 \\
\hline 5,000 & 1,000 & 50,000 \\
\hline 82,000 & 28,000 & 350,000 \\
\hline 100,000 & 41,500 & 180,000 \\
\hline 2,000 & 250 & 2,000 \\
\hline 2,400 & 200 & 3,000 \\
\hline 192 & 16 & 75 \\
\hline 4,500 & 4,000 & 70,000 \\
\hline 40,000 & 23,000 & 400,000 \\
\hline 79,000 & 7,700 & 123,000 \\
\hline 14,000 & 1,450 & $(1947-1979)(d)$ \\
\hline 9,230 & 1,295 & 40,000 \\
\hline 1,000 & 90 & 300 \\
\hline 81,711 & 19,267 & $1,066,127$ \\
\hline 800 & 500 & (total records) \\
\hline 31,500 & 8,500 & 200,000 \\
\hline 96 & 8 & $(35)(b)$ \\
\hline 10,000 & 2,500 & 37,000 \\
\hline 4,400 & 700 & 20,000 \\
\hline 2,250 & 200 & 4,400 \\
\hline 16,000 & 1,800 & - \\
\hline 5,000 & 320 & 10,000 \\
\hline 6,000 & $350(a)$ & 8,050 \\
\hline 45,000 & $3,400(a)$ & 100,000 \\
\hline 16,000 & 4,700 & 60,000 \\
\hline 1,285 & 1,075 & $(15)(b)$ \\
\hline 68,528 & 7,605 & 200,000 \\
\hline 3,000 & 2,200 & 20,000 \\
\hline 1,460 & 135 & 1,600 \\
\hline 240 & 20 & 400 \\
\hline 544 & 145 & 2,000 \\
\hline 3,400 & 350 & 2,500 \\
\hline 391 & 51 & 375 \\
\hline 9,800 & 1.300 & - \\
\hline 450 & 115 & 3,000 \\
\hline 1,200 & 100 & 2,000 \\
\hline 2,040 & 85 & 7,000 \\
\hline
\end{tabular}

(a) Not including visitors and other contractors.

(b) Years of data.

(c) 200 permanent, 2000 visitor.

(d) Inclusive years of data. 
DOCUMENTATION

There appeared to be much latitude between facilities regarding documentation of the dosimetry system and personnel exposure records. Results of Questions \#1 through \#4 of Section I of the Questionnarie (see Appendix A) are summarized in Figure 1 . It is apparent that several facilities have not documented the ir system. Confirmatory answers (Yes in Figure 1) provided by several facilities for formal documentation in Questions 1 through 3 involved reference to in-house procedures, descriptions of computer codes, etc., and are not consistent with the formal laboratory reports or publication in the open literature reported by other facilities.

Preferably, a 1) formal document in which the fundamental theory and system design is presented and 2) routine procedures would be available for each major component of the system. The formal document should be available for public distribution and the procedures simply implement the system. Several facilities receiving a commercial service simply reference the vender information. In some cases, facilities receiving a commercial service knew very little about the dosimetry system or documentation.

\title{
PERSONNEL DOSIMETRY RECORDS
}

Information routinely included in the personnel dosimetry records maintained by the various DOE facilities is shown in Figure 2. Three parameters were recorded by everyone as follows:

\author{
Name \\ Social Security Number \\ Whole Body Dose
}

Several parameters of interest for statistical analysis of the data, such as sex or age (birth date), are not recorded by everyone. Only one-half, approximately, of the facilities reported including sex in the records system. 


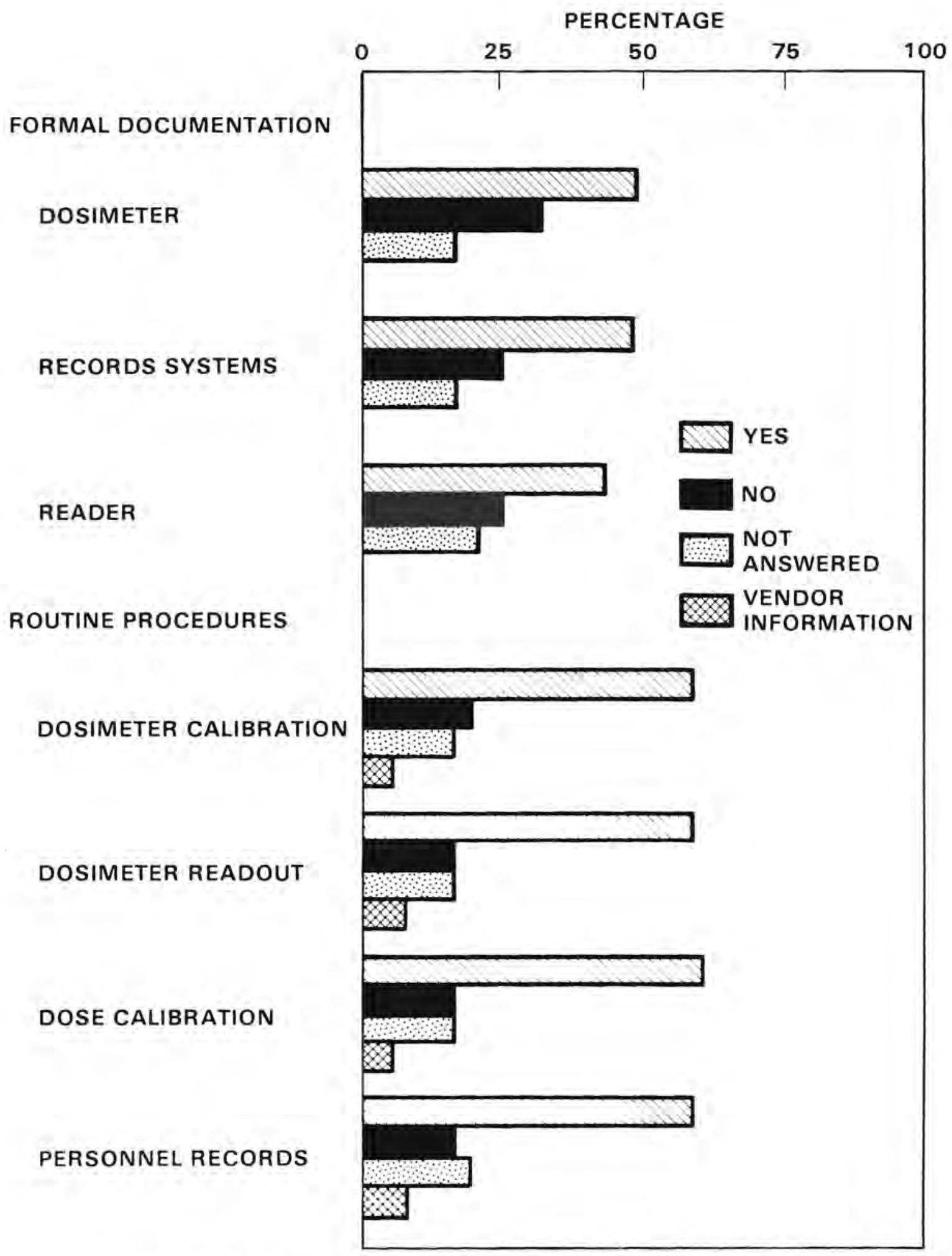

FIGURE 1. Personnel Dosimetry Documentation 
NAME

BIRTHDATE

SEX

SOCIAL SECURITY \#

PAYROLL \#

OCCUPATION

HIRE DATE

FACILITY TYPE

PREVIOUS EXP.

SYSTEMIC BURDEN

PERCENT OF MPPB

PERCENT OF ORGAN BURDEN

INTAKE DATE OF DEPOSITION

ORGAN OF DEPOSITION

RADIONUCLIDE OF DEPOSITION

ANNUAL DOSE FROM DEPOSITION

METHOD OF ANALYSIS

NONPENETRATING DOSE DATA

PENETRATING DOSE DATA

NEUTRON TOTAL DOSE DATA

SLOW NEUTRON DOSE DATA

FAST NEUTRON DOSE DATA

HIGH LET

WHOLE BODY DOSE

SKIN DOSE

EXTREMITY DOSE

DOSIMETER EXCHANGE FREQUENCY

WORKER

JOB ASSIGNMENT

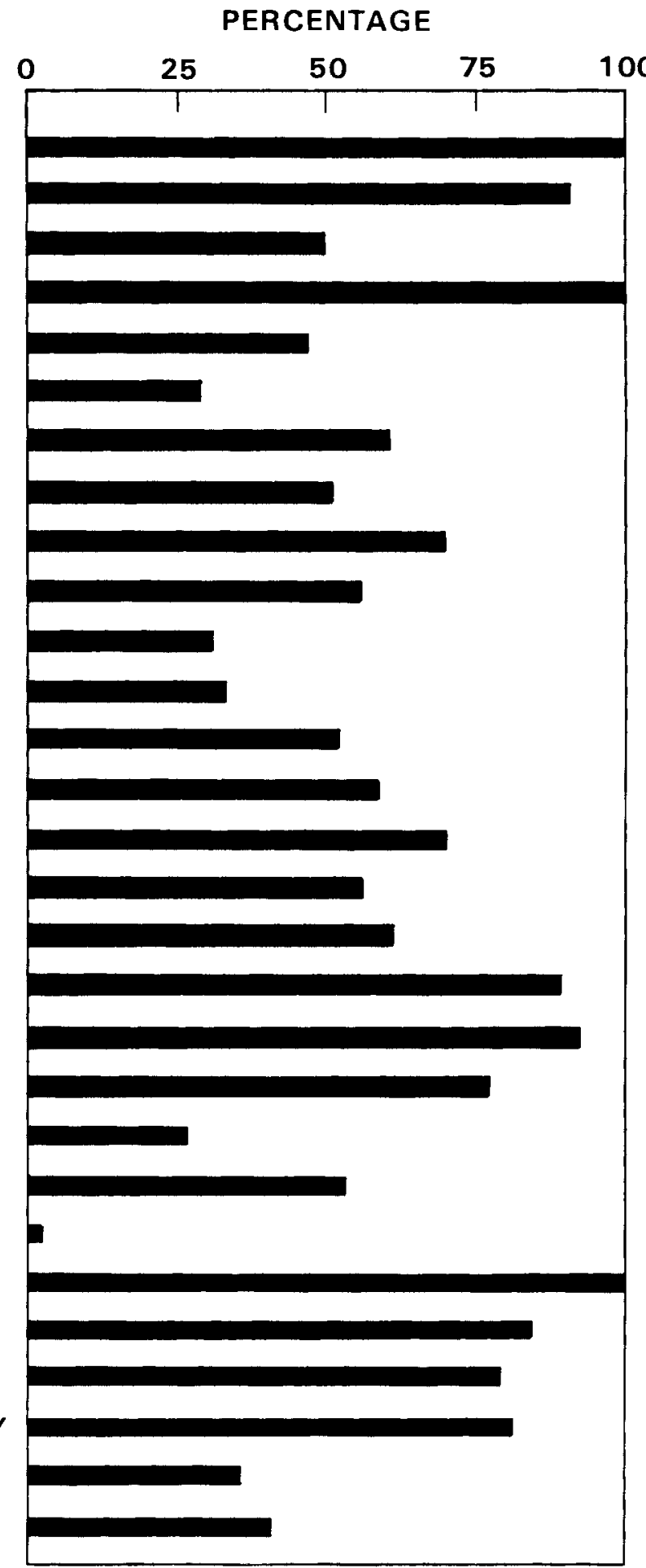

FIGURE 2. Parameters Included Routinely in Personnel Dosimetry Record Systems 
Likely, there was some confusion in answering questions $7 P$ on dosimeter data and $7 Q$ on dosimeter dose (see Questionnaire, Appendix A). The questions were asked to determine how many facilities maintain records of dosimeter data such as TLD chip readings, film densities, etc., in addition to the interpreted dose. It is apparent from the comments on the questionnaires that several respondents understood this and answered accordingly. However some respondents seemed perplexed in determining the difference between dosimeter dose and dosimeter data.

\section{Statistical Access to Personnel Dosimetry Records}

As expected, few facilities have the existing capability to examine the personnel records system in terms of the distribution of sex versus dose, age versus dose, and sex and age versus dose as shown in Figure 3. Generally, facilities have the existing capability of monitoring individual employee dose for the current year. They apparently also classify their employees according to facility type as required on DOE Form 190 for input to the repository. However, for some facilities, the entire facility is simply one classification (i.e., general research, accelerator, etc.). In these cases, the facility must simply distinguish between visitor exposure and employee exposure.

Few facilities monitor the cumulative dose for their employees as distinct from the annual dose. Some facilities (Appendix $A$ ) indicated the ability to monitor lifetime employee dose for employees while at their facilities. Generally, employee exposure received while at a previous employer would not be included.

\section{Lower Dose Thresholds}

Question \#13 of the Questionnaire asked whether or not a lower dose threshold was used below which a 11 doses were considered background (i.e., zero). Figure 4 illustrates the percentage of respondents indicating that they routinely employ lower dose thresholds for each radiation category. Table 4 shows the actual dose thresholds reported. From the data, it is apparent that most facilities regard 10 mRem as the lower dose threshold. 


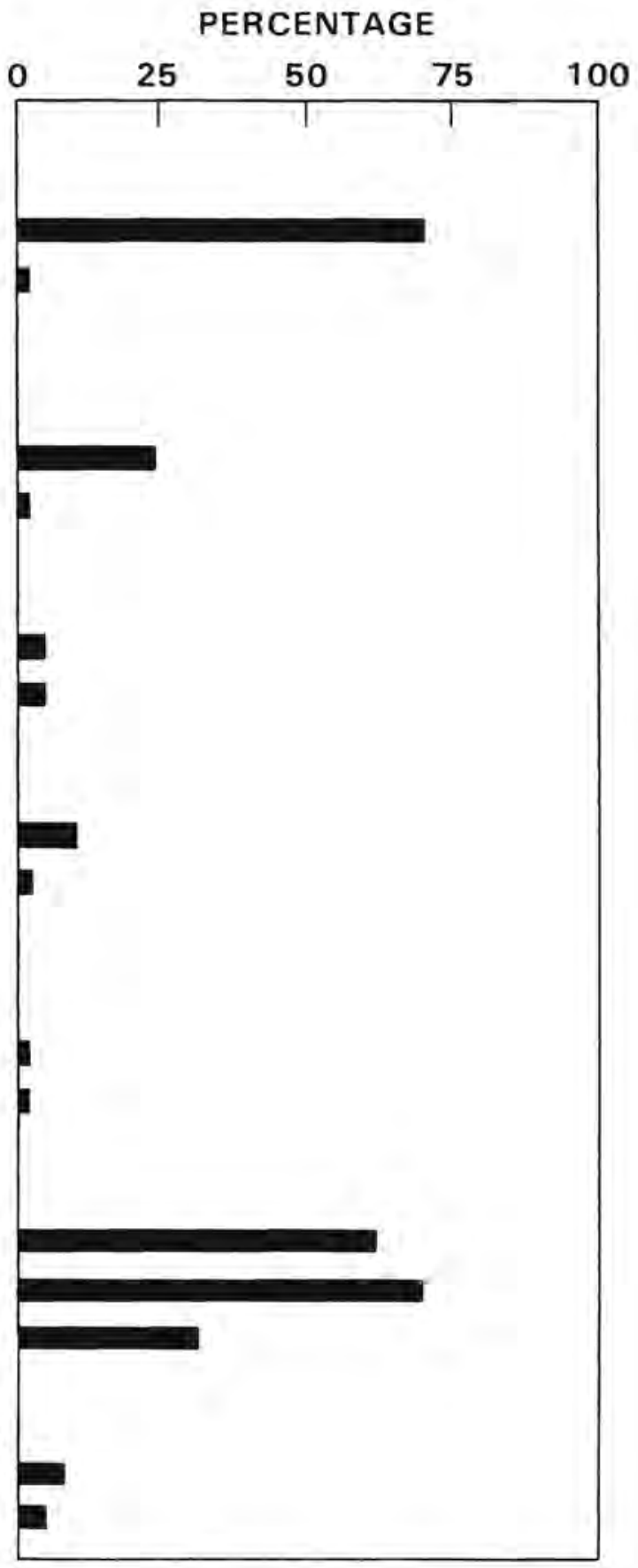

FIGURE 3. Percentage of Responses Reporting Capability to Statistically Access Personnel Records for Indicated Parameters 


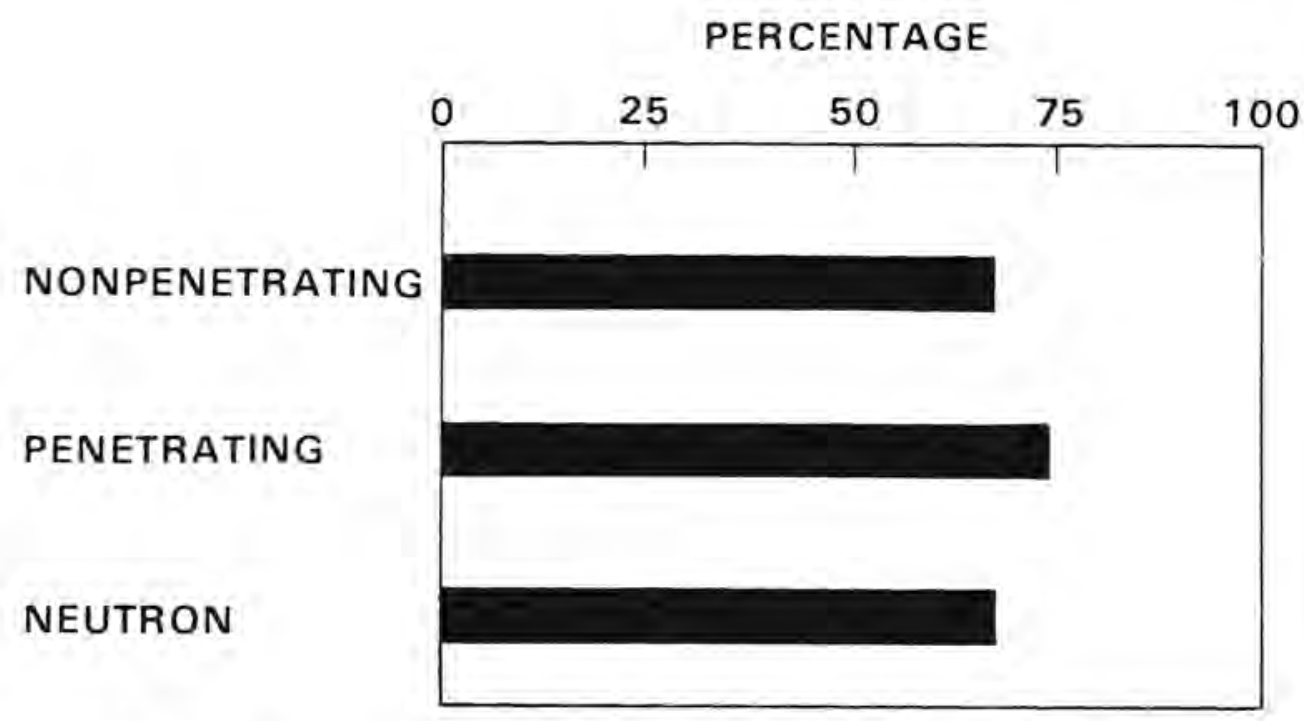

FIGURE 4. Percentage of Facilities Which Have Identified Lower Dose Thresholds

TABLE 4. Lower Dose Thresholds (a)

\begin{tabular}{|c|c|c|c|c|c|}
\hline \multirow[b]{2}{*}{ Dose (mrem) } & \multirow[b]{2}{*}{ Nonpenetrating } & \multirow[b]{2}{*}{ Penetrating } & \multicolumn{3}{|c|}{ Neutron } \\
\hline & & & Thermal & Fast & Total \\
\hline 5 & 5 & 5 & & & 3 \\
\hline 10 & 13 & 17 & 6 & 1 & 8 \\
\hline 15 & & 2 & & & 1 \\
\hline 20 & $5^{(b)}$ & $5^{(b)}$ & & 4 & 5 \\
\hline 30 & & $3^{(c)}$ & 1 & 1 & 2 \\
\hline 40 & $3^{(c)}$ & & & & 1 \\
\hline 50 & & & & & 3 \\
\hline 50 & & & & & \\
\hline
\end{tabular}

(a) Total responses in each category were 29 nonpenetrating, 32 penetrating and 29 neutron.

(b) One facility reported $20 \mathrm{mRad}+1.6 \mathrm{mRad} / \mathrm{wk}$.

(c) One facility reported $30 \mathrm{mRad} / \mathrm{fi} \mathrm{m}$ nonpenetrating.

(d) One facility reported $120 \mathrm{mrem}$ at $14 \mathrm{MeV}$. 


\section{DOSIMETER}

\section{TYPES}

As shown in Table 2, 16 of the facilities rely on film dosimetry exclusively and 8 other facilities utilize film in conjunction with TL dosimeters. The other 19 facilities utilize TLDs. However, 2 of the 16 facilities using film currently are changing to TL dosimeters.

\section{DOSE INTERPRETATION}

Table 5 summarizes the responses to Questions 17, 18, and 19 of the questionnaire. The diversity of dose assessment methodology is apparent. Most facilities responded that they determine the nonpenetrating dose at a depth of $7 \mathrm{mg} / \mathrm{cm}^{2}$ and the penetrating dose at a depth of $1.0 \mathrm{~cm}$. However, the filtration on the dosimeter shown in columns 7, 8, 9, and 10 of Table 5 are generally greatly different from $7 \mathrm{mg} / \mathrm{cm}^{2}$ and $1000 \mathrm{mg} / \mathrm{cm}^{2}$ or being tissue equivalent. In these cases, the dose may be assessed at the stated depth if the field radiation spectrum and dosimeter laboratory calibration spectrum are similar and the dosimeter is calibrated to tissue dose at $7 \mathrm{mg} / \mathrm{cm}^{2}$ and $1000 \mathrm{mg} / \mathrm{cm}^{2}$.

Several facilities indicated that they were unaware of the depth of the dose assessment or the dosimeter filtration. Generally these facilities receive dosimetry services commercially or from another facility as shown in Table 2.

From the information in Table 5, a summary of the depths at which nonpenetrating dose is assessed follows:

$\begin{array}{lr}7 \mathrm{mg} / \mathrm{cm}^{2} & 18 \\ 10 \mathrm{mg} / \mathrm{cm}^{2} & 2 \\ 15 \mathrm{mg} / \mathrm{cm}^{2} & 1 \\ 23 \mathrm{mg} / \mathrm{cm}^{2} & 1 \\ 25 \mathrm{mg} / \mathrm{cm}^{2} & 3 \\ 32 \mathrm{mg} / \mathrm{cm}^{2} & 1 \\ 52 \mathrm{mg} / \mathrm{cm}^{2} & 1 \\ \text { Not answered } & 6 \\ \text { Unknown } & 9\end{array}$


Similarly the depths specified in Table 5 for penetrating dose are

$\begin{array}{lr}1 \mathrm{~cm} & 12 \\ 2 \mathrm{~cm} & 1 \\ 5 \mathrm{~cm} & 1 \\ 270 \mathrm{mg} / \mathrm{cm}^{2} & 1 \\ 285 \mathrm{mg} / \mathrm{cm}^{2} & 2 \\ 322 \mathrm{mg} / \mathrm{cm}^{2} & 1 \\ 1400 \mathrm{mg} / \mathrm{cm}^{2} & 1 \\ \text { Cd Ratio } & 1 \\ 0 \quad 1 \\ \text { Not answered } & 9 \\ \text { Unknown } & 14\end{array}$

Several facilities which either did not answer or answered unknown receive commercial services.

Dosimeter design and calibration techniques are important considerations in assessing depth dose in tissue. Facilities which have dosimeter designs different from $7 \mathrm{mg} / \mathrm{cm}^{2}$ tissue equivalent (nonpenetrating) and $1000 \mathrm{mg} / \mathrm{cm}^{2}$ tissue equivalent (penetrating) must determine their dosimeter response to sources calibrated to the desired tissue depth (i.e., 7 and $1000 \mathrm{mg} / \mathrm{cm}^{2}$ ). Also, the radiation type and spectrum in the field must be similar to the calibration spectrums. 
TABLE 5. Dose Assessment and Dosimeter Filtration for DOE Facilities

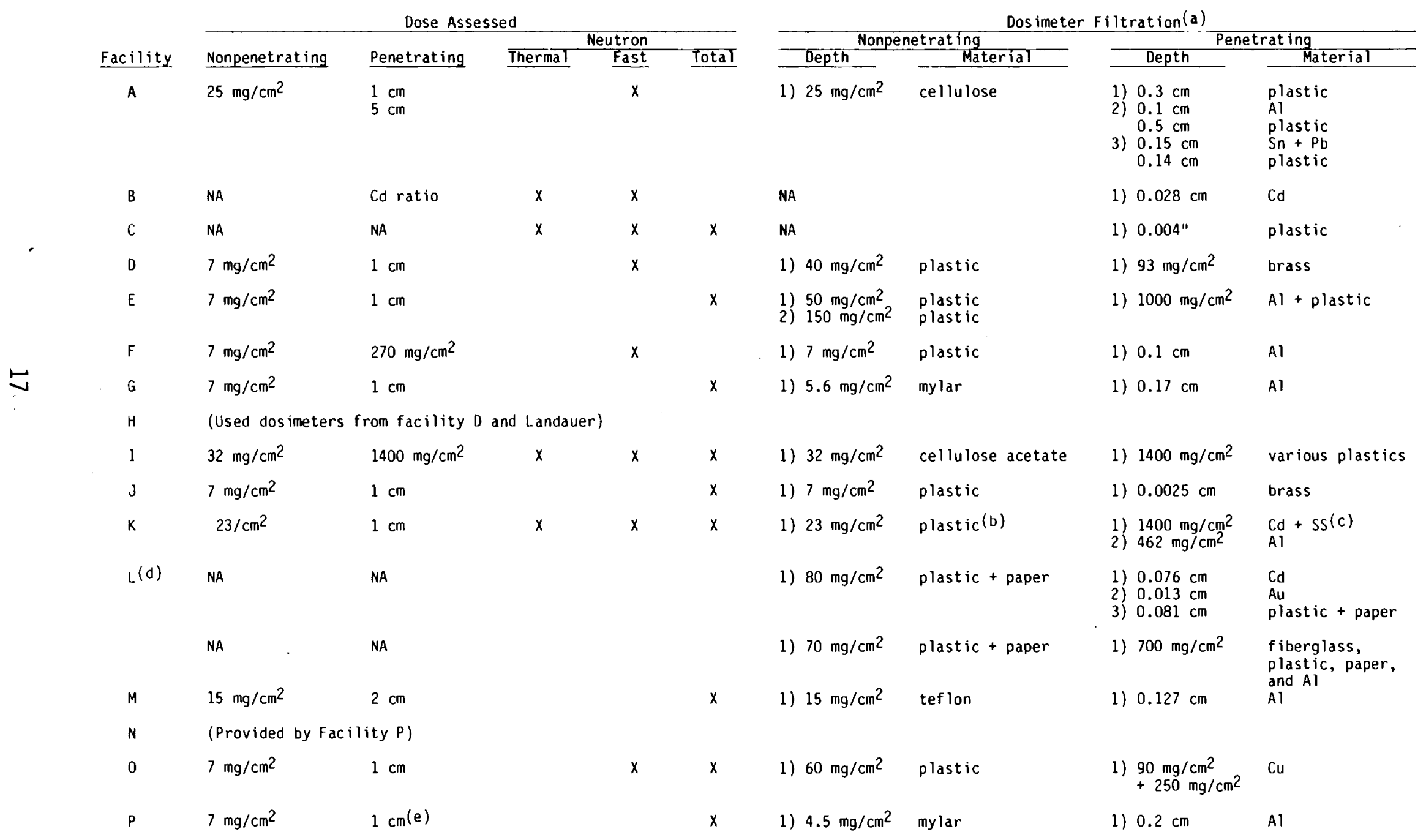


TABLE 5. (Contd)

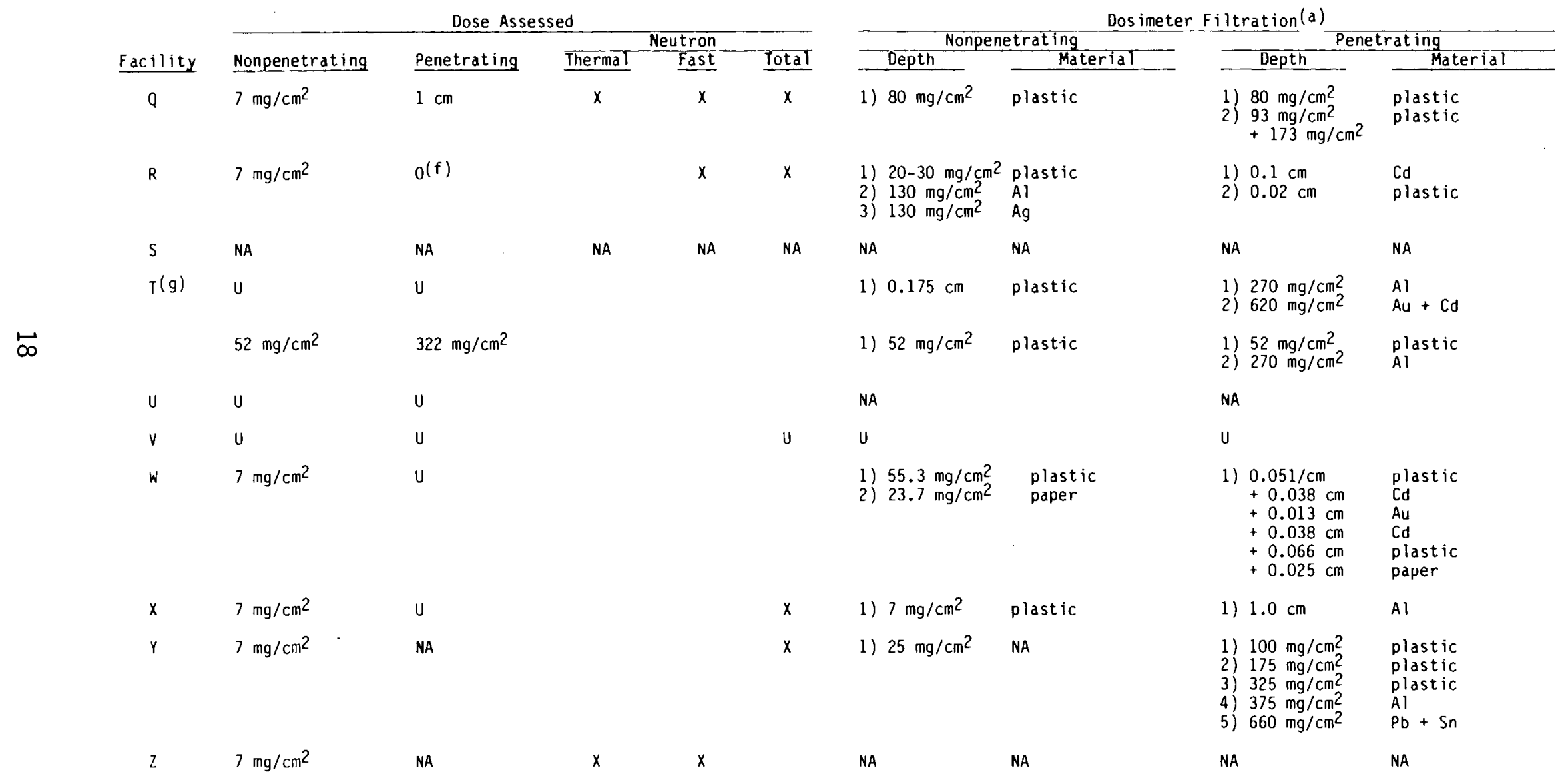




\section{TABLE 5. (Contd)}

\begin{tabular}{|c|c|c|c|c|c|c|c|c|c|}
\hline \multirow[b]{2}{*}{ Facility } & \multicolumn{5}{|c|}{ Dose Assessed } & \multicolumn{4}{|c|}{ Dosimeter Filtration (a) } \\
\hline & Nonpenetrating & Penetrating & Thermal & $\frac{\text { Neutron }}{\text { Fast }}$ & Total & Depth & $\frac{1 \text { etrating }}{\text { Material }}$ & Depth & $\frac{\text { rating }}{\text { Material }}$ \\
\hline AA & $25 \mathrm{mg} / \mathrm{cm}^{2}$ & u & & & & 1) $25 \mathrm{mg} / \mathrm{cm}^{2}$ & NA & $\begin{array}{l}\text { 1) } 100 \mathrm{mg} / \mathrm{cm}^{2} \\
175 \mathrm{mg} / \mathrm{cm}^{2} \\
\text { 3) } 325 \mathrm{mg} / \mathrm{cm}^{2} \\
\text { 4) } 375 \mathrm{mg} / \mathrm{cm}^{2} \\
\text { 5) NA }\end{array}$ & $\begin{array}{l}\text { plastic } \\
\text { plastic } \\
\text { plastic } \\
\text { Al } \\
60 \% \mathrm{~Pb}+40 \% \mathrm{Sn}\end{array}$ \\
\hline BB & $u(h)$ & $U(h)$ & $x$ & $x$ & & $N A(h)$ & $N A(h)$ & NA $(h)$ & $N A(h)$ \\
\hline CC & $10 \mathrm{mg} / \mathrm{cm}^{2}$ & $285 \mathrm{mg} / \mathrm{cm}^{2}$ & $x$ & $x$ & & 1) $10 \mathrm{mg} / \mathrm{cm}^{2}$ & mylar + paper & 1) $285 \mathrm{mg} / \mathrm{cm}^{2}$ & Al \\
\hline DD & $25 \mathrm{mg} / \mathrm{cm}^{2}$ & NA $(i)$ & $x$ & $x$ & & 1) $25 \mathrm{mg} / \mathrm{cm}^{2}$ & paper & $\begin{array}{l}\text { 1) } 100 \mathrm{mg} / \mathrm{cm}^{2} \\
\text { 2) } 175 \mathrm{mg} / \mathrm{cm}^{2} \\
325 \mathrm{mg} / \mathrm{cm}^{2} \\
\text { 4) } 375 \mathrm{mg} / \mathrm{cm}^{2} \\
\text { 5) } 1660 \mathrm{mg} / \mathrm{cm}^{2}\end{array}$ & $\begin{array}{l}\text { plastic } \\
\text { plastic } \\
\text { plastic } \\
\text { plastic }\left(75 \mathrm{mg} / \mathrm{cm}^{2}\right) \\
\quad+\mathrm{Al} \\
\text { plastic }\left(140 \mathrm{mg} / \mathrm{cm}^{2}\right) \\
\quad+60 \% \mathrm{~Pb}+40 \% \mathrm{Sn}\end{array}$ \\
\hline $\mathrm{EE}$ & $\mathrm{U}$ & U & & & & NA & NA & NA & NA \\
\hline $\mathrm{FF}$ & $7 \mathrm{mg} / \mathrm{cm}^{2}$ & $1 \mathrm{~cm}$ & & $x$ & & $N A(h)$ & $N A(h)$ & $N A(h)$ & $N A(h)$ \\
\hline GG & u & u & & $x$ & & NA & paper & $\begin{array}{ll}\text { 1) } & 0.317 \mathrm{~cm} \\
\text { 2) } & 0.081 \mathrm{~cm} \\
\text { 3) } & 0.038 \mathrm{~cm} \\
\text { 4) } & 0.076 \mathrm{~cm}\end{array}$ & $\begin{array}{l}\text { plastic } \\
\text { Al } \\
C d+A l \\
P b+A l\end{array}$ \\
\hline $\mathrm{HH}$ & NA & NA & & & & 1) $20 \mathrm{mg} / \mathrm{cm}^{2}$ & plastic & $\begin{array}{l}\text { 1) } 0.9 \mathrm{~cm} \\
\text { 2) } 1.0 \mathrm{~cm}\end{array}$ & $\begin{array}{l}\mathrm{Pb} \\
\mathrm{Al}\end{array}$ \\
\hline I I & $7 \mathrm{mg} / \mathrm{cm}^{2}$ & U & $x$ & $x$ & $x$ & 1) $0 \mathrm{mg} / \mathrm{cm}^{2}$ & nothing & 1) $0.1 \mathrm{~cm}$ & cd \\
\hline Ju & $\mathrm{U}$ & u & & & & NA & NA & NA & NA \\
\hline KK & $7 \mathrm{mg} / \mathrm{cm} 2$ & U & & & & NA & NA & NA & NA \\
\hline LL & $10 \mathrm{mg} / \mathrm{cm}^{2}$ & $285 \mathrm{mg} / \mathrm{cm}^{2}$ & $x$ & & & 1) $10 \mathrm{mg} / \mathrm{cm}^{2}$ & mylar & NA & NA \\
\hline
\end{tabular}


TABLE 5. (Contd)

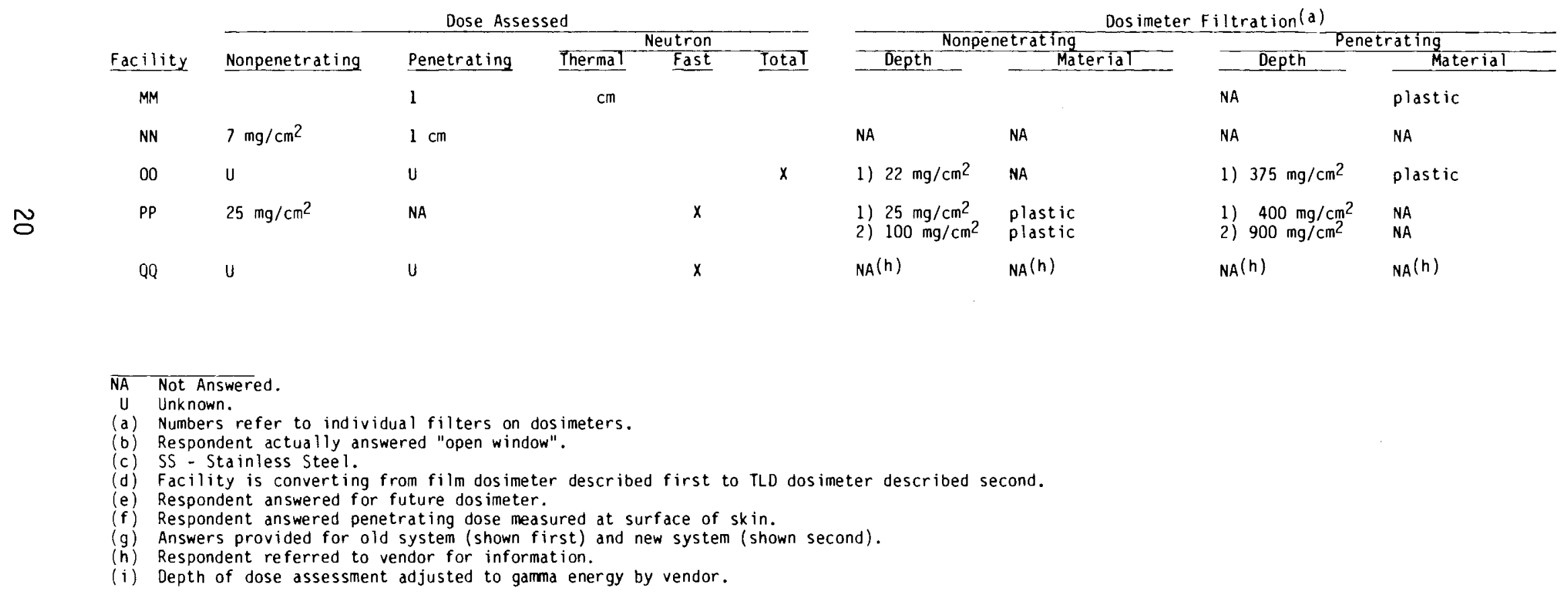




\section{QUALITY CONTROL}

PROCESS CONTROL DOSIMETERS

Several facilities reported using one or more types of process control dosimeters. The types asked in the questionnaire included as follows:

- Blank Check Dosimeters - simply routine dosimeters processed during the run which have received no dose.

- Pre-Dosed Dosimeters - typically dosimeters processed during the run which have been exposed to a specific dose (e.g., $1000 \mathrm{mrem}$ ).

- Open Audit - dosimeters known to be audit dosimeters but the dose is unknown.

- Blind Audit - dosimeters unknown to be audit dosimeters and, by implication, the dose is unknown.

Appendix $B$ allows inspection of quality control procedures for each facility. Many methods of process control are available to the dosimeter processor depending on reader design.

Figure 5 shows the percentage of facilities using the various types of process control dosimeters. Blank and pre-dosed check dosimeters are used extensively. Many of the facilities who employ check dosimeters or blind audit dosimeters also record the results. For some facilities, it was apparent that the use of process control dosimeters was conducted by the commercial vendor and not by the facility.

\section{$\underline{\text { RECORDS }}$}

The majority of facilities replied that DOE manual chapters were most responsible for the requirements of their personne 1 dosimetry records. As shown in Figure 5 , slightly more than $50 \%$ of the facilities replied that they could provide historical dosimetry policies. Slightly more than $50 \%$ of the facilities maintain historical records consistent with ANSI N13.6. (1) 


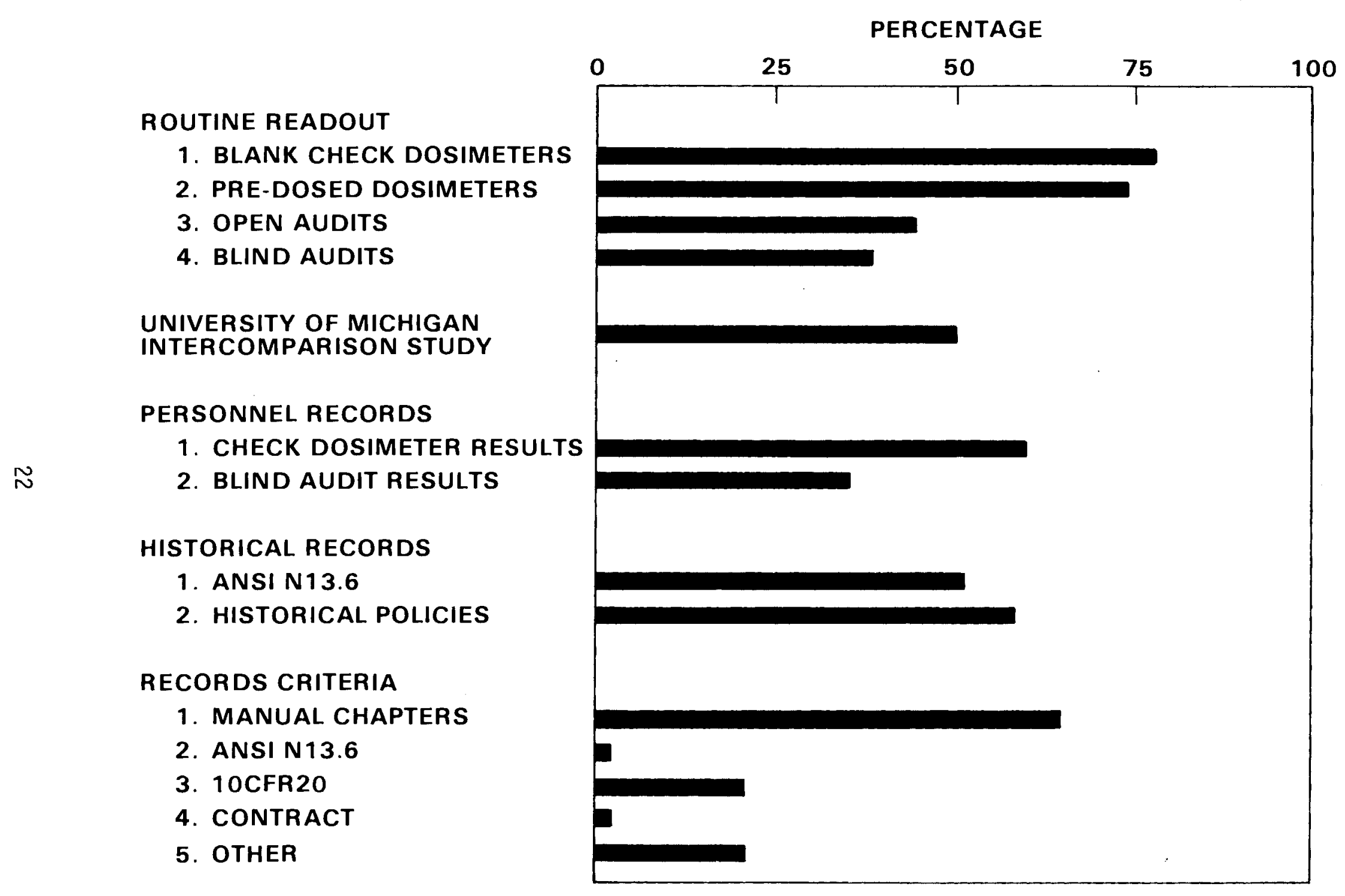

FIGURE 5. Percentage of Facilities Answering Yes 
INTERCOMPARISON STUDY

Twenty-one of the 43 facilities indicated that they or their commercial processor participated in the University of Michigan intercomparison study. (2) of these, only 3 facilities submitted the results of their performance. 


\section{ACKNOWLEDGMENT}

Special consideration is due the many people at the different DOE facilities who completed the questionnaire which form the basis of this report. Carmen Hudgens of PNL provided the graphical illustrations of each facilities input which comprise Appendix B. Marianna Cross typed the report and arranged for its publication. 


\section{REFERENCES}

1. American National Standards Institute. 1966. American National Standard Practice for Occupational Radiation Exposure Records Systems. N13.6, New York, New York.

2. Plato, P. and G. Hudson. 1980. "Performance Testing of Personnel Dosimetry Services," NUREG/CR-1064, Nuclear Regulatory Commission, Washington, D.C. 


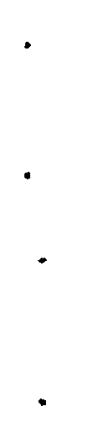




\section{APPENDIX A}

\section{PERSONNEL DOSIMETRY QUESTIONNAIRE AND RESPONSES}




\section{SECTION I}

1. Has the dosimeter in routine use been formally documented?

$$
\text { Yes }(a, b, c)
$$

No ${ }^{(d)}$

Not answered $(d, e)$

Document number or title provided

Yes

No

Reference to Proprietary Information 1

Reference to Vendor Documentation

2. Has the records system been documented?

Yes $(a, b, c)$

No ${ }^{(d)}$

Not answered $(c, d)$

Document number or title provided

Yes

No

Reference to Vendor Documentation

Reference to Proprietary Information

(a) One facility reported partially.

(b) One facility referenced state license.

(c) Two facilities referenced commercial service documentation.

(d) Five facilities referenced commercial service documentation.

(e) One facility receives beta-gamma dosimetry from a commercial vendor and neutron dosimetry from another DOE contractor facility. 
3. Has the dosimeter reader been documented?

$$
\text { Yes }(a, b)
$$

No

No answer

Document number or title provided

Yes

No

Vender procedures

9

Reference to proprietary information

4. Are routine procedures documented for

A) dosimeter calibration?

Yes

No

Not answered $(c)$

Vender information

B) dos imeter readout?

Yes

No

Not answered (c)

Vender information

c) dose calculation?

Yes (d)

27

No ${ }^{(e)}$

Not answered (c)

Vender information

2

\footnotetext{
(a) One facility referenced state license.

(b) Two facilities referenced commercial service documentation.

(c) Five facilities referenced commercial service documentation.

(d) One facility referenced computer code listings.

(e) One facility reported they do not have formally documented procedures but do have microfilmed records and laboratory notes on all calibration data, dosimeter readout, and dose calculations.
} 
D) personnel records?

$\begin{array}{lr}\text { Yes } & \\ \text { No } & 26 \\ \text { Not answered } & (b) \\ \text { Vender information } & 7 \\ & 2\end{array}$

5. Was the dosimeter designed by a DOE contractor?

Yes

No

Not answered

Contractor named

Yes

20

No

6. For the total dosimetry system, is the

A) dosimeter provided by a

1. DOE contractor?

Yes

21

No $(c, d)$

22

Contractor named?

Yes

2. Commercial service?

Yes $(e)$

22

No

20

Not answered 1

Vender named?

Yes

(a) One facility referenced descriptions of computer codes.

(b) One facility referenced commercial service documentation.

(c) One facility reported service provided by DOE laboratory.

(d) One facility is a DOE laboratory.

(e) Two facilities reported service from university radiation protection office. 
B) dosimeter readout provided by a

1. DOE contractor?

$\begin{array}{ll}\text { Yes } & 21 \\ \text { No } & (a, b) \\ 22\end{array}$

Contractor named?

Yes 16

2. Commercial service?

Yes (c) 21

No 21

Not answered 1

Vender named?

$\begin{array}{ll}\text { Yes } & 18\end{array}$

C) personnel records maintained by

1. DOE contractor?

Yes

No ${ }^{(b)}$

35

Not answered

Contractor named?

Yes

24

2. Commercial service?

Yes (c)

16

No 21

Not answered 6

Vendor named?

Yes 16

(a) One facility reported service provided by DOE laboratory.

(b) Three facilities are DOE laboratories.

(c) One facility reported service from university radiation protection office. 
7. How many man-years of record data do you have approximately? Information included in Table 3 of report.

8. About how many dosimeters were

A) processed in 1979?

Information included in Table 3 of report.

B) for how many people?

Information included in Table 3 of report. 


\section{SECTION II}

1. Is the present personnel dosimetry records system

A) on hard copy (paper)??

$$
\text { Yes }(a, b)
$$

$$
\text { No }(a)
$$

B) computerized?

$$
\begin{array}{lr}
\text { Yes }(b, c) & 26 \\
\text { No } & 15 \\
\text { Not answered } & 2
\end{array}
$$

C) on microfiche or microfilm?

$$
\begin{array}{lr}
\text { Yes }(d) & 12 \\
\text { No } & 28 \\
\text { Not answered } & 3
\end{array}
$$

2. Is information to the DOE respository at INEL provided by $(e)$

A) hard copy input?

$\begin{array}{lr}\text { Yes } & 26 \\ \text { No } & 14 \\ \text { Not answered } & 3\end{array}$

B) punched cards?

Yes 3

No 33

Not answered 7

C) SACNET?

Yes

No

Not answered

\footnotetext{
(a) One facility answered Yes and No.

(b) Two facilities answered partially.

(c) One facility reported computerized records at vender.

(d) One facility reported records on microfiche or microfilm by vender.

(e) One facility reported that they do not send records to repository.
} 
D) magnetic tape?

Yes

No

Not answered

3. Are annual dose summaries provided to employees

A) routinely?

Yes $15^{(a, b)}$

No

Not answered

B) on request?

Yes

No

4. Is the cumulative radiation dose history requested

A) for new employees?

$\begin{array}{lr}\text { Yes (c) } & 35 \\ \text { No } & (d) \\ & 8\end{array}$

B) if so, is the dose history required prior to allowing new employees to work in a radiation zone?

Yes $(e, f)$

No $(g)$

(a) One facility started in 1980 (1979 year reported).

(b) One facility provides quarterly dose summaries.

(c) One facility requested previous exposure if employee indicates receipt of previous exposure and job assignement included radiation zone.

(d) One facility reported that previous dose history is obtained if employee indicates high exposure was received.

(e) One facility reported yes if employee advises exposure received in excess of permissible limits during previous employment (answered no in 4.A).

(f) One facility reported a case by case review but did not state the criteria.

(g) One facility reported 7 of 8 contractors at site did not require dose history prior to a new employee entering a radiation zone; one did require the information. 
5. Are employee job assignments in radiation zones documented such that duration of assignment, job task, and exposure information are available?

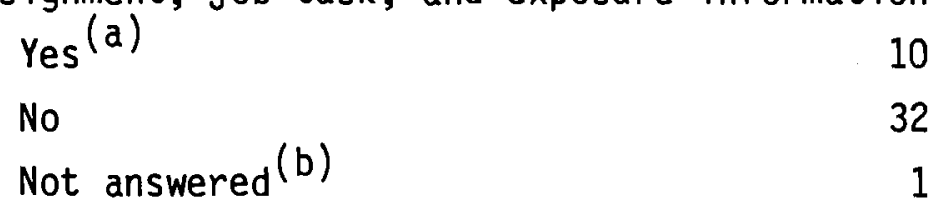

6. Is the DOE repository used to determine new employee past cumulative radiation exposure at DOE sites?

$\begin{array}{lr}\text { Yes } & 1 \\ \text { No }(c) & 42\end{array}$

(a) One facility reported information in area access logs kept for radiation area control.

(b) One facility referenced some records maintained by individual facilities. None kept in dosimetry records.

(c) One facility reported they were never issued instructions on the procedure to query the repository. 
7. What parameters are included in the individual dosimetry records?

A) Name

Yes 43

No

Not answered

B) Birthdate

Yes (a)

39

No

4

Not answered

C) Sex

Yes $^{(b)}$

No

Not answered

D) Social security number

Yes

No

Not answered

E) Payroll number

Yes (c)

No

22

Not answered

(a) One facility reported birthdate available since 1972.

(b) One facility reported information available from personnel records.

(c) Three facilities reported unique identification numbers different from payroll numbers. 
F) Occupation

Yes $^{(a)} \quad 12$

No 31

Not answered

G) Hire date

Yes 26

No ${ }^{(b)} \quad 17$

Not answered

H) Facility type (DOE Form 190)

Yes

22

No 21

Not answered

I) Previous exposure total

Yes $(c, d) \quad 30$

No 13

Not answered

J) Internal depositions:

1) systemic burden

Yes (e)

No

Not answered

Not applicable

(a) One facility reported by department (e.g., maintenance, mechanic, or laboratory technician) and facility as well as supervisory or worker.

(b) One facility reported information available from personnel records.

(c) One facility reported yes for previous employment since 1977.

(d) One facility reported yes if a previous high exposure was received.

(e) One facility reported yes for Pu in urine assay data. 
2. percent of MPBB

$\begin{array}{lr}\text { Yes } & 13 \\ \text { No } & 25 \\ \text { Not answered } & 3 \\ \text { Not applicable } & 2\end{array}$

3. percent of organ burden

Yes $(a, b, c) \quad 14$

No 23

Not answered 4

Not applicable 2

K) Intake date for deposition

$\mathrm{Yes}(\mathrm{d}, \mathrm{e})$

No 18

Not answered 1

Not applicable, 2

L) Organ of deposition

Yes $(a, c) \quad 25$

No 15

Not answered 1

Not applicable 2

M) Radionuclide of deposition

Yes 30

No ${ }^{(e)} \quad 10$

Not answered 1

Not applicable 2

(a) One facility answered yes for 1 only.

(b) One facility reported yes for in vivo chest counts only.

(c) One facility reported yes for thyroid only.

(d) One facility reported yes for Pu and $\mathrm{H}-3$ uptakes.

(e) One facility reported data in laboratory data not records systems. 
N) Annual dose from deposition

$$
\text { Yes }(a, b, c)
$$

No

Not answered

Not applicable

0) Method of analys is

$\begin{array}{lr}\text { Yes } & 26 \\ \text { No }(d) & 14 \\ \text { Not answered } & 1 \\ \text { Not applicable } & 2\end{array}$

P) Dosimeter data:

1. nonpenetrating

Yes

No $(c)$

2. penetrating

$\begin{array}{lr}\text { Yes } & 40 \\ \text { No } & \end{array}$

3. neutron total (separate from beta, gamma data)

Yes

No $(e)$

Not applicable

4. slow neutrons

Yes

No

(a) One facility answered yes for lung only.

(b) One facility reported yes for $\mathrm{H}-3$ only.

(c) One facility reported quarterly dose determination.

(d) One facility reported records kept in laboratory results file.

(e) One facility reported yes and indicated raw data not kept on file. Response was recorded as a no. 
5. fast neutrons

$\begin{array}{lr}\text { Yes } & 23 \\ \text { No } & 15 \\ \text { Not answered } & 1 \\ \text { Not applicable } & 4\end{array}$

6. high LET radiations

Yes 1

No 35

Not answered 1

Not applicable 6

type specified

protons 1

Q) Dosimeter dose:

1. whole-body

Yes 43

2. skin

Yes $\quad 36$

No 7

3. extremity

Yes $\quad 34$

No ${ }^{(a)} \quad 9$

(a) One facility records dose for those who wear finger rings, only. 
R) Dosimeter exchange frequency

$\begin{array}{lc}\text { Yes } & (a) \\ \text { No } & 35 \\ \text { Not answered } & 6 \\ \text { Other } & 2 \\ \end{array}$

8. Is historical information maintained regarding worker

A) craft?

$$
Y_{e s}(b, c, d)
$$

No

B) job assignment (facility)?

$$
\text { Yes (c) }
$$

No ${ }^{(b)}$

9. Does the present records system provide information directly regarding

A) employees currently terminating (DOE Form 192)?

$\begin{array}{ll}\text { Yes } & 32 \\ \text { No } & 11\end{array}$

B) statistical annual whole-body dose distribution for current employees (DOE Form 190) by 1. facility type?

(a) One facility records month of dosimeter issue and return.

(b) One facility maintains craft and facility assignments in general. For example, a maintenance mechanic assigned to a reactor area can be identified but they cannot easily determine if the mechanic worked elsewhere.

(c) One facility answered yes but indicated only current status maintained.

(d) Two facilities answered generally yes -- information is contained in contractor personnel files.

(e) One facility answered yes because entire facility is a single classification. 
2. occupational class?

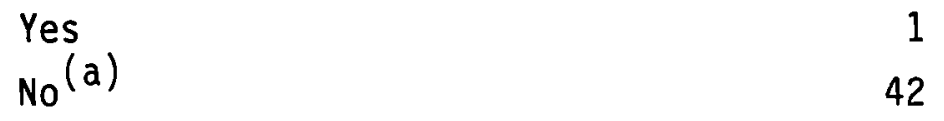

C) statistical cumulative lifetime whole-body dose distribution for current employees by

1. facility type?

$$
\begin{aligned}
& \text { Yes } \\
& \mathrm{No}^{(\mathrm{c})}
\end{aligned}
$$

2. occupational class?

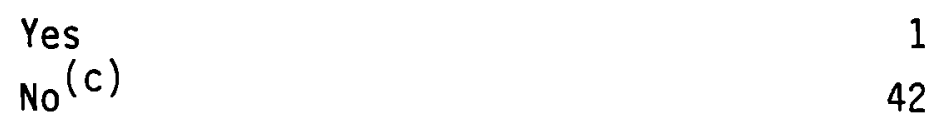

D) age versus dose distribution for current employees for 1. annual dose?

$\begin{array}{lr}\text { Yes }^{(a)} & 2 \\ \text { No } & 41\end{array}$

2. lifetime cumulative dose?

$\begin{array}{lr}\text { Yes } & (b) \\ \text { No } & 2 \\ & 41\end{array}$

\footnotetext{
(a) One facility reported yes for current year only.

(b) One facility reported yes for doses received at that facility.

(c) One facility reported that information could be obtained if needed.
} 
E) sex versus dose distribution for current employees for

1. annual dose?

$$
\begin{aligned}
& Y_{\text {Yes }}(a, b) \\
& \text { No }^{(c, d)}
\end{aligned}
$$

2. lifetime cumulative dose?

$$
\begin{array}{lr}
\text { Yes } & 1 \\
\mathrm{No}_{\mathrm{O}}(c, d) & 42
\end{array}
$$

F) sex and age versus dose distribution for current employees for 1. annual dose?

$$
\begin{array}{lr}
\text { Yes }^{(b)} & 1 \\
\mathrm{No}^{(c)} & 42
\end{array}
$$

2. lifetime cumulative dose?

$$
\begin{array}{lr}
\text { Yes } & 1 \\
\text { No } & (c) \\
& 42
\end{array}
$$

G) past terminated employees, such as

1. list of names?

$\begin{array}{ll}\text { Yes } & 27 \\ \text { No } & 16\end{array}$

2. annual dose by year?

Yes 30

No 13

(a) One facility reported yes for child bearing aged females.

(b) One facility reported yes for current year only.

(c) One facility reports information available upon special request but not done routinely.

(d) One facility reported information could be obtained if needed. 
3. depositions by year?

$\begin{array}{ll}\text { Yes } & \\ \text { No } & 13 \\ & 30\end{array}$

H) craft versus dose distribution for current employees

1. annual dose?

$$
\text { Yes }(b, c)
$$

No $(d)$

2. lifetime cumulative dose?

$$
\text { Yes (b) }
$$

No (d)

I) quality control dosimeters

1. readout check results?

Yes

No

2. blind audit results?

Yes

No

Not answered (e)

(a) One facility reported yes for Pu only.

(b) One facility maintains records for various groups of employees which includes individual doses since employment at facility.

(c) One facility reported yes for current year only.

(d) One facility reports information available upon special request but not done routinely.

(e) One facility referenced vender quality control dosimeter usage. 
10. Are historical occupational radiation records as described in ANSI N13.6 maintained (i.e., historical dosimetry records and policies)?

Yes

No

Not answered
22

15

6

11. Can policies be reconstructed for any historical date?

Yes ${ }^{(a)}$

No

Not answered
12. What criteria are used to retain records?

A) manual chapters

B) ANSI 13.6

C) Code of F.R. for Licensees

D) contract

E) Other

Indefinite retention of individual cumulative dose

Internal procedures

Records retained indefinitely

Privacy Act

Corporate Policy

F) Not answered

\section{1}

9

1

1

4

2

1

1

2

(a) One facility answered yes with some exceptions. 
13. Is there a lower dose threshold below which all doses are considered background for

A) nonpenetrating?

Yes

No

Not answered

Not applicable

dose specified

$\begin{array}{ll}5 \text { mrem } & 5 \\ 9 \text { mrem } & 1\end{array}$

$10 \mathrm{mrem} \quad 12$

15 mrem 2

20 mrem 4

$30 \mathrm{mrem} \quad 1$

40 mrem 2

$1.6 \mathrm{mrem} / \mathrm{wk}+20$

$40 \mathrm{mrad} / \mathrm{fj} 1 \mathrm{~m}$

B) penetrating?

Yes

No

Not answered 2

Not applicable 1

dose specified

$\begin{array}{lr}5 \text { mrem } & 5 \\ 10 \mathrm{mrem} & 17 \\ 15 \mathrm{mrem} & 2 \\ 30 \mathrm{mrem} & 4 \\ 40 \mathrm{mrem} & 2 \\ 1.6 \mathrm{mrem} / \mathrm{wk}+20 & 1 \\ 30 \mathrm{mR} / \mathrm{film} & 1\end{array}$


c) neutron?

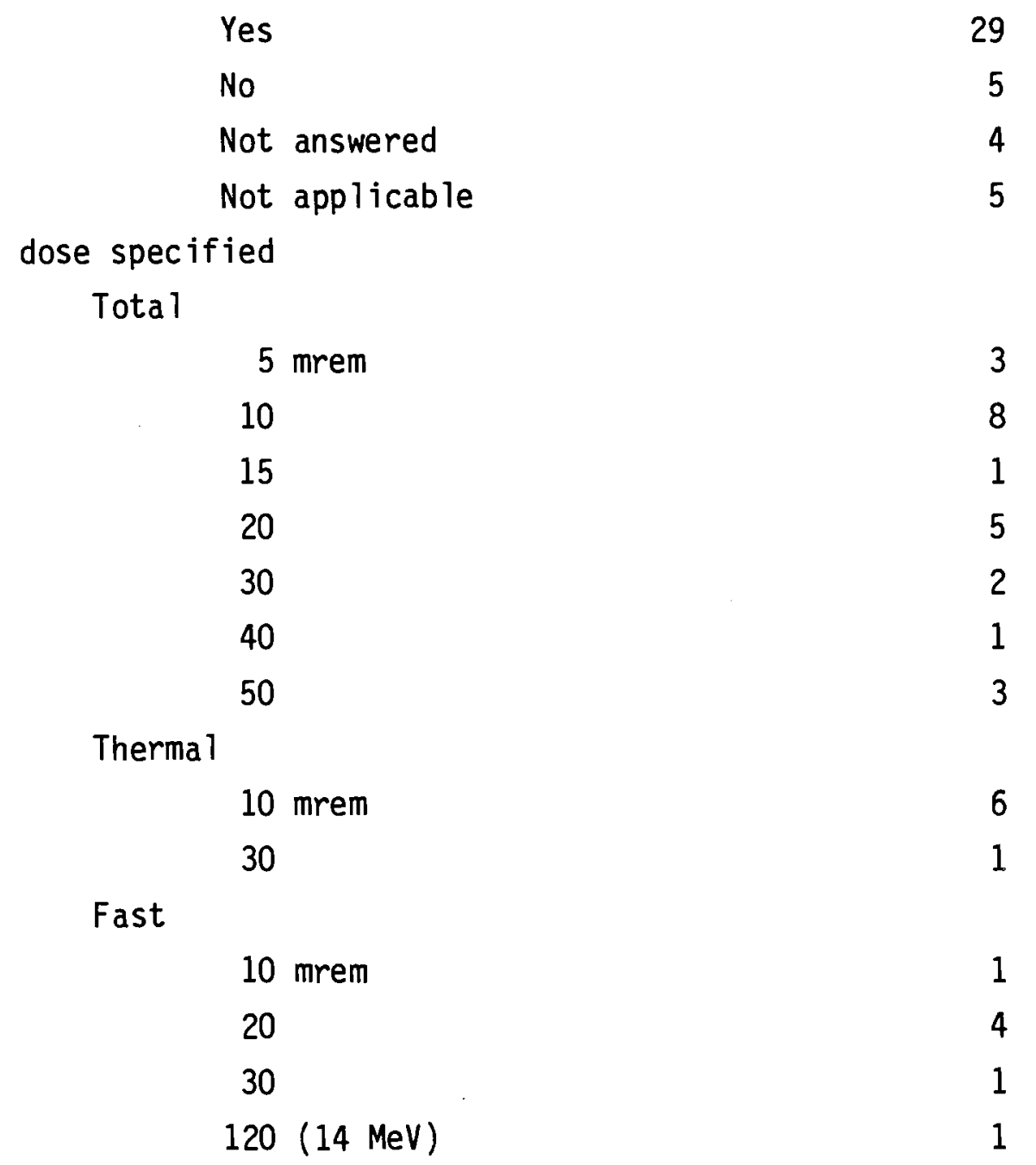

14. Is dose contribution from background radiation subtracted from occupationa 1 dose?

$\begin{array}{lr}\text { Yes } & 35 \\ \text { No } & 6 \\ \text { Not answered } & 2\end{array}$


15. Is skin dose calculated by (a)

A) adding penetrating and nonpenetrating doses together?

$\begin{array}{lr}\text { Yes } & 20 \\ \text { No } & 20 \\ \text { Not answered } & 1 \\ \text { Not applicable } & 2\end{array}$

B) dose conversion from nonpenetrating component only? Yes 9

No 28

Not answered 4

Not applicable 2

C) dose conversion from penetrating and nonpenetrating components?

Yes 5

No 30

Not answered 6

Not applicable 2

16. Dosimeter type in routine use

A) beta-gamma

1. TLD

$\begin{array}{lr}\text { Yes }(b, c, d) & 29 \\ \text { No } & 13 \\ \text { Not answered } & 1\end{array}$

2. $f i l m$

Yes 27

No 13

Not answered 3

(a) One facility reported the ir method which consisted of adding nonpenetrating which includes penetrating, neutron, and tritium doses to estimate skin dose.

(b) One facility reported TLD used only as an accident dosimeter.

(c) Two facilities reported use of TLD for extremities only.

(d) One facility used TLD only for special applications. 
3. ionization

$\begin{array}{lr}\text { Yes }(a, b) & 9 \\ \text { No } & 28 \\ \text { Not answered } & 6\end{array}$

B) neutron

1. TLD

$\begin{array}{ll}\text { Yes } & 18\end{array}$

$\begin{array}{ll}\text { No } & 18\end{array}$

Not answered 3

Not applicable 4

2. film

$\begin{array}{ll}\text { Yes } & 18\end{array}$

$\begin{array}{ll}\text { No } & 17\end{array}$

Not answered 4

Not applicable 4

3. ionization

Yes

No 32

Not answered $\quad 7$

Not applicable 4

4. other

a. One facility reported using track etch

b. Two facilities reported track etch under development

(a) One facility reported use of pocket ionization chambers for radiation area entry exposure control.

(b) One facility reported use of ionization dosimeters for special applications only. 
17. Does the personnel dosimeter(s) in routine use measure A) nonpenetrating dose at $7 \mathrm{mg} / \mathrm{cm}^{2}$ ?

Yes

No

Unknown (a)

Not answered

Not applicable

other depths specified

$10 \mathrm{mg} / \mathrm{cm}^{2}$

$15 \mathrm{mg} / \mathrm{cm}^{2}$

$23 \mathrm{mg} / \mathrm{cm}^{2}$

$25 \mathrm{mg} / \mathrm{cm}^{2}$

$32 \mathrm{mg} / \mathrm{cm}^{2}$

$52 \mathrm{mg} / \mathrm{cm}^{2}$

Surface dose relative to depleted uranium metal

Film calibrations from specific isotope of concern

(a) One facility referred to vender information. 
B) penetrating dose at $1 \mathrm{~cm}$ ?

Yes

No (a)

13

Unknown (b)

14

Not answered

12

4

penetrating dose at $5 \mathrm{~cm}$ ?

Yes

2

No

18

Unknown

11

Not answered

12

other depths specified

evaluated from $C d$ ratio 1

$270 \mathrm{mg} / \mathrm{cm}^{2}$

1

$1400 \mathrm{mg} / \mathrm{cm}^{2}$

1010 and $1400 \mathrm{mg} / \mathrm{cm}^{2}$

1

$2 \mathrm{~cm}$

gamma relative to ${ }^{60} \mathrm{Co}$

measured at surface of skin

$322 \mathrm{mg} / \mathrm{cm}^{2}$

dose dependent on energy

$285 \mathrm{mg} / \mathrm{cm}^{2}$

adjusted to gamma energy (vendor)

(a) One facility plans to measure penetrating dose at $1 \mathrm{~cm}$ in the future. (b) One facility referred to vender information. 
C) neutron dose, slow?

$\begin{array}{cr}\text { Yes } & 12 \\ \text { No } & 15 \\ \text { Not answered } & 12 \\ \text { Not applicable } & 4 \\ \text { neutron dose, fast? } & 22 \\ \text { Yes } & 10 \\ \text { No } & 8 \\ \text { Not answered } & 3 \\ \text { Not applicable } & \\ \text { neutron dose, total? } & 19 \\ \text { Yes } & 12 \\ \text { No } & 9 \\ \text { Not answered } & 3 \\ \text { Not applicable } & \end{array}$

18. What quality factors are used to determine

A) slow neutron dose?

3

$1-3$

6

2

3.5-5

1

Unknown 2

101

Flux to dose factors from NCRP \#38 4

Films calibrated for \# of $\mathrm{tracks} / \mathrm{cm}^{2} / \mathrm{rem}$

10 CFR, Table 10 
B) fast neutron dose?

10

$5.7 \quad 1$

Flux to dose factors from NCRP \#38 4

Individually measured calibrated

factors

1

C) total neutron dose?

10

2

2-11

1

6-10 1

Flux to dose factors from NCRP \#38 3

Individually measured calibrated factors 3

Manual chapter 0524, Part 4

Albedo response curve and $3^{\prime \prime} / 9^{\prime \prime}$ sphere ratios 1

$1 \mathrm{mRem}=2.6 \times 10^{4} \mathrm{~N} / \mathrm{cm}^{2} \quad 1$ 
19. What is the filtration on dosimeter for A) nonpenetrating

$$
\begin{aligned}
\text { Facility A } & \text { Filter \#1, } 25 \mathrm{mg} / \mathrm{cm}^{2} \text {, paper } \\
\text { B } & \text { Not answered } \\
\text { C } & \text { Variable } \\
\text { D } & \text { Filter } \# 1,40 \mathrm{mg} / \mathrm{cm}^{2} \text {, plastic } \\
\text { E } & \text { Filter } \# 1,50 \mathrm{mg} / \mathrm{cm}^{2} \text {, plastic } \\
& \# 2,150 \mathrm{mg} / \mathrm{cm}^{2} \text {, plastic } \\
\text { F } & \text { Filter } \# 1,7 \mathrm{mg} / \mathrm{cm}^{2}, \text { plastic } \\
\text { G } & \text { Filter } \# 1,5.6 \mathrm{mg} / \mathrm{cm}^{2} \text {, plastic } \\
\text { H } & \text { Dosimeters used: }
\end{aligned}
$$

\section{Facility $D$ (thermal neutron)}

2. Landauer (beta-gamma, fast neutron)

Landauer $\mathrm{G}-1, \mathrm{E}-1$, and $\mathrm{U}-3$ dosimeters

I Filter $\# 1,32 \mathrm{mg} / \mathrm{cm}^{2}$, cellulose acetate

J Filter $\# 1,7 \mathrm{mg} / \mathrm{ccm}^{2}$, plastic

$\mathrm{K}$ Filter \#1, $23 \mathrm{mg} / \mathrm{cm}^{2}$, plastic

L Filter \#1, $80 \mathrm{mg} / \mathrm{cm}^{2}$, plastic + paper

M Filter \#1, $15 \mathrm{mg} / \mathrm{cm}^{2}$, teflon

$\mathrm{N}$ Filter \#1, $12 \mathrm{mg} / \mathrm{cm}^{2}, \mathrm{Al}$

0 Filter \#1, $60 \mathrm{mg} / \mathrm{cm}^{2}$, plastic

P Filter \#1, $4.5 \mathrm{mg} / \mathrm{cm}^{2}$, mylar

Q Filter \#1, $80 \mathrm{mg} / \mathrm{cm}^{2}$, plastic

R Filter $\# 1,20-30 \mathrm{mg} / \mathrm{cm}^{2}$, plastic

$\# 2,130 \mathrm{mg} / \mathrm{cm}^{2}, \mathrm{Al}$

$\# 3,130 \mathrm{mg} / \mathrm{cm}^{2}, \mathrm{Ag}$

S Filter \#1, $103 \mathrm{mg} / \mathrm{cm}^{2}$, plastic + paper

$\# 2,1087 \mathrm{mg} / \mathrm{cm}^{2}$, plastic + tantalum

+ cellulose acetate + paper

\#3, $1065 \mathrm{mg} / \mathrm{cm}^{2}$, plastic + Cd $+\mathrm{Ta}$

+ cellulose acetate + paper

\#4, $507 \mathrm{mg} / \mathrm{cm}^{2}$, plastic

+ cellulose acetate + paper 
Facility T Filter $\# 1,52 \mathrm{mg} / \mathrm{cm}^{2}$, plastic

$U$ Not answered

$\checkmark$ Not answered

W Filter \#1, $55.3 \mathrm{mg} / \mathrm{cm}^{2}$, plastic

\#2. $23.7 \mathrm{mg} / \mathrm{cm}^{2}$, paper

$X \quad$ Filter $\# 1,7 \mathrm{mg} / \mathrm{cm}^{2}$, plastic

$Y$ Filter \#1, Open Window

\#2, $100 \mathrm{mg} / \mathrm{cm}^{2}$, plastic

$\# 3,175 \mathrm{mg} / \mathrm{cm}^{2}$, plastic

$\# 4,325 \mathrm{mg} / \mathrm{cm}^{2}$, plastic

$\# 5,375 \mathrm{mg} / \mathrm{cm}^{2}, \mathrm{~A} 1$

$\# 6,660 \mathrm{mg} / \mathrm{cm}^{2}, \mathrm{~Pb}+\mathrm{Sn}$

$Z$ Not answered

AA Filter \#1, $25 \mathrm{mg} / \mathrm{cm}^{2}$, plastic

$\# 2,100 \mathrm{mg} / \mathrm{cm}^{2}$, plastic

$\# 3,175 \mathrm{mg} / \mathrm{cm}^{2}$, plastic

\#4, $325 \mathrm{mg} / \mathrm{cm}^{2}$, plastic

BB Not answered - referred to vender

CC Filter \#1, $10 \mathrm{mg} / \mathrm{cm}^{2}$, mylar + paper

DD Filter \#1, $25 \mathrm{mg} / \mathrm{cm}^{2}$, paper

$\# 2,100 \mathrm{mg} / \mathrm{cm}^{2}$, plastic

\#3, $175 \mathrm{mg} / \mathrm{cm}^{2}$, plastic

$\# 4,325 \mathrm{mg} / \mathrm{cm}^{2}$, plastic

$\# 5,375 \mathrm{mg} / \mathrm{cm}^{2}$, plastic $+\mathrm{A} 1$

$\# 6,1660 \mathrm{mg} / \mathrm{cm}^{2}$, plastic $+\mathrm{Pb}+\mathrm{Sn}$

EE Not answered

FF Referred to vender

GG Paper wrapper

$\mathrm{HH}$ Filter $\# 1,20 \mathrm{mg} / \mathrm{cm}^{2}$, plastic

II No filter

JJ Not answered

KK Not answered

LL Filter \#1, $10 \mathrm{mg} / \mathrm{cm}^{2}$, mylar

MM Not answered 
Facility NN Not answered

$$
\begin{array}{r}
00 \text { Filter \#1, } 22 \mathrm{mg} / \mathrm{cm}^{2} \text {, plastic } \\
\text { PP Filter \#1, } 25 \mathrm{mg} / \mathrm{cm}^{2} \text {, plastic } \\
\# 2,100 \mathrm{mg} / \mathrm{cm}^{2} \text {, plastic }
\end{array}
$$

QQ Not answered

B) penetrating?

$$
\begin{aligned}
& \text { Facility A } \text { Filter } \# 1,0.3 \mathrm{~cm} \text {, plastic } \\
& \# 2,0.1 \mathrm{~cm}, \mathrm{Al}+0.5 \mathrm{~cm} \text {, plastic } \\
& \# 3,0.15 \mathrm{~cm}, \mathrm{Sn}+\mathrm{Pb}+0.14 \mathrm{~cm} \text {, plastic } \\
& \text { B } \quad \text { Filter } \# 1,0.028 \mathrm{~cm}, \mathrm{Cd} \\
& \text { C } \quad \text { Variable } \\
& \text { D Filter } \# 1,93 \mathrm{mg} / \mathrm{cm}^{2}, \text { brass } \\
& \text { E Filter } \# 1,1000 \mathrm{mg} / \mathrm{cm}^{2}, A 1+\text { plastic } \\
& \text { F } \quad \text { Filter } \# 1,0.1 \mathrm{~cm}, \mathrm{Al} \\
& \text { G Filter } \# 1,0.17 \mathrm{~cm}, A 1 \\
& \text { H } \quad \text { Dosimeters used: }
\end{aligned}
$$

1. Facility $D$ (thermal neutron)

2. Landauer (beta-gamma, fast neutron) Landauer $G-1, E-1$, and $U-3$ dosimeters

I Filter \#1, $1400 \mathrm{mg} / \mathrm{cm}^{2}$, plastic

J Filter \#1, $0.0025 \mathrm{~cm}$, brass

K Filter \#1, $1400 \mathrm{mg} / \mathrm{cm}^{2}, \mathrm{Cd}+\mathrm{SS}$

$\# 2,462 \mathrm{mg} / \mathrm{cm}^{2}, \mathrm{Al}$

L Filter \#1, $0.076 \mathrm{~cm}, \mathrm{Cd}$

$\# 2,0.013 \mathrm{~cm}$, Au

$\# 3,0.081 \mathrm{~cm}$, plastic + paper

M Filter \#1, $0.127 \mathrm{~cm}, \mathrm{Al}$

$N$ Not answered

0 Filter \#1, $90 \mathrm{mg} / \mathrm{cm}^{2}, \mathrm{Cu}$

$$
+250 \mathrm{mg} / \mathrm{cm}^{2} \text {, plastic }
$$

$P \quad$ Filter \#1, $0.2 \mathrm{~cm}, \mathrm{~A} 1$

Q Filter $\# 1,80 \mathrm{mg} / \mathrm{cm}^{2}$, plastic

$\# 2,93 \mathrm{mg} / \mathrm{cm}^{2}$, plastic

$$
+173 \mathrm{~cm}^{2}, \mathrm{Al}
$$


Facility R Filter $\# 1,0.1 \mathrm{~cm}, \mathrm{Cd}$

\#2, $0.02 \mathrm{~cm}$, plastic

$S$ Filter \#1, $103 \mathrm{mg} / \mathrm{cm}^{2}$, plastic + paper

$\# 2,1087 \mathrm{mg} / \mathrm{cm}^{2}$, plastic + tantalum

+ cellulose acetate + paper

\#3, $1065 \mathrm{mg} / \mathrm{cm}^{2}$, plastic $+\mathrm{Cd}+\mathrm{Ta}$

+ cellulose acetate + paper

\#4, $507 \mathrm{mg} / \mathrm{cm}^{2}$, plastic

+ cellulose acetate + paper

T Filter \#1, $52 \mathrm{mg} / \mathrm{cm}^{2}$, plastic

$\# 2,270 \mathrm{mg} / \mathrm{cm}^{2}, \mathrm{Al}$

$\checkmark \quad$ Not answered

$\checkmark$ Not answered

W Filter \#1, $0.051 \mathrm{~cm}$, plastic

$+0.038 \mathrm{~cm}, \mathrm{Cd}$

$+0.013 \mathrm{~cm}, \mathrm{Au}$

$+0.038 \mathrm{~cm}, \mathrm{Cd}$

$+0.066 \mathrm{~cm}$, plastic

$+0.025 \mathrm{~cm}$, paper

$X$ Filter \#1, $1 \mathrm{~cm}, \mathrm{Al}$

$Y$ Filter \#1, Open Window

\#2, $100 \mathrm{mg} / \mathrm{cm}^{2}$, plastic

$\# 3,175 \mathrm{mg} / \mathrm{cm}^{2}$, plastic

\#4, $325 \mathrm{mg} / \mathrm{cm}^{2}$, plastic

$\# 5,375 \mathrm{mg} / \mathrm{cm}^{2}, \mathrm{~A} 1$

$\# 6,660 \mathrm{mg} / \mathrm{cm}^{2}, \mathrm{~Pb}+\mathrm{Sn}$

Z Not answered

AA Filter \#1, $25 \mathrm{mg} / \mathrm{cm}^{2}$, plastic

\#2, $100 \mathrm{mg} / \mathrm{cm}^{2}$, plastic

$\# 3,175 \mathrm{mg} / \mathrm{cm}^{2}$, plastic

\#4, $325 \mathrm{mg} / \mathrm{cm}^{2}$, plastic

$B B$ Not answered - referred to vender

CC Filter \#1, $285 \mathrm{mg} / \mathrm{cm}^{2}, \mathrm{~A}$ ] 


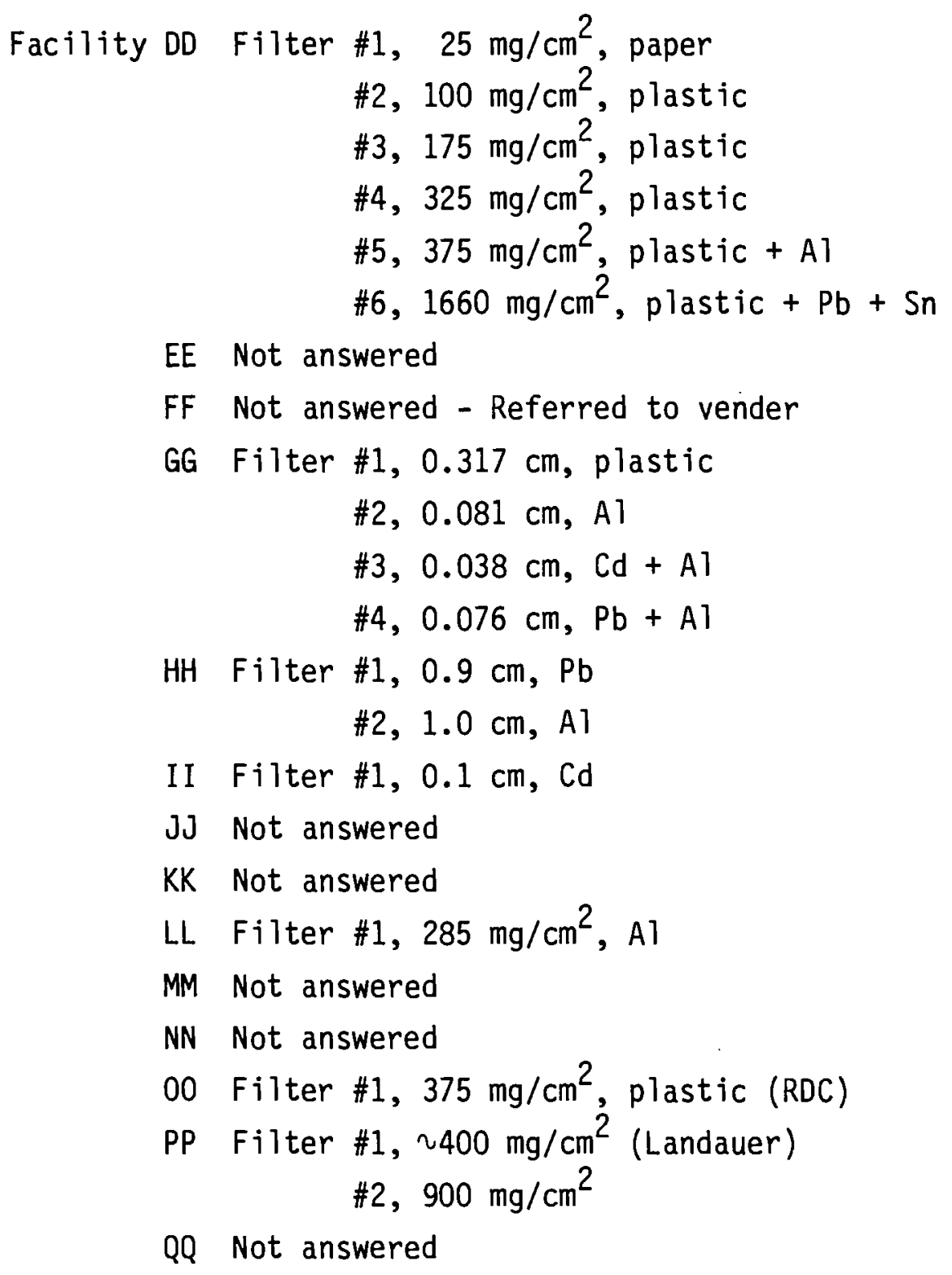


20. During routine dosimeter readout are

A) blank check dosimeters read?

$$
\text { Yes (a) }
$$

No

Not answered (b)

B) pre-dosed (e.g., 1.OR) check dosimeters read? Yes (a)

No

Not answered (b)

4

7

C) open audit dosimeters read?

Yes (a) 19

No

16

Not answered (b)

D) blind audit dosimeters read?

Yes (a) 16

No 21

Not answered 6

21. Was dosimeter system included in intercomparison study at the University of Michigan?
Yes $(c)$
21
No
18
Not answered (d)
4

results provided?

Yes

(a) Two facilities referred to commercial processor procedures which included yes answered to $20 \mathrm{~A}, \mathrm{~B}, \mathrm{C}$, and $\mathrm{D}$.

(b) Three facilities referred to vendor.

(c) Two facilities reported participation by commercial vender.

(d) Two facilities referred to vender. 
22. Is combined dose to organ(s) from external radiation and internal deposition(s)

A) routinely assessed?

Yes

No

26

Not answered

Not applicable

B) assessed when internal deposition measurable?

Yes

21

No

16

Not answered

3

Not applicable

3

C) assessed when internal dose exceeds $\%$ (please specify) of annual dose limit?

Yes $(a, b)$

9

No

18

Not answered

13

Not applicable

3

specified percents

$1 \%$

2

$5 \%$

1

$50 \%$

1

$67 \%$

1

$25 \%$ of quarterly limit

Whenever 50 year dose commitment

1

exceeds 0.1 of 0524 quarter limit

(a) One facility reported combined dose assessed when dose to lungs from external radiation and internal deposition equals or exceeds $40 \%$ of dose limit.

(b) One facility reported yes and percentage varies according to nuclide. 
D) assessed when internal deposition exceeds $\%$ (please specify) of maximum permissible organ burden?

$\begin{array}{ll}\text { Yes }(a, b) & 8 \\ \text { No } & 16 \\ \text { Not answered } & 16 \\ \text { Not applicable } & 3\end{array}$

specified percent

$\begin{array}{rr}1 \% & 1 \\ 5 \% & 3 \\ 50 \% & 1 \\ 67 \% & 1\end{array}$

E) assessed on an individual basis?

$\begin{array}{lr}\text { Yes }(c) & 31 \\ \text { No }(c) & 6 \\ \text { Not answered } & 3 \\ \text { Not applicable } & 3\end{array}$

F) not assessed because internal doses are not significant?

Yes

No

21

Not answered

11

Not applicable

F) not assessed?

Yes

No (c)

23

Not answered (c)

14

Not applicable

(a) One facility reported combined dose assessed when dose to lungs from external radiation and internal deposition equals or exceeds $40 \%$ of dose limit.

(b) One facility reported yes and percentage varies according to nuclide.

(c) One facility reported yes and no answers 
23. For internal depositions detected during routine in vivo or bioassay analyses, what date is assumed for the deposition?

A) day following last analysis?

Yes

No

19

Not answered

Not applicable

B) mid-point of time period since last analys is?

$$
\text { Yes }(a, b)
$$

No

Not answered

Not applicable

C) current date?

$\begin{array}{lr}\text { Yes }^{(b)} & 3 \\ \text { No } & 22 \\ \text { Not answered } & 15 \\ \text { Not applicable } & 3\end{array}$

(a) Assumed only if date cannot be determined by investigation for one facility.

(b) One facility reported yes to 23B) for $\mathrm{Pu}$ only and yes to 23C) for $\mathrm{H}-3$ only. 
D) Other answers

1. When person was last involved in an activity which could have included the deposition.

2. Each case studied individually - most likely date assigned.

3. Each case studied individually - midpoint of time period since last analys is assumed only if date cannot be determined.

4. Investigation determines deposition date. If not possible, then $23 \mathrm{~A}$ assumed.

5. Exposures are chronic and, therefore, average burdens are calculated on an annual basis.

6. Invivo examination scheduled only when internal deposition is suspected. Therefore, day of deposition is known. 
APPENDIX B

GRAPHICAL REPRESENTATION OF RESPONSES BY FACILITY 
FACILITY - A

TYPE - NATIONAL LABORATORY

DOSIMETRY VOLUME - LARGE

DOSIMETRY SERVICE - COMMERCIAL

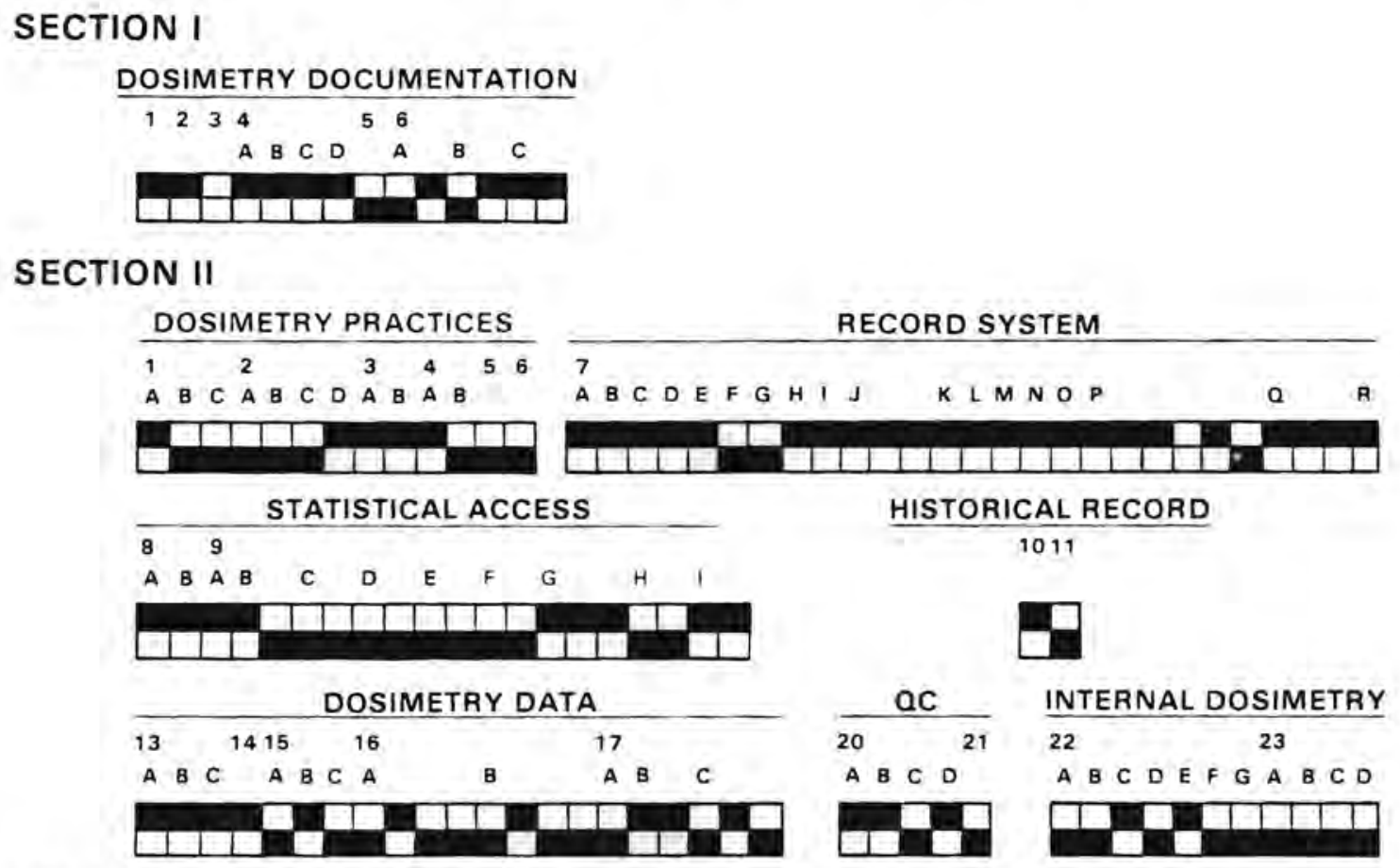

\section{SPECIFIC QUESTIONS AND ANSWERS}

\#12. DOE MANUAL CHAPTERS

\#17. A. NONPENETRATION DOSE AT $=25 \mathrm{mg} \mathrm{cm}^{2}$

B. PENETRATING DOSE AT - $\quad 1 \mathrm{~cm}, 5 \mathrm{~cm}$

\#18. A. THERMAL NEUTRON QUALITY FACTOR - 3

B. FAST NEUTRON QUALITY FACTOR - 10

C. TOTAL NEUTRON QUALITY FACTOR -

\#19. NONPENETRATING FILTER \#1-25mg $\mathrm{cm}^{2}$ OF PAPER

$\# 2$.

PENETRATING FILTER $\# 1-0.3 \mathrm{~cm}$ OF PLASTIC

\#2- $0.1 \mathrm{~cm} \mathrm{AI} \cdot 0.5 \mathrm{~cm}$ OF PLASTIC

$\# 3-0.15 \mathrm{~cm} \mathrm{Sn} \cdot \mathrm{Pb} \cdot 0.14 \mathrm{~cm}$ OF PLASTIC

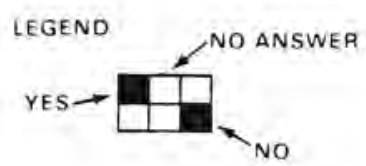


FACILITY - B

TYPE - NATIONAL LABORATORY

DOSIMETRY VOLUME - MEDIUM

DOSIMETRY SERVICE - INHOUSE

\section{SECTION I \\ $\frac{\text { DOSIMETRY DOCUMENTATION }}{1234}$ \\ $A$ B C O A B C

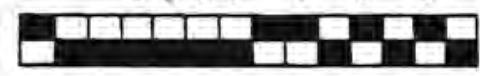

SECTION II

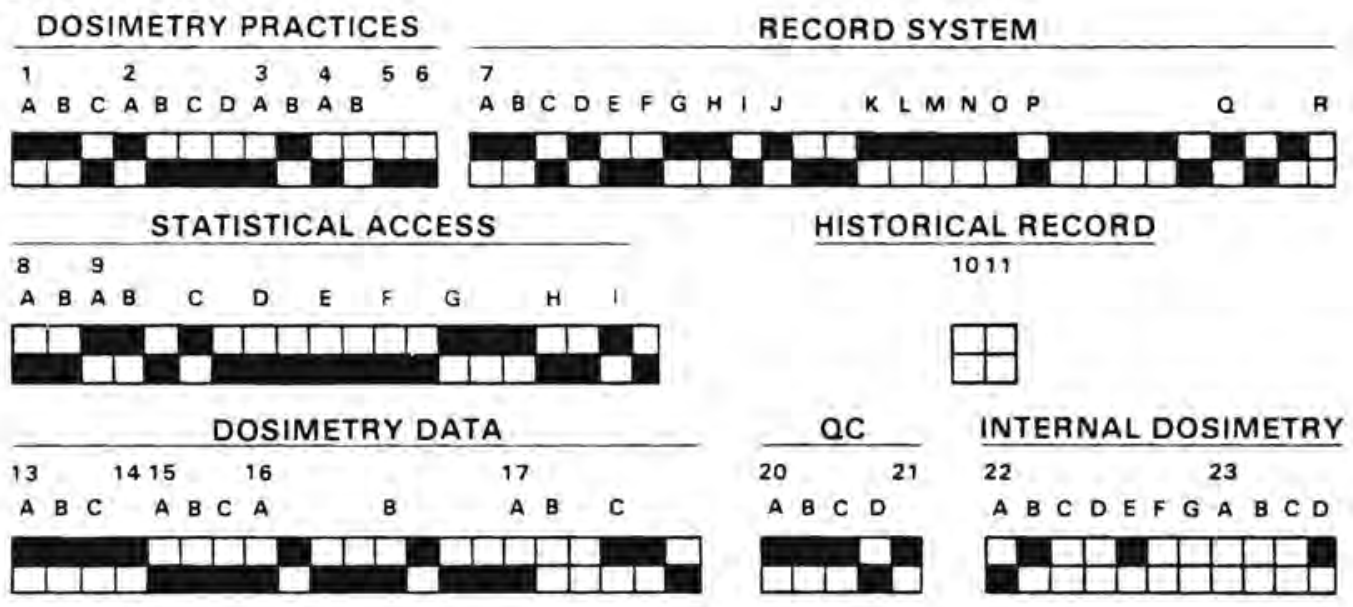

\section{SPECIFIC QUESTIONS AND ANSWERS}

\#12. NOT ANSWERED

\#17. A. NONPENETRATION DOSE AT - NOT STATED

B. PENETRATING DOSE AT - BASED ON Cd RATIO
\#18. A. THERMAL NEUTRON QUALITY FACTOR - NCRP $\# 38$ FLUX TO DOSE FACTORS
B. FAST NEUTRON QUALITY FACTOR -
C. TOTAL NEUTRON QUALITY FACTOR -

\#19. NONPENETRATING FILTER \#1.

$$
\# 2 \text {. }
$$

PENETRATING

$\begin{aligned} \text { FILTER } & \# 1-0.028 \mathrm{~cm} \mathrm{Cd} \\ & \# 2-\end{aligned}$

$\# 3$.

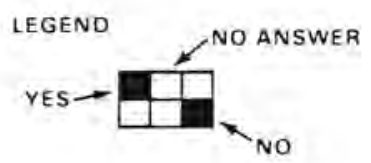


FACILITY - C

TYPE - NATIONAL LABORATORY

DOSIMETRY VOLUME - MEDIUM

DOSIMETRY SERVICE - INHOUSE

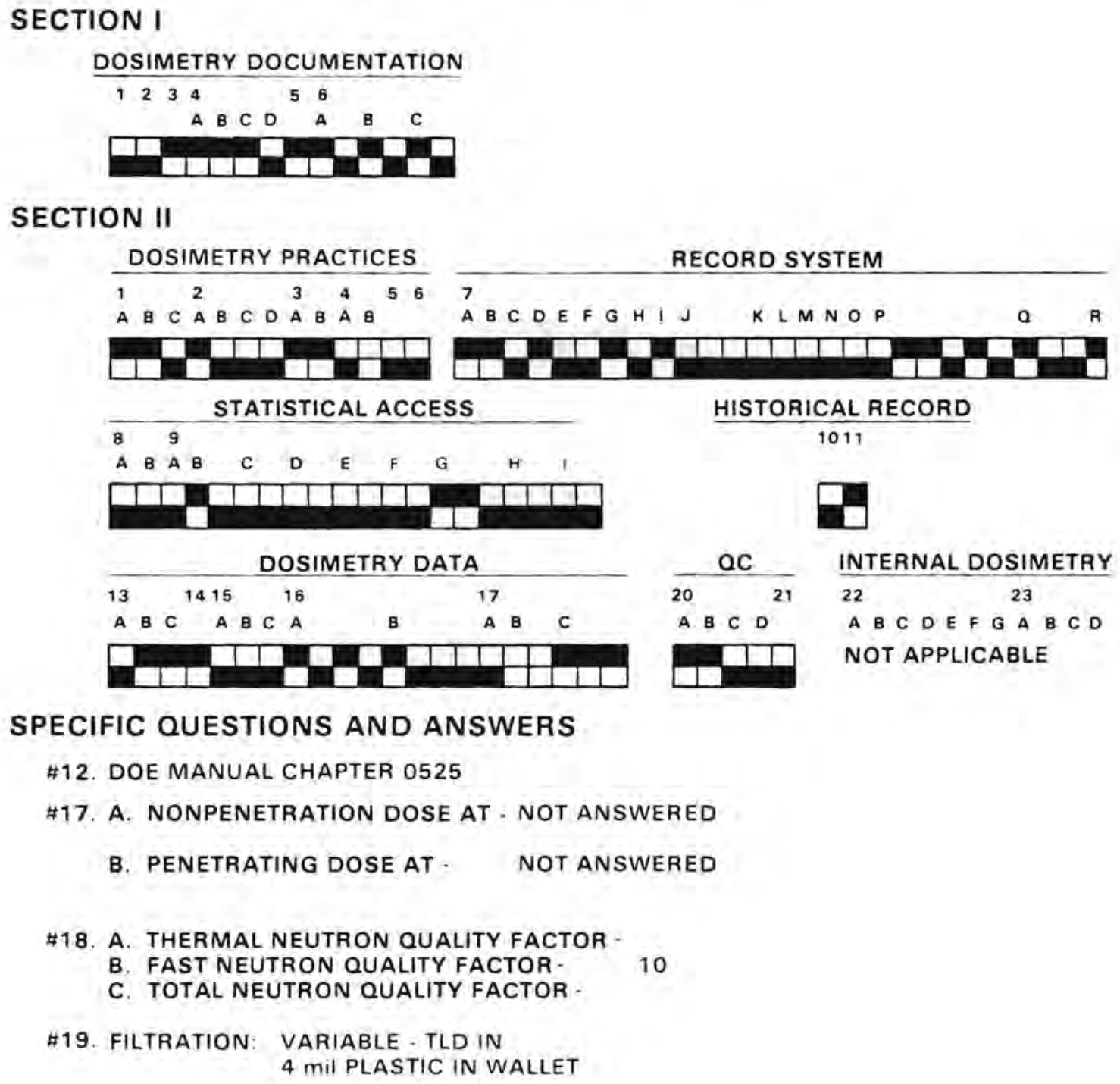

SPECIFIC QUESTIONS AND ANSWERS

\#12. DOE MANUAL CHAPTER 0525

\#17. A. NONPENETRATION DOSE AT - NOT ANSWERED

B. PENETRATING DOSE AT - NOT ANSWERED

\#18. A. THERMAL NEUTRON QUALITY FACTOR-

B. FAST NEUTRON QUALITY FACTOR - 10

C. TOTAL NEUTRON QUALITY FACTOR -

\#19. FILTRATION: VARIABLE - TLD IN

4 mil PLASTIC IN WALLET

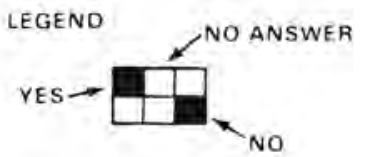


FACILITY - D

TYPE - NATIONAL LABORATORY

DOSIMETRY VOLUME - LARGE

DOSIMETRY SERVICE - INHOUSE

\author{
SECTION I \\ $\frac{\text { DOSIMETRY DOCUMENTATION }}{1234}$ \\ A B C D A B C

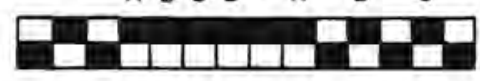

SECTION II

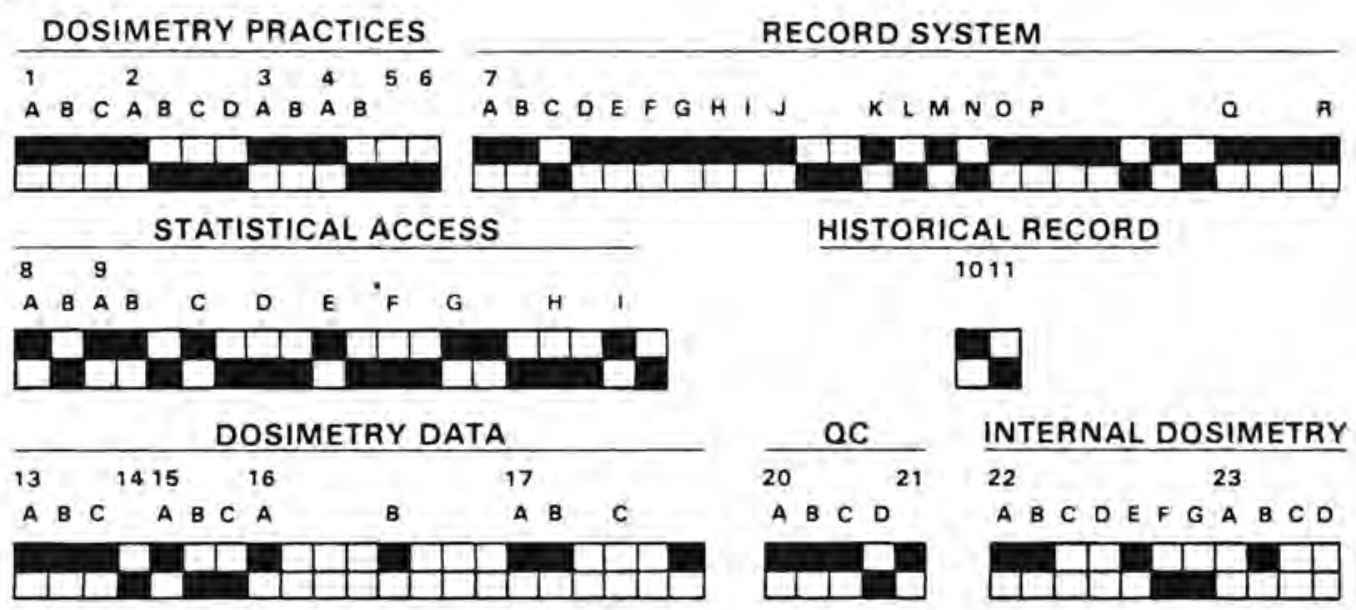

\title{
SPECIFIC QUESTIONS AND ANSWERS
}

\#12. DOE MANUAL CHAPTERS

\#17. A. NONPENETRATION DOSE AT $=7 \mathrm{mg} / \mathrm{cm}^{2}$

B. PENETRATING DOSE AT - $\quad 1 \mathrm{~cm}$

\#18. A. THERMAL NEUTRON QUALITY FACTOR -

B. FAST NEUTRON QUALITY FACTOR - 10

C. TOTAL NEUTRON QUALITY FACTOR -

\#19. NONPENETRATING FILTER \#1-40mg $\mathrm{cm}^{2}$ OF PLASTIC

$\# 2$.

PENETRATING FILTER \#1- $93 \mathrm{mg} \mathrm{cm}^{2}$ OF BRASS

$\# 2$ -

$\# 3$ -

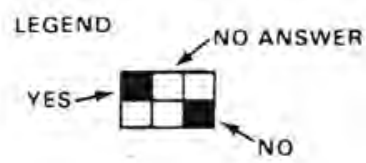


FACILITY - E

TYPE - NATIONAL LABORATORY

DOSIMETRY VOLUME - LARGE

DOSIMETRY SERVICE - INHOUSE

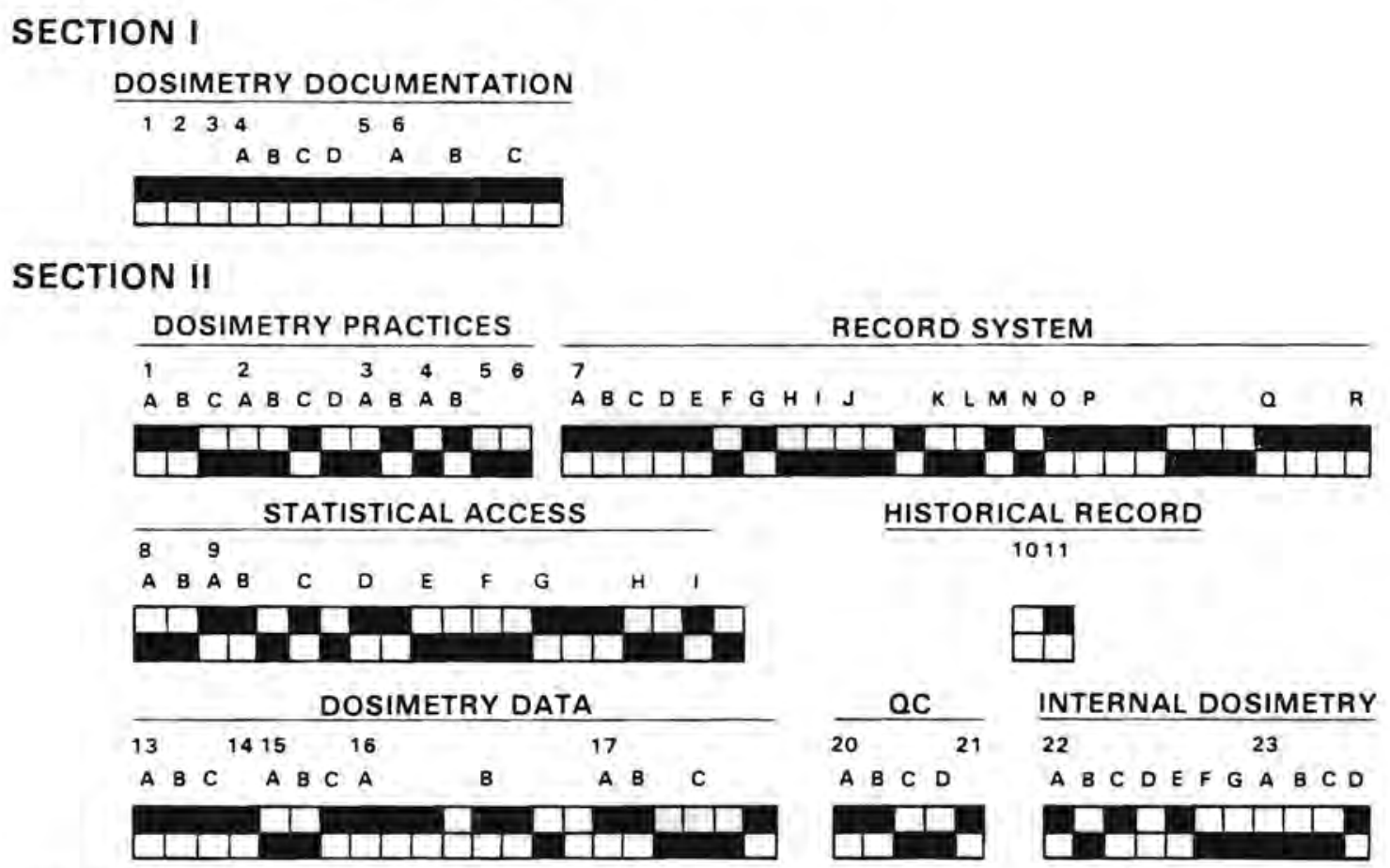

SPECIFIC QUESTIONS AND ANSWERS

\#12. DOE MANUAL CHAPTERS

\#17. A. NONPENETRATION DOSE AT $-7 \mathrm{mg} / \mathrm{cm}^{2}$

B. Penetrating dose at - $1 \mathrm{~cm}$

\#18. A. THERMAL NEUTRON QUALITY FACTOR - NCRP FLUX TO DOSE FACTORS

B. FAST NEUTRON QUALITY FACTOR. NCRP FLUX TO DOSE FACTORS

C. TOTAL NEUTRON QUALITY FACTOR .

\#19. NONPENETRATING FILTER \#1. $50 \mathrm{mg} / \mathrm{cm}^{2}$ OF PLASTIC

$\# 2$ - $150 \mathrm{mg} . \mathrm{cm}^{2}$ OF PLASTIC

PENETRATING FILTER $\# 1-1000 \mathrm{mg} \mathrm{cm}^{2}$ OF Al-PLASTIC

$\# 2$.

$\# 3$.

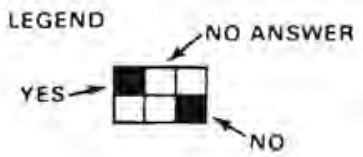

B. 5 
FACILITY - F

TYPE - NATIONAL LABORATORY

DOSIMETRY VOLUME - MEDIUM

DOSIMETRY SERVICE - INHOUSE

\section{SECTION I}

$\frac{\text { DOSIMETRY DOCUMENTATION }}{12334}$

$A$ B C D A B C

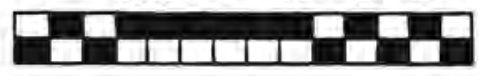

SECTION II

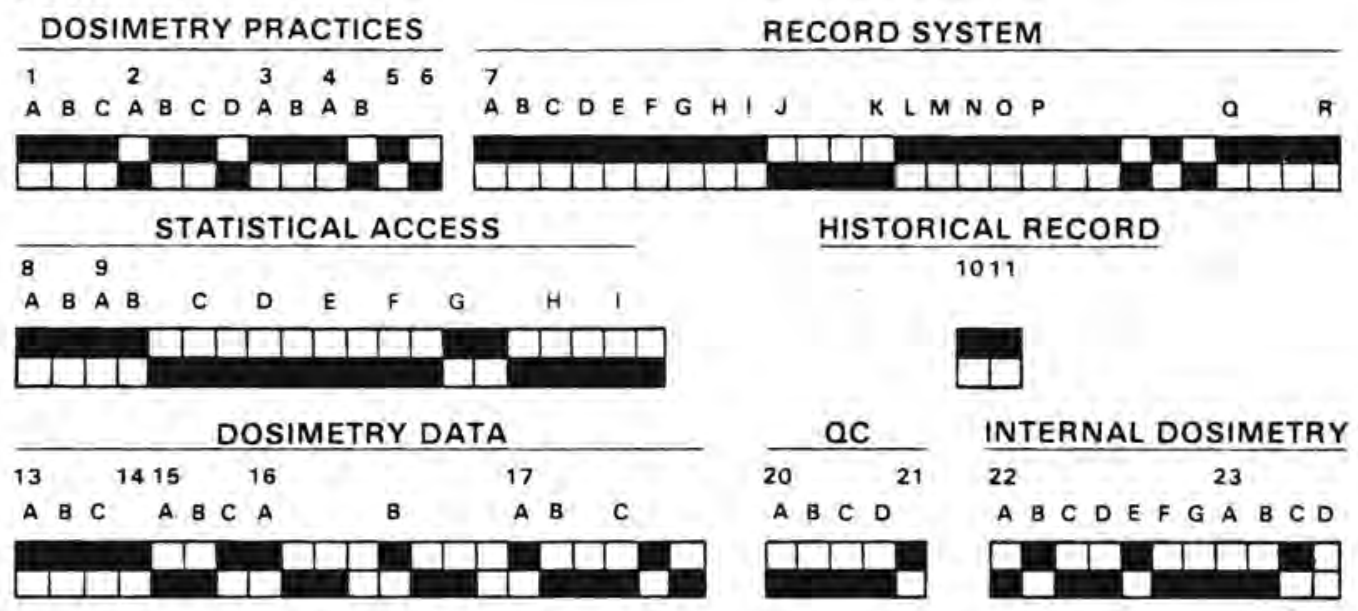

SPECIFIC QUESTIONS AND ANSWERS

\#12. DOE MANUAL CHAPTER 0525.

\#17. A. NONPENETRATION DOSE AT $-7 \mathrm{mg} / \mathrm{cm}^{2}$

B. PENETRATING DOSE AT - $270 \mathrm{mg} / \mathrm{cm}^{2}$

\#18. A. THERMAL NEUTRON QUALITY FACTOR -
B. FAST NEUTRON QUALITY FACTOR.

C. TOTAL NEUTRON QUALITY FACTOR-

\#19. NONPENETRATING FILTER \#1,7mg/ $\mathrm{cm}^{2}$ PLASTIC

$\# 2$.

PENETRATING FILTER \#1- $0.1 \mathrm{~cm} \mathrm{AI}$

$\# 2$.

\#3

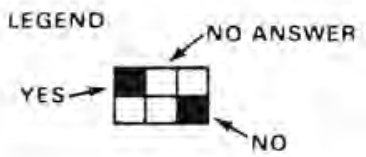


FACILITY - G

TYPE - NATIONAL LABORATORY

DOSIMETRY VOLUME - LARGE

DOSIMETRY SERVICE - INHOUSE

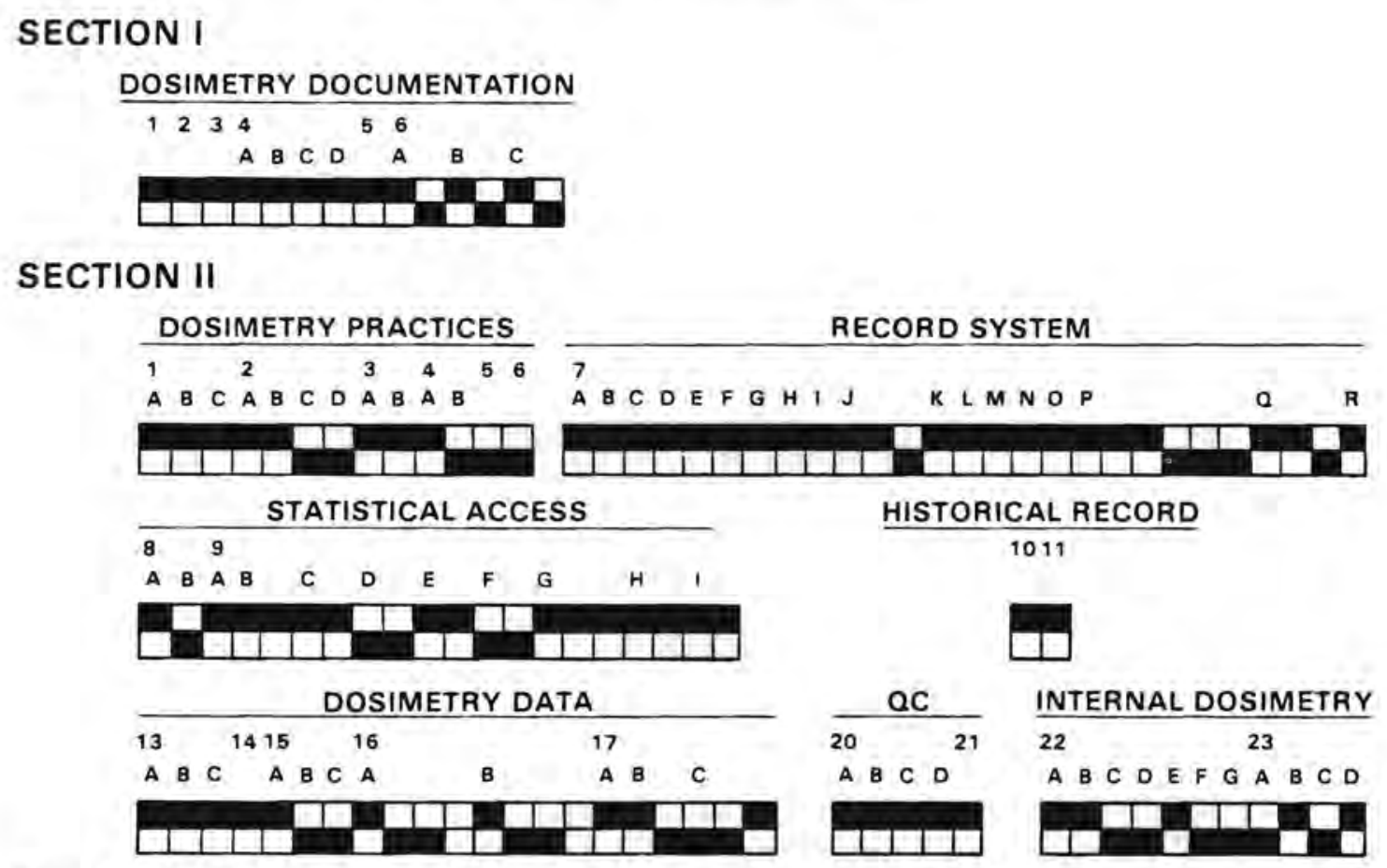

SPECIFIC QUESTIONS AND ANSWERS

\#12. DOE MANUAL APPENDIX 0230C-8

\#17. A. NONPENETRATION DOSE AT $-7 \mathrm{mg} / \mathrm{cm}^{2}$

B. PENETRATING DOSE AT - $1 \mathrm{~cm}$

\#18. A. THERMAL NEUTRON QUALITY FACTOR - 3

B. FAST NEUTRON QUALITY FACTOR - 10

C. TOTAL NEUTRON QUALITY FACTOR - 10

\#19. NONPENETRATING FILTER \#1-5.6 $\mathrm{mg} / \mathrm{cm}^{2}$ PLASTIC $\# 2$.

PENETRATING FILTER \#1-0.17cm AI

$\# 2$ -

$\# 3$.

LEGEND NOANSWER 
FACILITY - H

TYPE - NATIONAL LABORATORY

DOSIMETRY VOLUME - MEDIUM

DOSIMETRY SERVICE - COMMERCIAL

\section{SECTION I \\ DOSIMETRY DOCUMENTATION

$\begin{array}{lllllll}1234 & 6 & & \\ A B C D & A & B & C\end{array}$

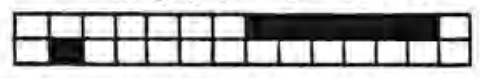

SECTION II

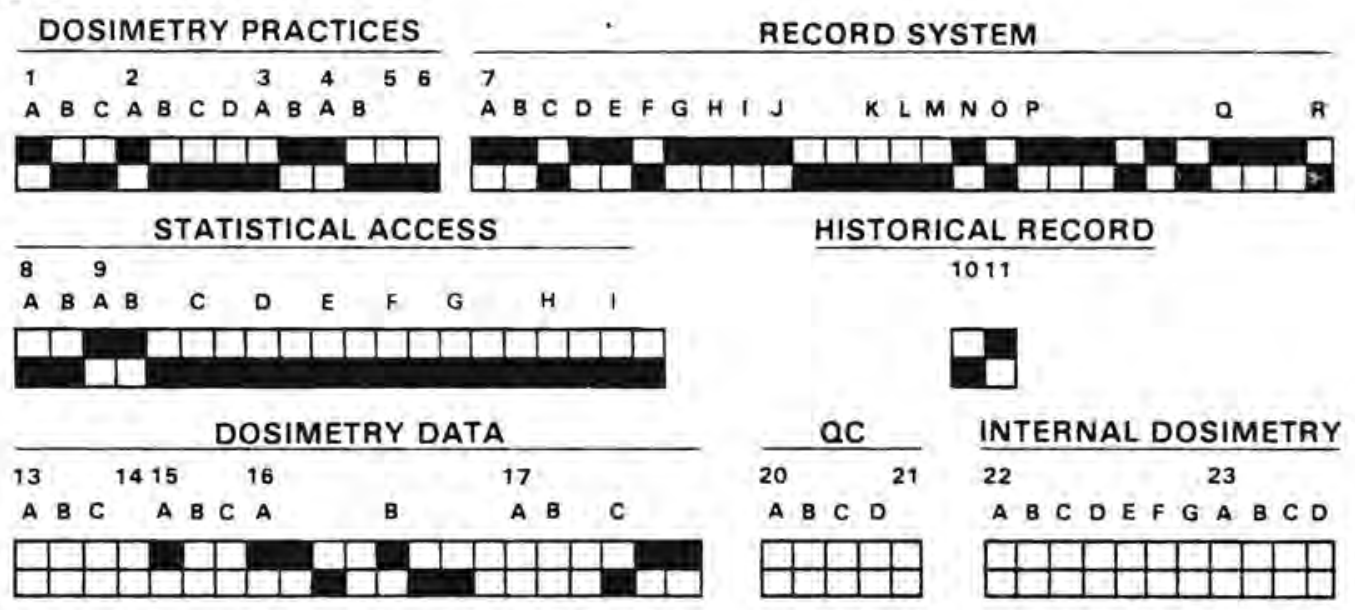

SPECIFIC QUESTIONS AND ANSWERS

\#12. DOE MANUAL 0231 ANNEX C

\#17. A. NONPENETRATION DOSE AT -

B. PENETRATING DOSE AT -

\#18. A. THERMAL NEUTRON QUALITY FACTOR -

B. FAST NEUTRON QUALITY FACTOR - 10

C. TOTAL NEUTRON QUALITY FACTOR -

\#19. NONPENETRATING FILTER \#1 - NOT ANSWERED

$\# 2$.

PENETRATING FILTER $\begin{aligned} & \# 1 \text { - NOT ANSWERED } \\ & \# 2- \\ & \# 3 .\end{aligned}$

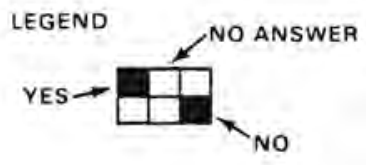


FACILITY - I

TYPE - NATIONAL LABORATORY

DOSIMETRY VOLUME - LARGE

DOSIMETRY SERVICE - INHOUSE

\author{
SECTION I \\ DOSIMETRY DOCUMENTATION \\ $1234 \quad 56$ \\ A B C D A B C

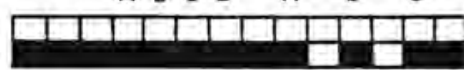

SECTION II

DOSIMETRY PRACTICES

$\begin{array}{llllll}1 & 2 & 3 & 4 & 56 & 7\end{array}$

A B C A BC D A B A B

A B C DEFGH I KLMNOP

RECORD SYSTEM
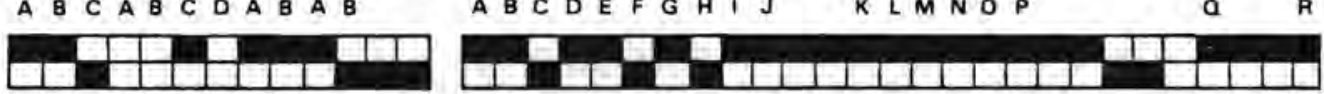

STATISTICAL ACCESS

89

STATISTICAL ACCESS

HISTORICAL RECORD

A B A B C D E F G

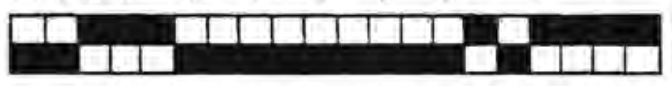

1011

DOSIMETRY DATA
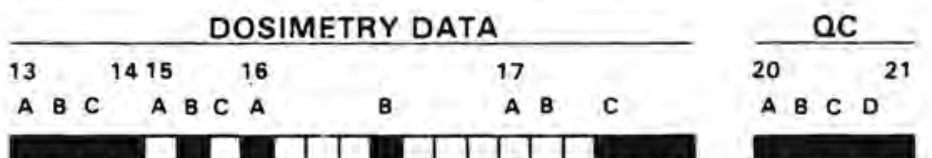

$\square$
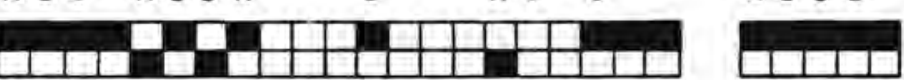

$\frac{\text { INTERNAL DOSIMETRY }}{22}$

A B C DEFGABCD

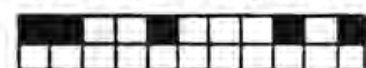

\title{
SPECIFIC QUESTIONS AND ANSWERS
}

\#12. DOE APPENDIX 0230

\#17. A. NONPENETRATION DOSE AT $=32 \mathrm{mg} / \mathrm{cm}^{2}$

B. PENETRATING DOSE AT - $\quad 1400 \mathrm{mg} / \mathrm{cm}^{2}$

\#18. A. THERMAL NEUTRON QUALITY FACTOR - FACILITY SPECIFIC FACTORS

B. FAST NEUTRON QUALITY FACTOR - FACILITY SPECIFIC FACTORS

C. TOTAL NEUTRON QUALITY FACTOR - FACILITYY SPECIFIC FACTORS

\#19. NONPENETRATING filteR \#1-32mg/ $\mathrm{cm}^{2}$ CELlulose ACETATE

$\# 2$.

PENETRATING FILTER $\# 1-1400 \mathrm{mg} / \mathrm{cm}^{2}$ PLASTIC

$\# 2$ -

$\# 3$ -

LEGEND NO ANSWER 
FACILITY - J

TYPE - NATIONAL LABORATORY

DOSIMETRY VOLUME - LARGE

DOSIMETRY SERVICE - INHOUSE.

\section{SECTION I \\ $\frac{\text { DOSIMETRY DOCUMENTATION }}{1234}$ \\ $A B C D$ A B C

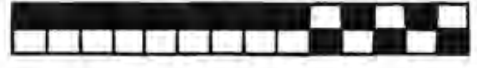

SECTION II

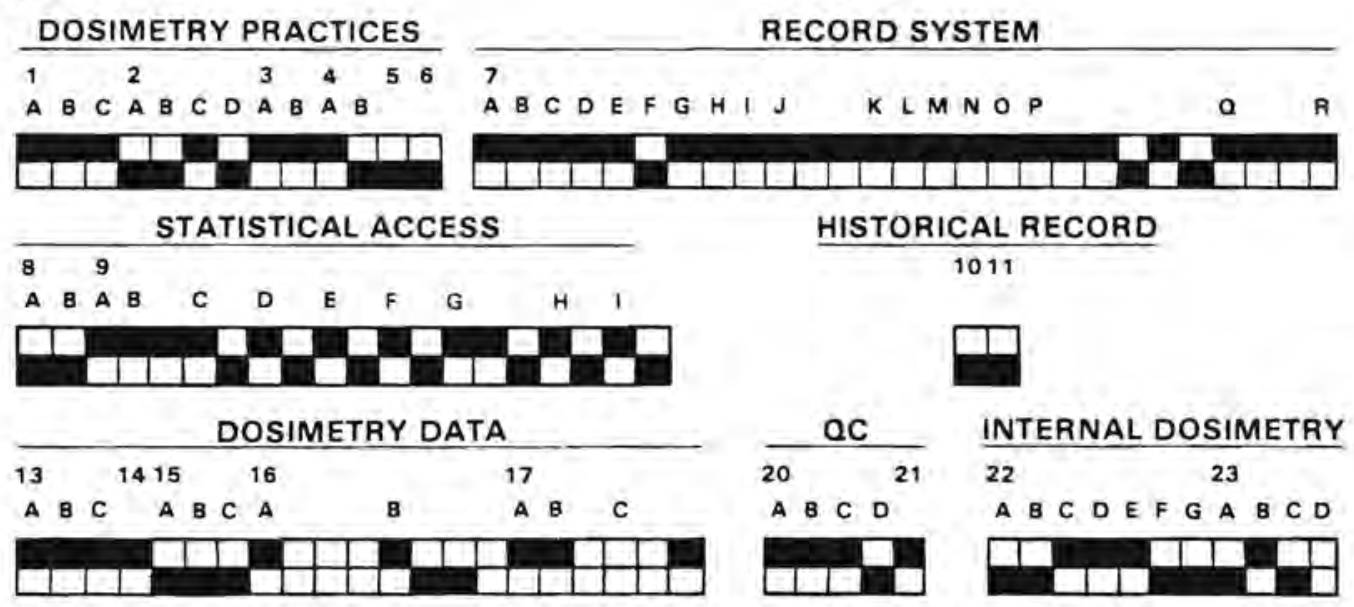

\section{SPECIFIC QUESTIONS AND ANSWERS}

\#12. DOE MANUAL CHAPTERS

\#17. A. NONPENETRATION DOSE AT. $7 \mathrm{mg} / \mathrm{cm}^{2}$

B. PENETRATING dose at - $\quad 1 \mathrm{~cm}$

\#18. A. THERMAL NEUTRON QUALITY FACTOR -

B. FAST NEUTRON QUALITY FACTOR.

C. TOTAL NEUTRON QUALITY FACTOR - MC 0524 PART 4

\#19. NONPENETRATING FILTER \#1- $7 \mathrm{mg} / \mathrm{cm}^{2}$ PLASTIC

$\# 2$.

PENETRATING FILTER $\# 1-0.0025 \mathrm{~cm}$ BRASS

$\# 2$ -

\#3.

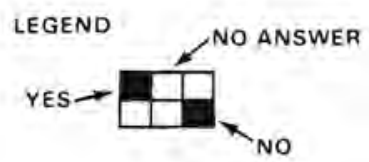


FACILITY - K

TYPE - NATIONAL LABORATORY

DOSIMETRY VOLUME - LARGE

DOSIMETRY SERVICE - INHOUSE

\author{
SECTION I \\ DOSIMETRY DOCUMENTATION \\ A B C D A B C

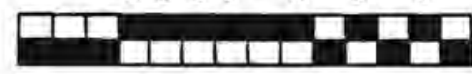

SECTION II

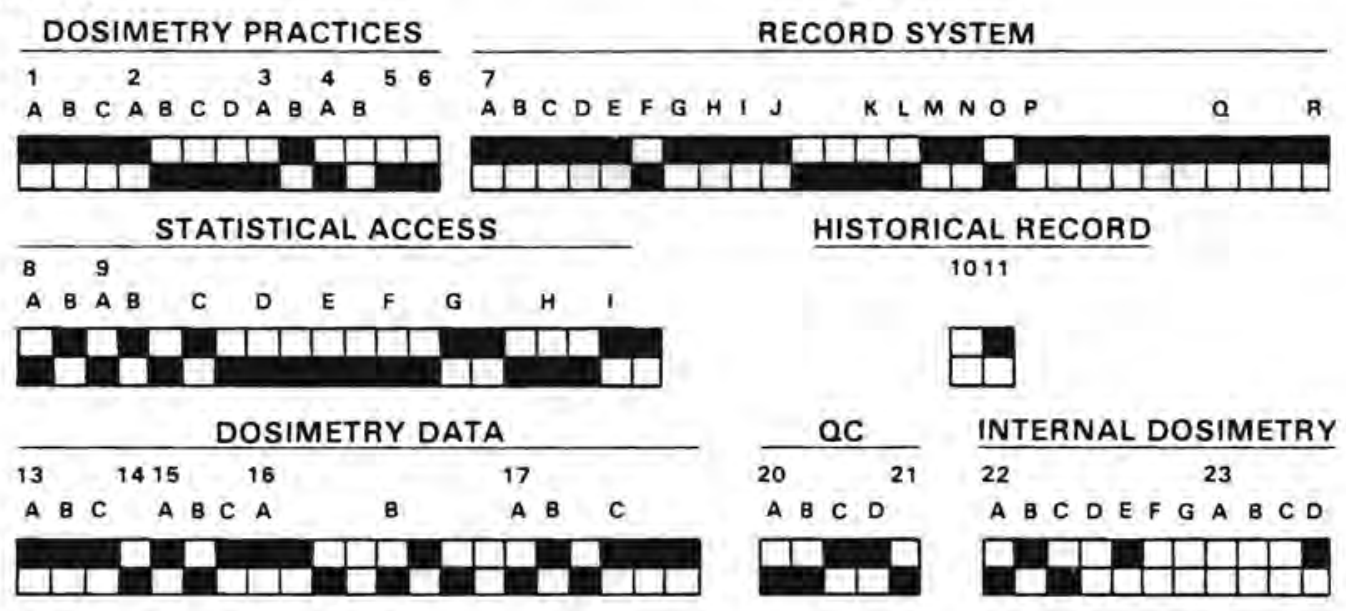

\title{
SPECIFIC QUESTIONS AND ANSWERS
}

\#12. DOE MANUAL CHAPTER 0525

\#17. A. NONPENETRATION DOSE AT $-23 \mathrm{mg} / \mathrm{cm}^{2}$

B. PENETRATING DOSE AT - $1 \mathrm{~cm}$

\#18. A. THERMAL NEUTRON QUALITY FACTOR - 1-3

B. FAST NEUTRON QUALITY FACTOR-

C. TOTAL NEUTRON QUALITY FACTOR -

\#19. NONPENETRATING FILTER \#1-23mg $/ \mathrm{cm}^{2}$

\#2.

PENETRATING

FILTER \#1-1400mg $/ \mathrm{cm}^{2} \mathrm{Cd}+\mathrm{SS}$

$\# 2-462 \mathrm{mg} / \mathrm{cm}^{2}$ Al

\#3.

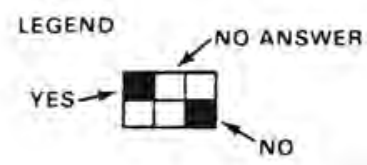


FACILITY - L (OLD SYSTEM - FILM)

TYPE - NATIONAL LABORATORY

DOSIMETRY VOLUME - LARGE DOSIMETRY SERVICE - INHOUSE

\author{
SECTION I \\ DOSIMETRY DOCUMENTATION \\ $\begin{array}{llllll}1234 & 56\end{array}$ \\ $A$ B C D A B C

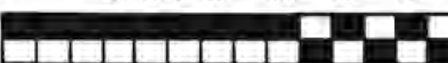

SECTION II

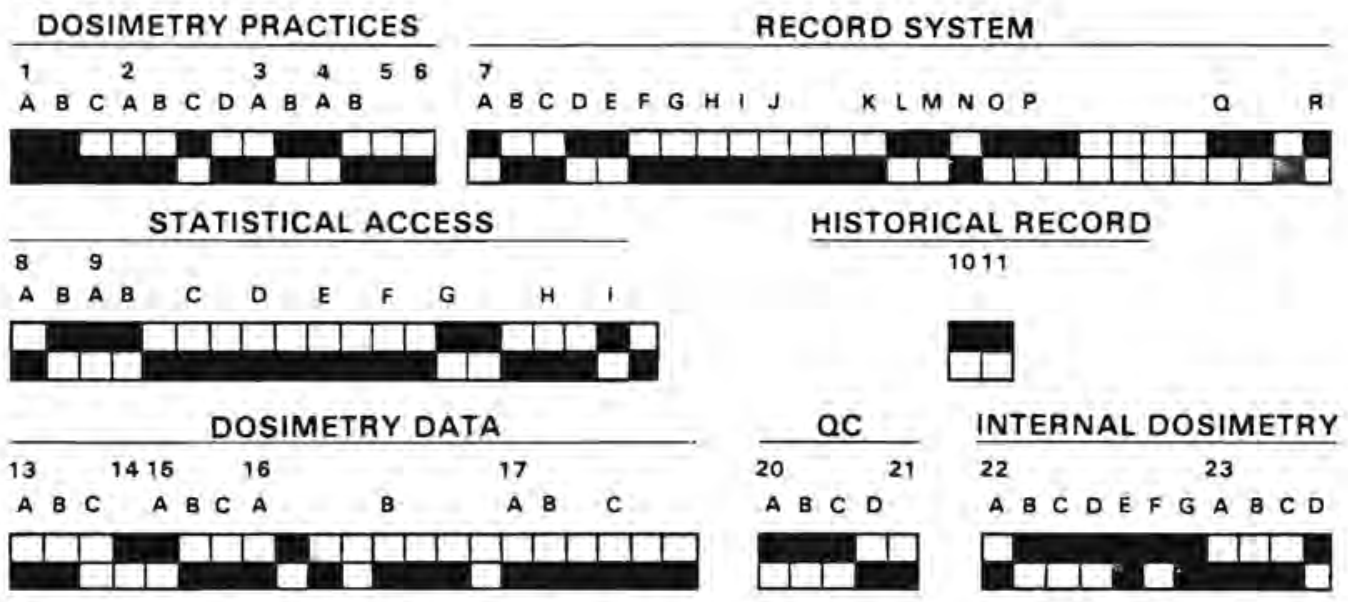

\title{
SPECIFIC QUESTIONS AND ANSWERS
}

\#12. DOE MANUAL CHAPTERS

\#17. A. NONPENETRATION DOSE AT - SURFACE DOSE RELATIVE TO

B. PENETRATING DOSE AT DEPLETED URANIUM

GAMMA DOSE RELATIVE TO ${ }^{60} \mathrm{Co}$

\#18. A. THERMAL NEUTRON QUAI.ITY FACTOR -

B. FAST NEUTRON QUALITY FACTOR -

C. TOTAL NEUTRON QUALITY FACTOR -

\#19. NONPENETRATING FILTER \#1-80 $\mathrm{mg} / \mathrm{cm}^{2}$ PLASTIC + PAPER

$\# 2$.

PENETRATING

FILTER $\# 1-0.076 \mathrm{~cm} \mathrm{Cd}$

$\# 2-0.013 \mathrm{~cm} \mathrm{Au}$

$\# 3-0.081 \mathrm{~cm}$ PLASTIC - PAPER

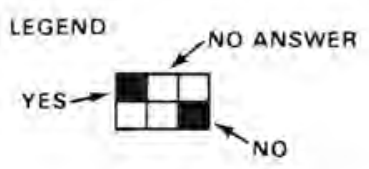




\title{
FACILITY - L (NEW SYSTEM) \\ TYPE - NATIONAL LABORABORY \\ DOSIMETRY VOLUME - LARGE DOSIMETRY SERVICE - INHOUSE
}

\author{
SECTION I \\ DOSIMETRY DOCUMENTATION

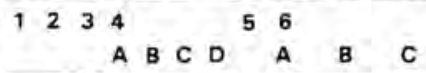

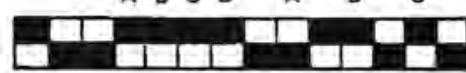

SECTION II

DOSIMETRY PRACTICES $\begin{array}{llllll}1 & 2 & 3 & 4 & 56\end{array}$

A B C AB C DABAB

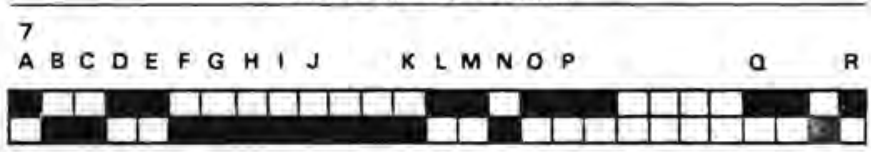

STATISTICAL ACCESS

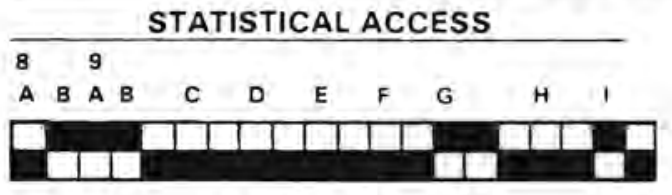

HISTORICAL RECORD

1011

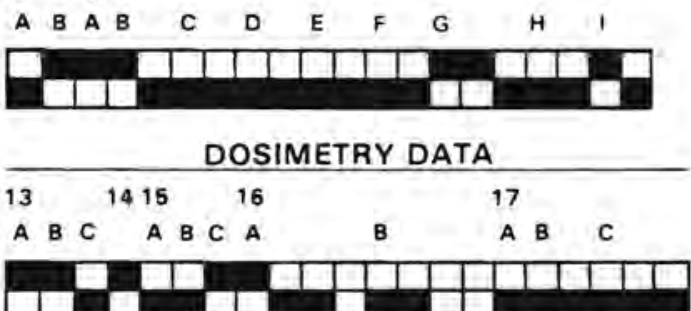

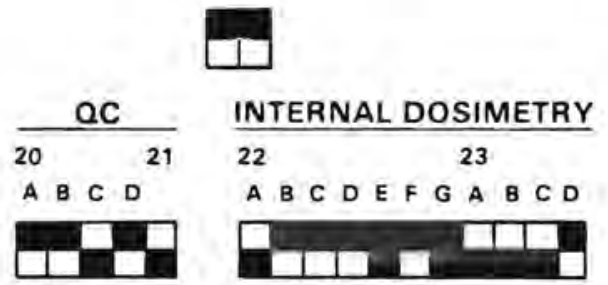

SPECIFIC QUESTIONS AND ANSWERS

\#12. DOE MANUAL CHAPTERS

\#17. A. NONPENETRATION DOSE AT - SURFACE DOSE RELATIVE TO DEPLETED URANIUM

B. penetrating dose at - gamma dose relative to $6^{\circ} \mathrm{Co}$

\#18. A. THERMAL NEUTRON QUALITY FACTOR -

B. FAST NEUTRON QUALITY FACTOR -

C. TOTAL NEUTRON QUALITY FACTOR.

\#19. NONPENETRATING FILTER \#1-70mg $/ \mathrm{cm}^{2}$ PLASTIC + PAPER

$\# 2$.

PENETRATING FILTER $\# 1-700 \mathrm{mg} / \mathrm{cm}^{2}$, FIBERGLASS, PLASTIC, PAPER AND AI $\# 2$ $\# 3$ -

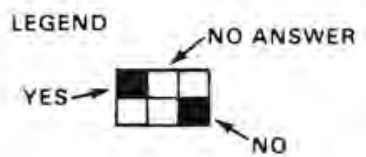


FACILITY - M

TYPE - NATIONAL LABORATORY

DOSIMETRY VOLUME - LARGE

DOSIMETRY SERVICE - INHOUSE

\section{SECTION I \\ DOSIMETRY DOCUMENTATION \\ $1234 \quad 56$

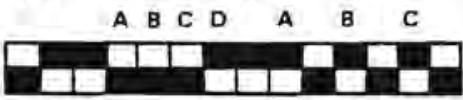

SECTION II

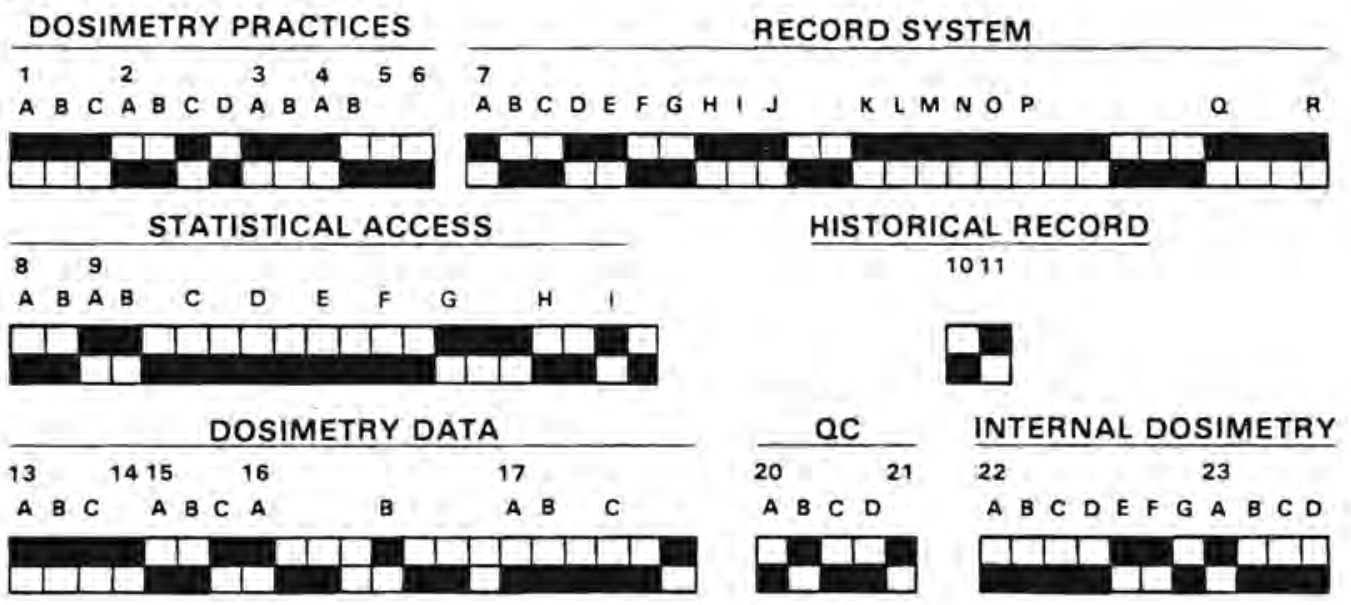

SPECIFIC QUESTIONS AND ANSWERS

\#12. MANUAL CHAPTER 0524

\#17. A. NONPENETRATION DOSE AT $-15 \mathrm{mg} / \mathrm{cm}^{2}$

B. PENETRATING DOSE AT $\quad 2 \mathrm{~cm}$

\#18. A. THERMAL NEUTRON QUALITY FACTOR -

B. FAST NEUTRON QUALITY FACTOR -

C. TOTAL NEUTRON QUALITY FACTOR - 2-11

\#19. NONPENETRATING FILTER \#1-15 mg $\mathrm{cm}^{2}$ TEFLON

\#2.

PENETRATING FILTER \#1-0.127cm OF AI

$\# 2$ -

\#3-

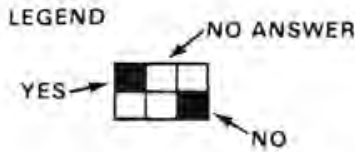


FACILITY - N

TYPE - NATIONAL LABORATORY

DOSIMETRY VOLUME - MEDIUM

DOSIMETRY SERVICE - FACILITY P

\author{
SECTION I \\ DOSIMETRY DOCUMENTATION

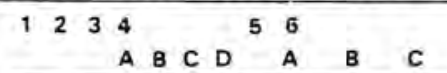

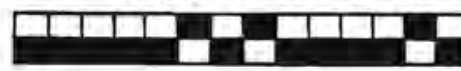

SECTION II

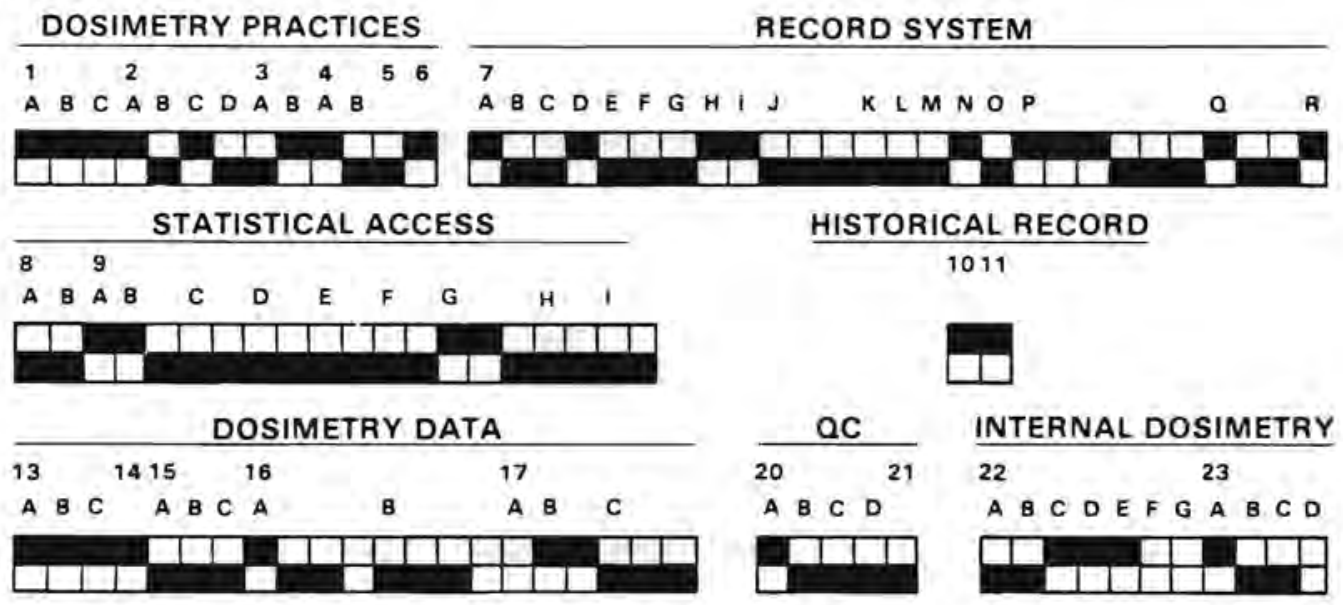

SPECIFIC QUESTIONS AND ANSWERS

\#12. DOE MANUAL CHAPTERS

\#17. A. NONPENETRATION DOSE AT - UNKNOWN

B. PENETRATING DOSE AT - 1 AND $5 \mathrm{~cm}$

\#18. A. THERMAL NEUTRON QUALITY FACTOR -

B. FAST NEUTRON QUALITY FACTOR -

C. TOTAL NEUTRON QUALITY FACTOR -

\#19. NONPENETRATING FILTER $\# 1-12 \mathrm{mg} / \mathrm{cm}^{2}$ AI FOIL

\#2.

PENETRATING FILTER \#1.

$\# 2$ -

\#3-

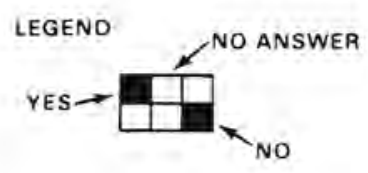


FACILITY - O

TYPE - NATIONAL LABORATORY

DOSIMETRY VOLUME - LARGE

DOSIMETRY SERVICE - INHOUSE

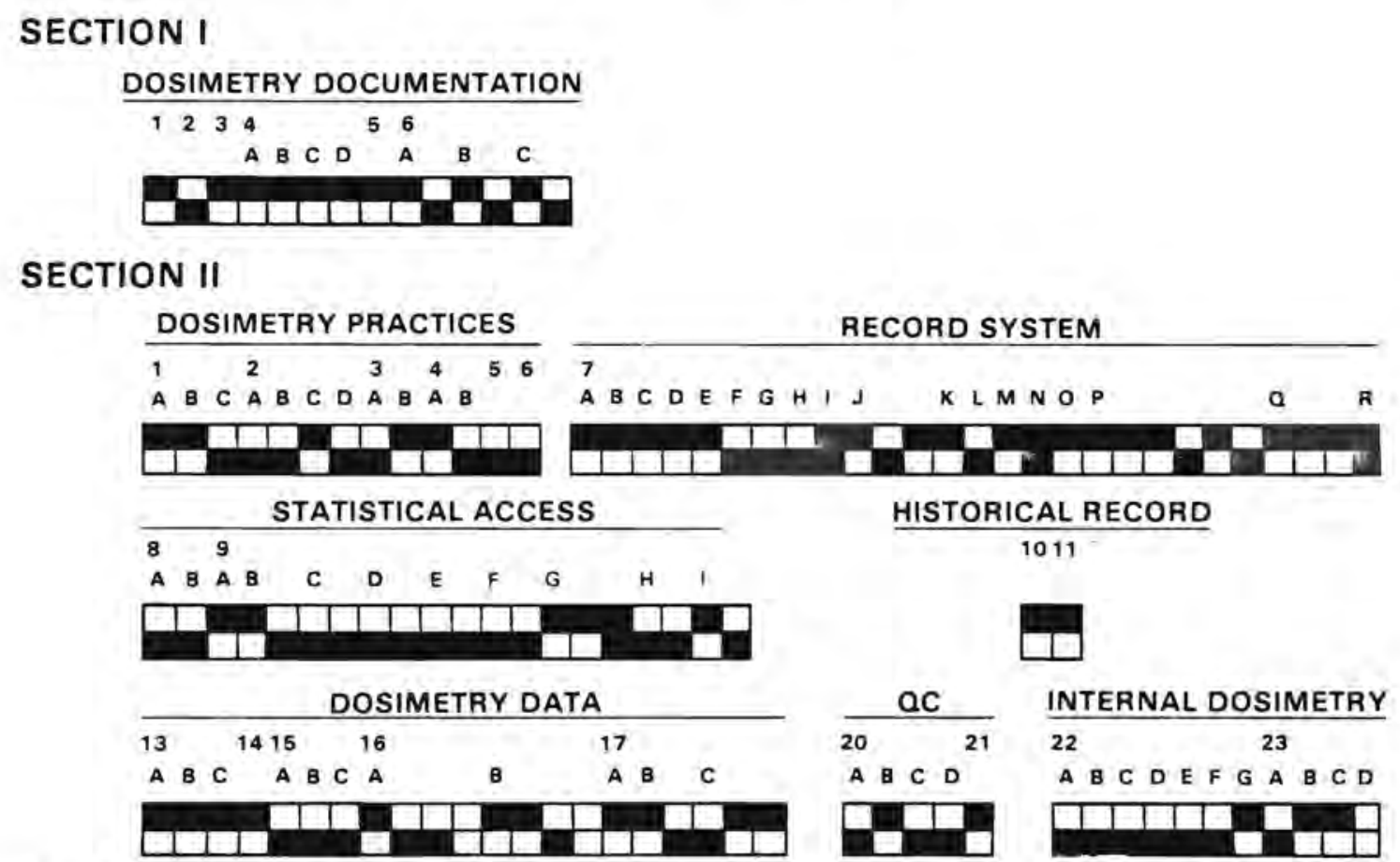

SPECIFIC QUESTIONS AND ANSWERS

\#12. DOE MANUAL CHAPTERS

\#17. A. NONPENETRATION DOSE AT $-7 \mathrm{mg} / \mathrm{cm}^{2}$

B. penetrating dose at - $1 \mathrm{~cm}$

\#18. A. THERMAL NEUTRON QUALITY FACTOR - NCRP \#38

B. FAST NEUTRON QUALITY FACTOR - NCRP \#38

C. TOTAL NEUTRON QUALITY FACTOR -

NCRP $\# 38$,

\#19. NONPENETRATING FILTER $\# 1-60 \mathrm{mg} \mathrm{cm}^{2}$ PLASTIC $\# 2$.

PENETRATING FILTER $\# 1.90 \mathrm{mg} / \mathrm{cm}^{2}$ COPPER $\# 2$. $250 \mathrm{mg} \mathrm{cm}^{2}$ PLASTIC $\# 3$.

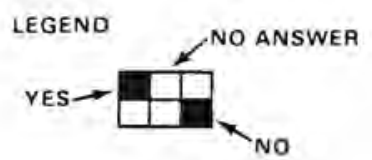


FACILITY - P

TYPE - NATIONAL LABORATORY

DOSIMETRY VOLUME - LARGE

DOSIMETRY SERVICE - INHOUSE

\author{
SECTION I \\ DOSIMETRY DOCUMENTATION

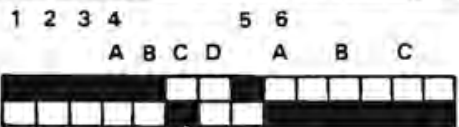

SECTION II

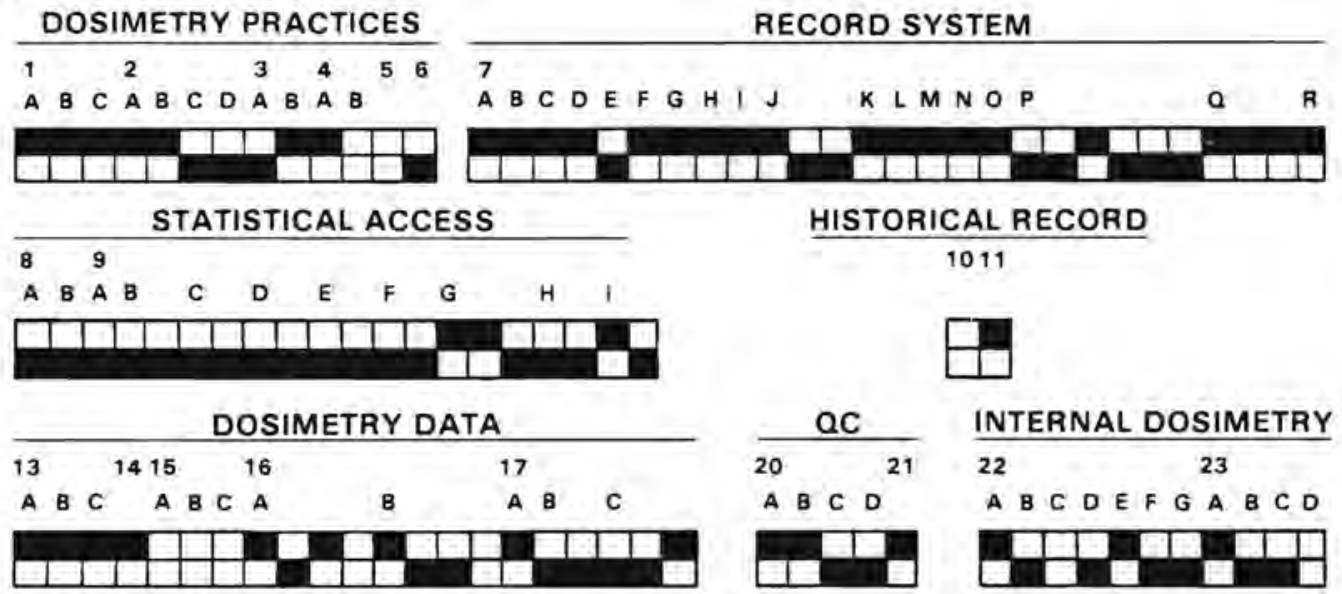

SPECIFIC QUESTIONS AND ANSWERS

\#12. RECORDS RETAINED INDEFINITELY

\#17. A. NONPENETRATION DOSE AT $-7 \mathrm{mg} / \mathrm{cm}^{2}$

B. PENETRATING DOSE AT - $\quad 1 \mathrm{~cm}$

\#18. A. THERMAL NEUTRON QUALITY FACTOR - 3"/9" MEASUREMENTS

B. FAST NEUTRON QUALITY FACTOR - 3"/9" MEASUREMENTS

C. TOTAL NEUTRON QUALITY FACTOR.

\#19. NONPENETRATING FILTER \#1-4.5mg $/ \mathrm{cm}^{2}$ MYLAR

\#2.

PENETRATING FILTER \#1-0.2 cm OF AI

\#2 -

\#3-

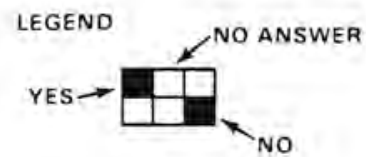


FACILITY - Q

TYPE - NATIONAL LABORATORY

DOSIMETRY VOLUME - LARGE

DOSIMETRY SERVICE - INHOUSE

\section{SECTION I \\ $\frac{\text { DOSIMETRY DOCUMENTATION }}{1234}$ \\ A B C D A B B C

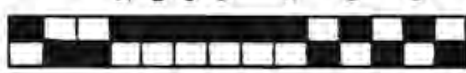

SECTION II

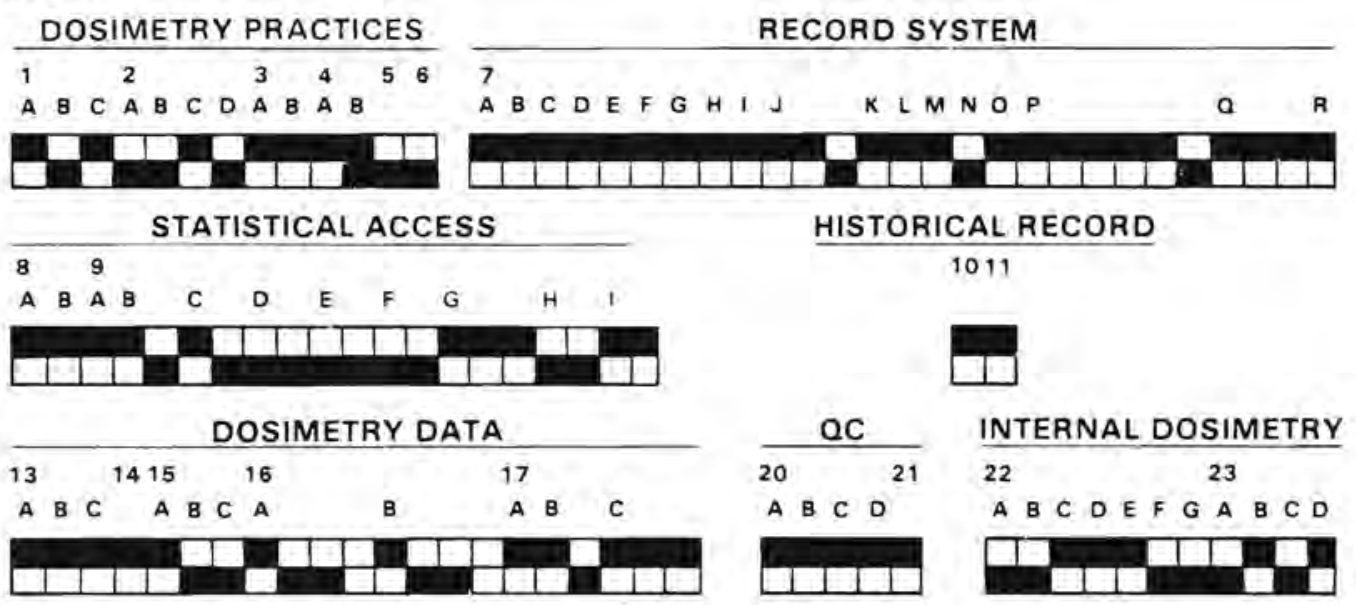

\section{SPECIFIC QUESTIONS AND ANSWERS}

\#12. DOE MANUAL CHAPTERS ANSI 13.6. PRIVACY ACT

\#17. A. NONPENETRATION DOSE AT $-7 \mathrm{mg} \mathrm{cm}^{2}$

B. PENETRATING DOSE AT . $\quad 1 \mathrm{~cm}$

\#18. A. THERMAL NEUTRON QUALITY FACTOR - 3

B. FAST NEUTRON QUALITY FACTOR - 10

C. TOTAL NEUTRON QUALITY FACTOR - NOT APPLICABLE

\#19. NONPENETRATING FILTER \#1- $80 \mathrm{mg} \mathrm{cm}^{2}$ PLASTIC $\# 2$.

PENETRATING

FILTER \#1- $80 \mathrm{mg} \mathrm{cm}^{2}$ PLASTIC

\#2- $93 \mathrm{mg} \mathrm{cm} \mathrm{cm}^{7}$ PLASTIC

$\# 3.173 \mathrm{mg} \mathrm{cm}^{2} \mathrm{Al}$

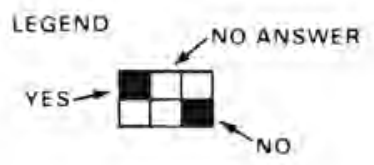


FACILITY - R

TYPE - NATIONAL LABORATORY

DOSIMETRY VOLUME - LARGE

DOSIMETRY SERVICE - INHOUSE

\section{SECTION 1 \\ DOSIMETRY DOCUMENTATION

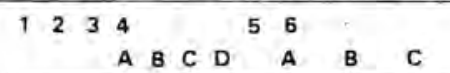

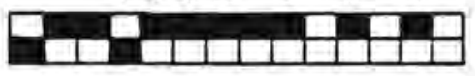

SECTION II

DOSIMETRY PRACTICES $\begin{array}{lllllll}1 & 2 & 3 & 4 & 5 & 6 & 7\end{array}$

A B C ABCDAB A B

RECORD SYSTEM

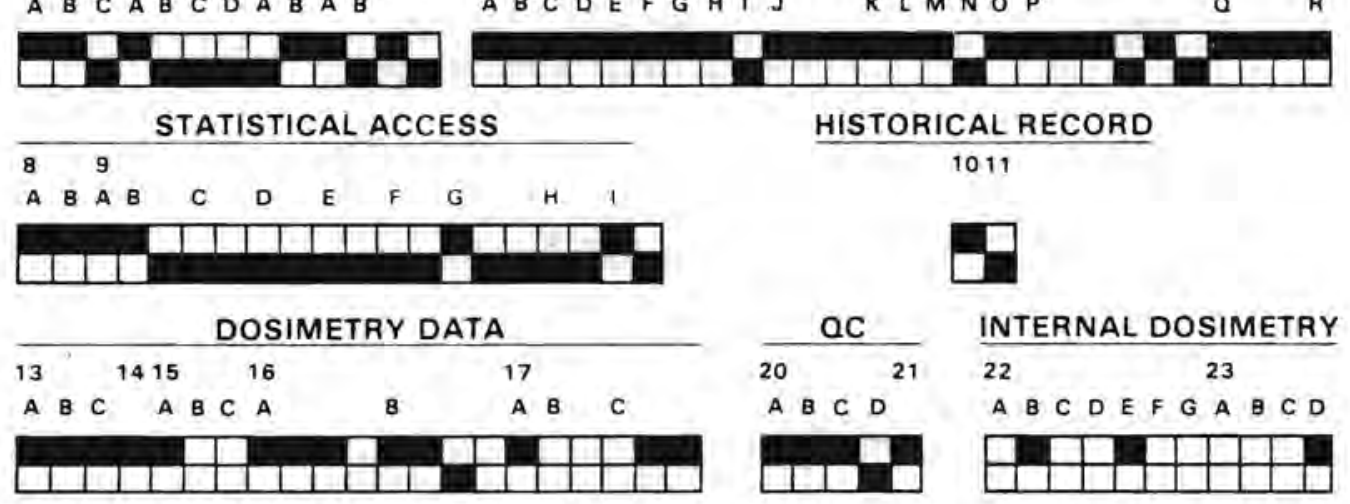

\section{SPECIFIC QUESTIONS AND ANSWERS}

\#12. DOE MANUAL CHAPTERS

\#17. A. NONPENETRATION DOSE AT $-7 \mathrm{mg} \mathrm{cm}^{2}$

B. PENETRATING DOSEAT - SURFACE OF SKIN

\#18. A. THERMAL NEUTRON QUALITY FACTOR-
B. FAST NEUTRON QUALITY FACTOR-
C. TOTAL NEUTRON QUALITY FACTOR -

\#19. NONPENETRATING FILTER $\# 1-20-30 \mathrm{mg} \mathrm{cm}^{2}$ PLASTIC

$\# 2$ - $130 \mathrm{mg} \mathrm{cm} \mathrm{cm}^{2} \mathrm{Al}$

\#3 $130 \mathrm{mg} \mathrm{cm} \mathrm{cm}^{2} \mathrm{Ag}$

PENETRATING

FILTER $\# 1-0.1 \mathrm{~cm}$ OF Cd

$\# 2-0.02 \mathrm{~cm}$ OF PLASTIC

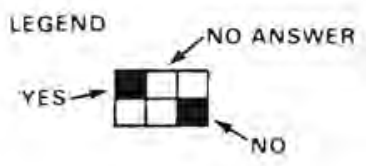


FACILITY - S

TYPE - NATIONAL LABORATORY

DOSIMETRY VOLUME - LARGE

DOSIMETRY SERVICE - INHOUSE

\section{SECTION I}

DOSIMETRY DOCUMENTATION

$1234 \quad 56$

A B C D A B C

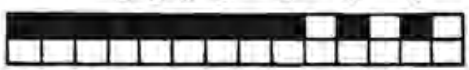

SECTION II

DOSIMETRY PRACTICES

$\begin{array}{lllllll}1 & 2 & 3 & 4 & 5 & 6 & 7\end{array}$

ABCABCDABAB ABCDEFGHIJ KLMNOP

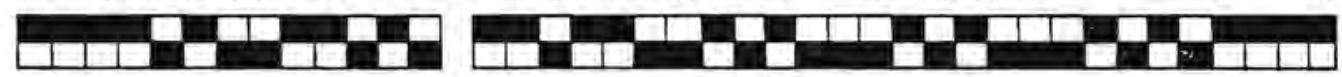

STATISTICAL ACCESS

89

A B A B C D E F G $\quad$ H I

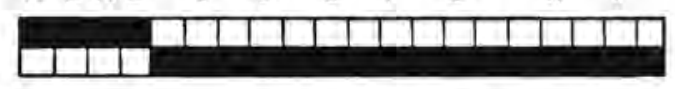

HISTORICAL RECORD
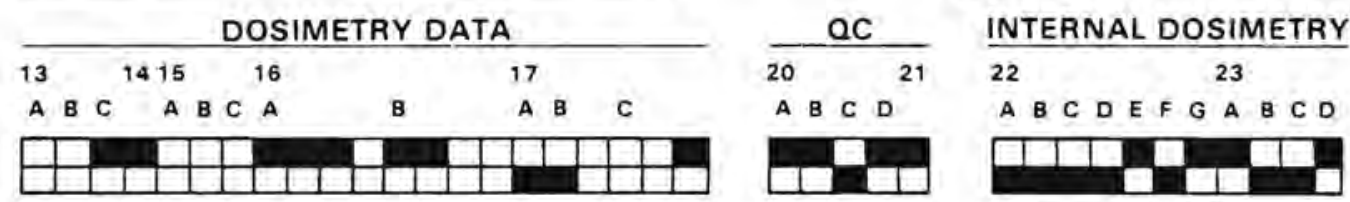

SPECIFIC QUESTIONS AND ANSWERS

\#12. DOE MANUAL 0230

\#17. A. NONPENETRATION DOSE AT - NOT ANSWERED

B. PENETRATING dose AT - NOT ANSWERED

\#18. A. THERMAL NEUTRON QUALITY FACTOR -

B. FAST NEUTRON QUALITY FACTOR -

C. TOTAL NEUTRON QUALITY FACTOR - 6.10

\#19. FILTRATION FILTERS $\# 1-103 \mathrm{mg} \mathrm{cm}^{2}$ PLASTIC

$\# 2.244 \mathrm{mg} \mathrm{cm}{ }^{2}$ TOTAL PLASTIC $.843 \mathrm{mg} \mathrm{cm}^{2} \mathrm{Ta}$

\#3. $1065 \mathrm{mg} \mathrm{cm} \mathrm{cm}^{2}$. PLASTIC $\cdot \mathrm{Cd} \cdot \mathrm{Ta} \cdot$ PAPER

\#4.507 $\mathrm{mg} \mathrm{cm} \mathrm{cm}^{2}$ TOTAL PLASTIC

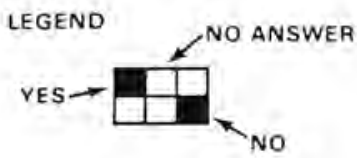


FACILITY - T

TYPE - NATIONAL LABORATORY

DOSIMETRY VOLUME - MEDIUM

DOSIMETRY SERVICE - INHOUSE

\section{SECTION I}

DOSIMETRY DOCUMENTATION

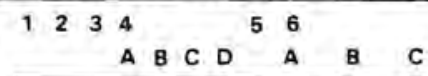

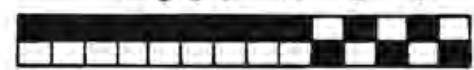

SECTION II

DOSIMETRY PRACTICES

$\begin{array}{lllllll}1 & 2 & 3 & 4 & 5 & 6\end{array}$

A B C A B C D A B A B

ABCDEFGHIJ KLMNOP

RECORD SYSTEM
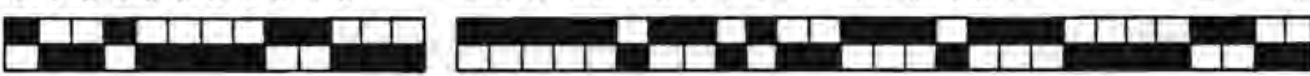

STATISTICAL ACCESS

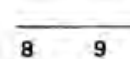

STATISTICALACCESS

HISTORICAL RECORD

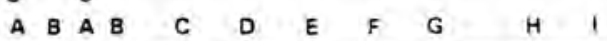

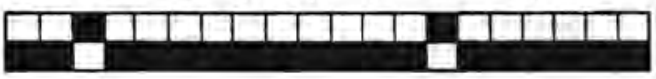

1011
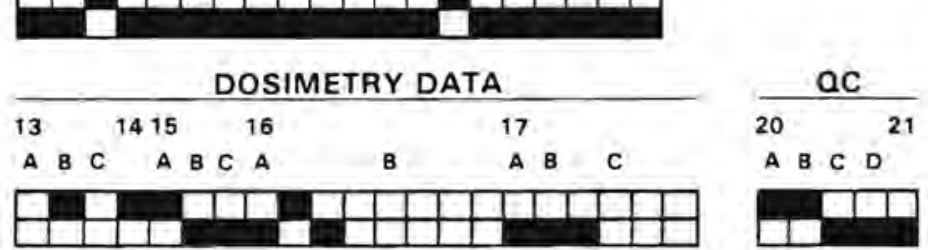

$\frac{\text { INTERNAL DOSIMETRY }}{22}$

A B C DEFG A B C D

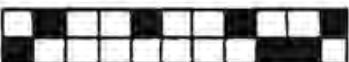

SPECIFIC QUESTIONS AND ANSWERS

\#12. DOE MANUAL CHAPTER 0524

\#17. A. NONPENETRATION DOSE AT $=52 \mathrm{mg} / \mathrm{cm}^{2}$

B. PENETRATING DOSE AT - $\quad 322 \mathrm{mg} / \mathrm{cm}^{2}$

\#18. A. THERMAL NEUTRON QUALITY FACTOR -

B. FAST NEUTRON QUALITY FACTOR -

C. TOTAL NEUTRON QUALITY FACTOR -

\#19. NONPENETRATING FILTER \#1-52mg $/ \mathrm{cm}^{2}$ PLASTIC \#2.

PENETRATING

FILTER \#1 - $52 \mathrm{mg} / \mathrm{cm}^{2}$ PLASTIC

$\# 2 \cdot 270 \mathrm{mg} / \mathrm{cm}^{2} \mathrm{Al}$

$\# 3$.

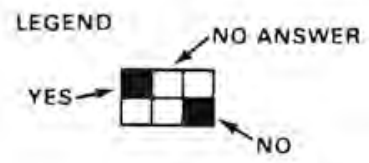

B. 21 
FACILITY - U

TYPE - NATIONAL ENERGY TECHNOLOGY CENTER DOSIMETRY VOLUME - SMALL DOSIMETRY SERVICE - COMMERCIAL

\section{SECTION I}

DOSIMETRY DOCUMENTATION

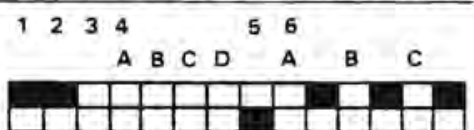

SECTION II

DOSIMETRY PRACTICES

$\begin{array}{llllll}1 & 2 & 3 & 4 & 5 & 6\end{array}$

A B C A B C D A B A B

A B D DEGH I

RECORD SYSTEM

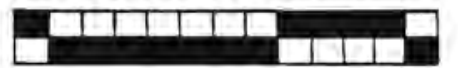

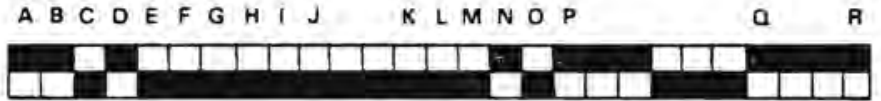

STATISTICAL ACCESS

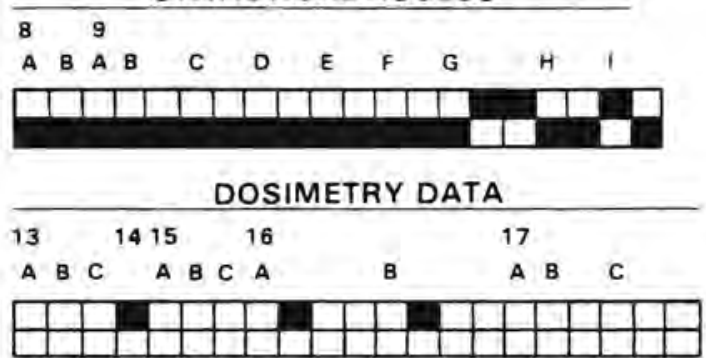

HISTORICAL RECORD

1011

A B A B C D E F G

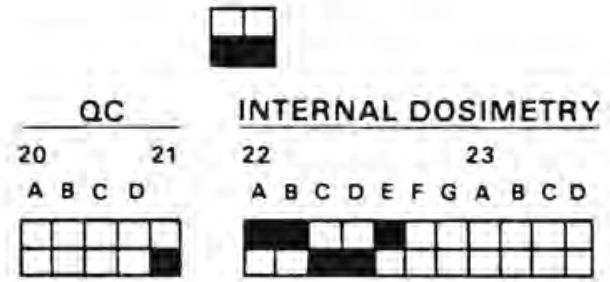

SPECIFIC QUESTIONS AND ANSWERS

\#12. NOT ANSWERED

\#17. A. NONPENETRATION DOSE AT - UNKNOWN

B. PENETRATING DOSE AT - UNKNOWN

\#18. A. THERMAL NEUTRON QUALITY FACTOR -

B. FAST NEUTRON QUALITY FACTOR -

C. TOTAL NEUTRON QUALITY FACTOR.

\#19. NONPENETRATING FILTER \#1.

\#2.

PENETRATING FILTER $\# 1$.

$\# 2$.

$\# 3$.

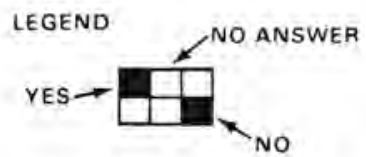


FACILITY - V

TYPE - NATIONAL ENERGY TECHNOLOGY CENTER DOSIMETRY VOLUME - SMALL

DOSIMETRY SERVICE - COMMERCIAL

\section{SECTION I \\ DOSIMETRY DOCUMENTATION

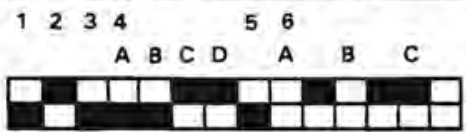

SECTION II

DOSIMETRY PRACTICES

$\begin{array}{lllllll}1 & 2 & 3 & 4 & 5 & 6 & 7\end{array}$

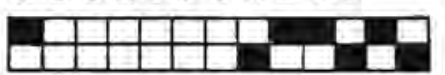

A B C DEFGHII

RECORD SYSTEM

STATISTICAL ACCESS

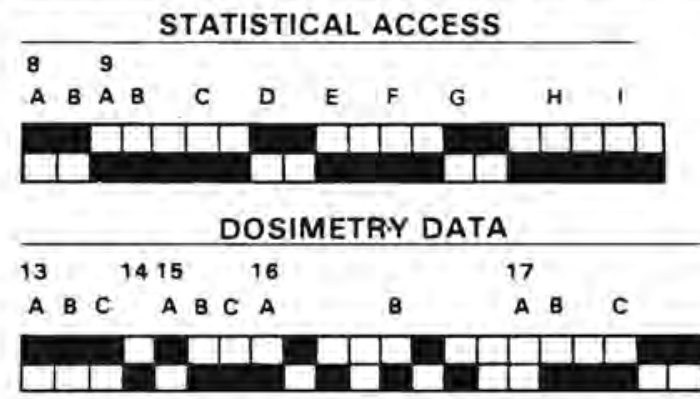

HISTORICAL RECORD

1011

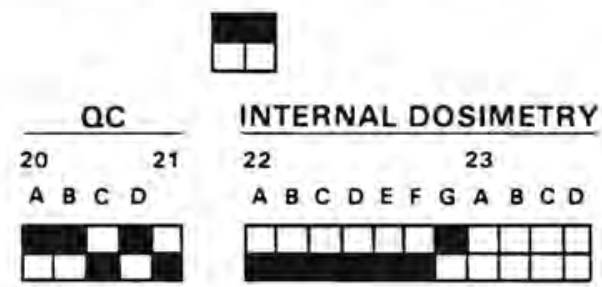

SPECIFIC QUESTIONS AND ANSWERS

\#12. DOE MANUAL CHAPTERS

\#17. A. NONPENETRATION DOSE AT - UNKNOWN

B. PENETRATING DOSE AT - UNKNOWN

\#18. A. THERMAL NEUTRON QUALITY FACTOR -

B. FAST NEUTRON QUALITY FACTOR -

C. TOTAL NEUTRON QUALITY FACTOR .

\#19. NONPENETRATING FILTER \#1.

\#2,

PENETRATING

FILTER $\# 1$.

$\# 2$ -

$\# 3$ -

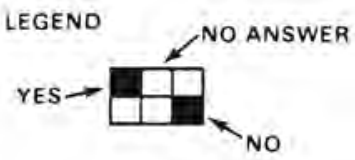


FACILITY - W

TYPE - NATIONAL LABORATORY

DOSIMETRY VOLUME - MEDIUM

DOSIMETRY SERVICE - INHOUSE

\author{
SECTION I \\ DOSIMETRY DOCUMENTATION

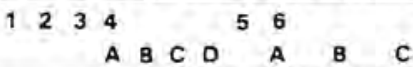

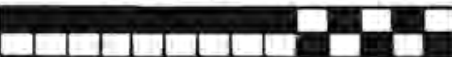

SECTION II

DOSIMETRY PRACTICES

$\begin{array}{llllll}1 & 2 & 3 & 4 & 5 & 6\end{array}$

A B C A B C D A B A B

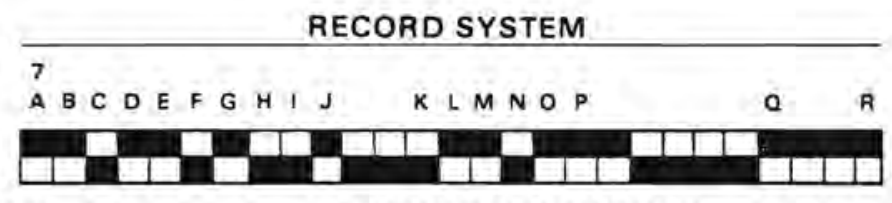

STATISTICAL ACCESS

89

A B A B C C D E F G

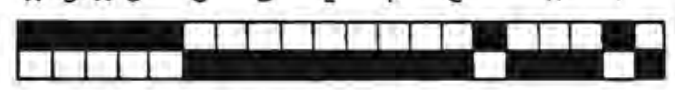

HISTORICAL RECORD
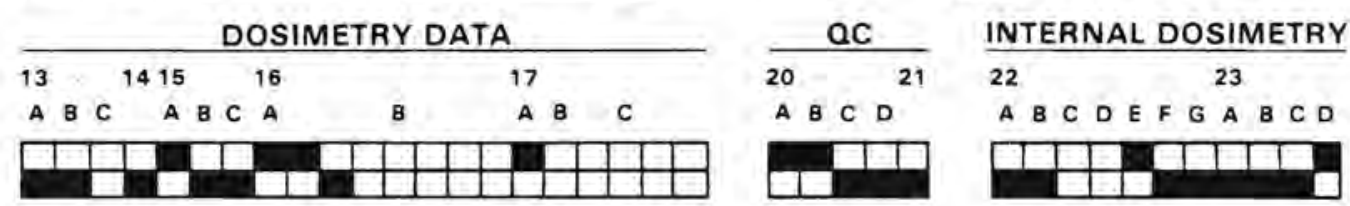

SPECIFIC QUESTIONS AND ANSWERS

\#12. DOE APPENDIX 0230

\#17. A. NONPENETRATION DOSE AT - $7 \mathrm{mg} / \mathrm{cm}^{2}$

B. PENETRATING DOSE AT - UNKNOWN

\#18. A. THERMAL NEUTRON QUALITY FACTOR -

B. FAST NEUTRON QUALITY FACTOR -

C. TOTAL NEUTRON QUALITY FACTOR -

\#19. NONPENETRATING FILTER \#1-55.3mg/ $\mathrm{cm}^{2}$ PLASTIC

$\# 2-23.7 \mathrm{mg} \mathrm{cm}^{2}$ PAPER

PENETRATING FILTER \#1-0.051 cm PLASTIC

$0.038 \mathrm{~cm} \mathrm{Cd}$

$0.013 \mathrm{~cm} \mathrm{Au}$

$0,038 \mathrm{~cm} \mathrm{Cd}$

$0.066 \mathrm{~cm}$ PLASTIC

LEGEND NOANSWER

$0.025 \mathrm{~cm}$ PAPER 
FACILITY - X

TYPE - CONTRACTOR LABORATORY

DOSIMETRY VOLUME - MEDIUM

DOSIMETRY SERVICE - COMMERCIAL

\section{SECTION I}

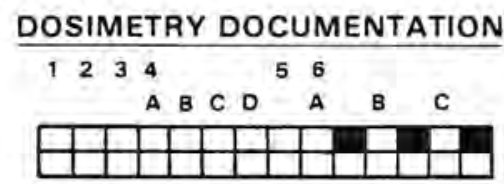

SECTION II

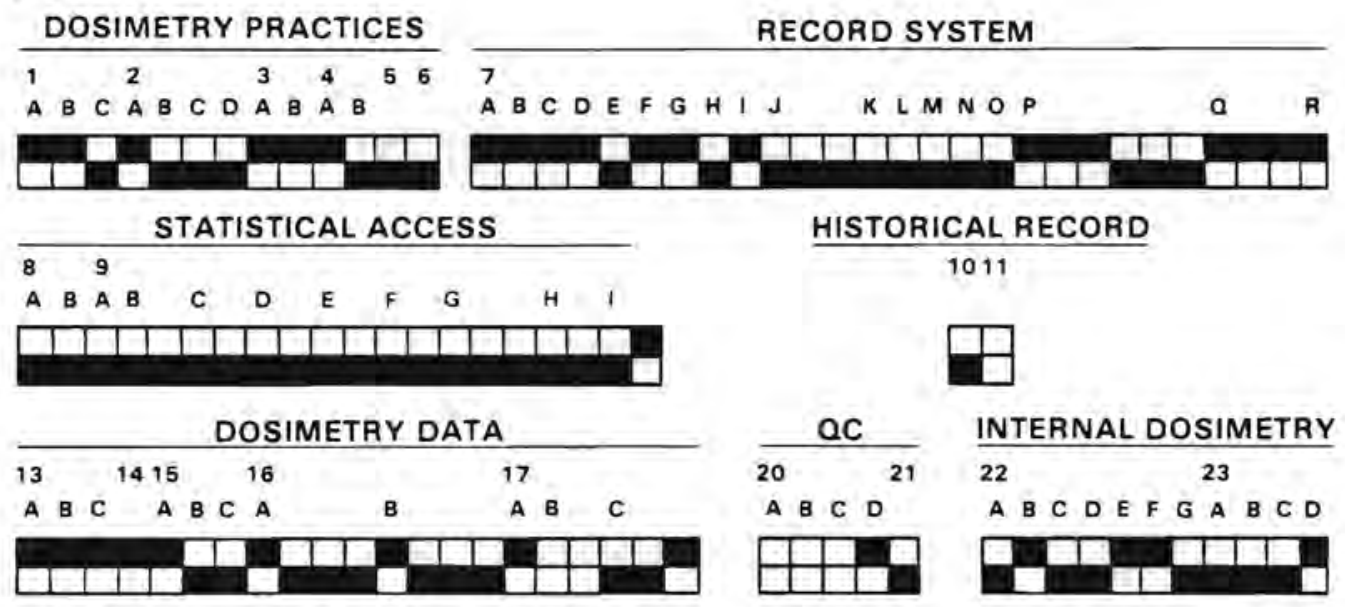

SPECIFIC QUESTIONS AND ANSWERS

\#12. DOE MANUAL CHAPTERS

\#17. A. NONPENETRATION DOSE AT $-7 \mathrm{mg} / \mathrm{cm}^{2}$

B. PENETRATING dose at - UNKNOWN

\#18. A. THERMAL NEUTRON QUALITY FACTOR -

B. FAST NEUTRON QUALITY FACTOR.

C. TOTAL NEUTRON QUALITY FACTOR -

\#19. NONPENETRATING FILTER $\# 1-7 \mathrm{mg} / \mathrm{cm}^{2}$ PLASTIC

\#2.

PENETRATING

FILTER \#1-1 $\mathrm{cm} \mathrm{AI}$

$\# 2$.

$\# 3$.

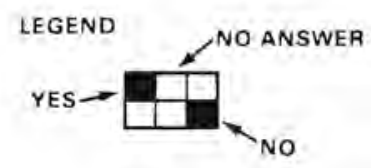




\section{FACILITY - Y}

\section{TYPE - CONTRACTOR}

DOSIMETRY VOLUME - MEDIUM

DOSIMETRY SERVICE - COMMERCIAL

\section{SECTION I}

DOSIMETRY DOCUMENTATION

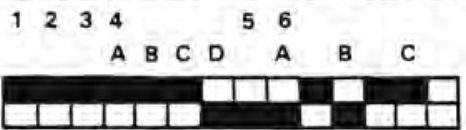

SECTION II

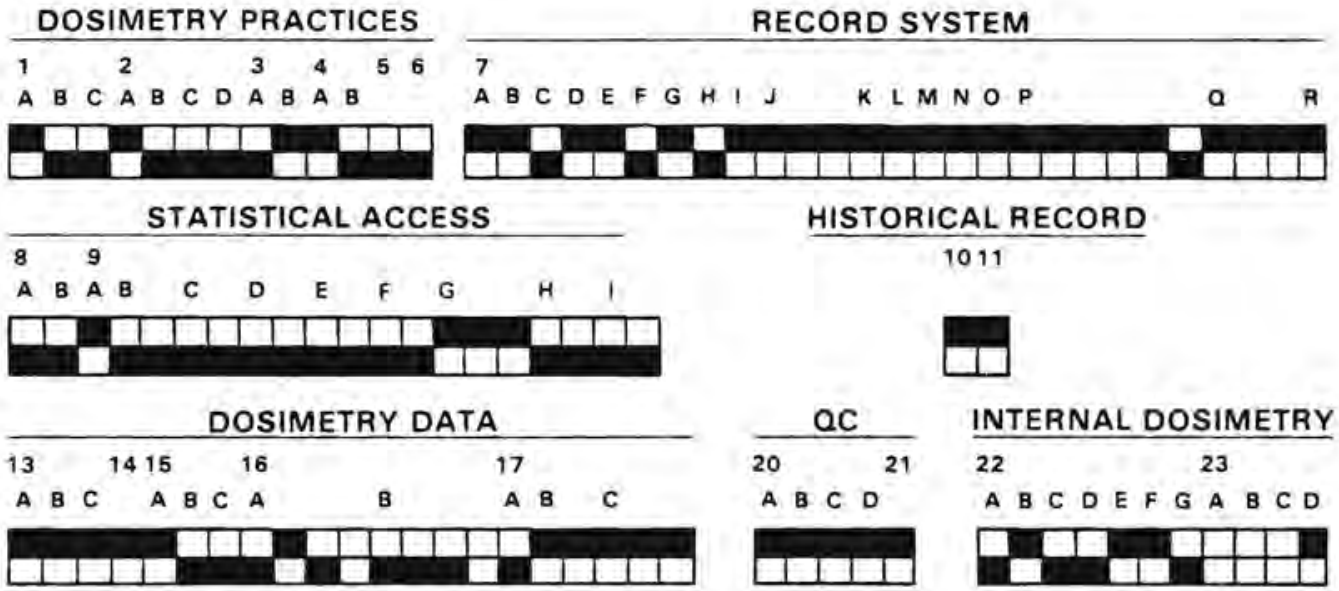

\section{SPECIFIC QUESTIONS AND ANSWERS}

\#12. CORPORATE POLICY - PERMANENT

\#17. A. NONPENETRATION DOSE AT -

B. PENETRATING DOSE AT - $1 \mathrm{~cm}$

\#18. A. THERMAL NEUTRON QUALITY FACTOR - 3
B. FAST NEUTRON QUALITY FACTOR-
C. TOTAL NEUTRON QUALITY FACTOR -

\#19. FILTRATION FILTERS: $100 \mathrm{mg} / \mathrm{cm}^{2}$ PLASTIC

$175 \mathrm{mg} / \mathrm{cm}^{2}$ PLASTIC

$325 \mathrm{mg} / \mathrm{cm}^{2}$ PLASTIC

$375 \mathrm{mg} / \mathrm{cm}^{2} \mathrm{Al}$

$660 \mathrm{mg} / \mathrm{cm}^{2} \mathrm{~Pb}+\mathrm{Sn}$

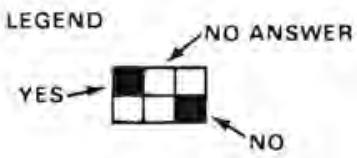




\section{FACILITY - Z}

TYPE - UNIVERSITY

DOSIMETRY VOLUME - MEDIUM

DOSIMETRY SERVICE - COMMERCIAL

\section{SECTION I}

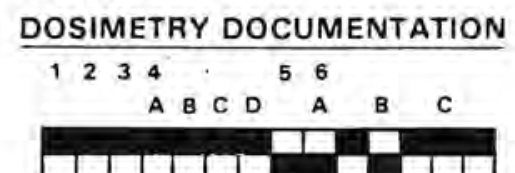

\section{SECTION II}

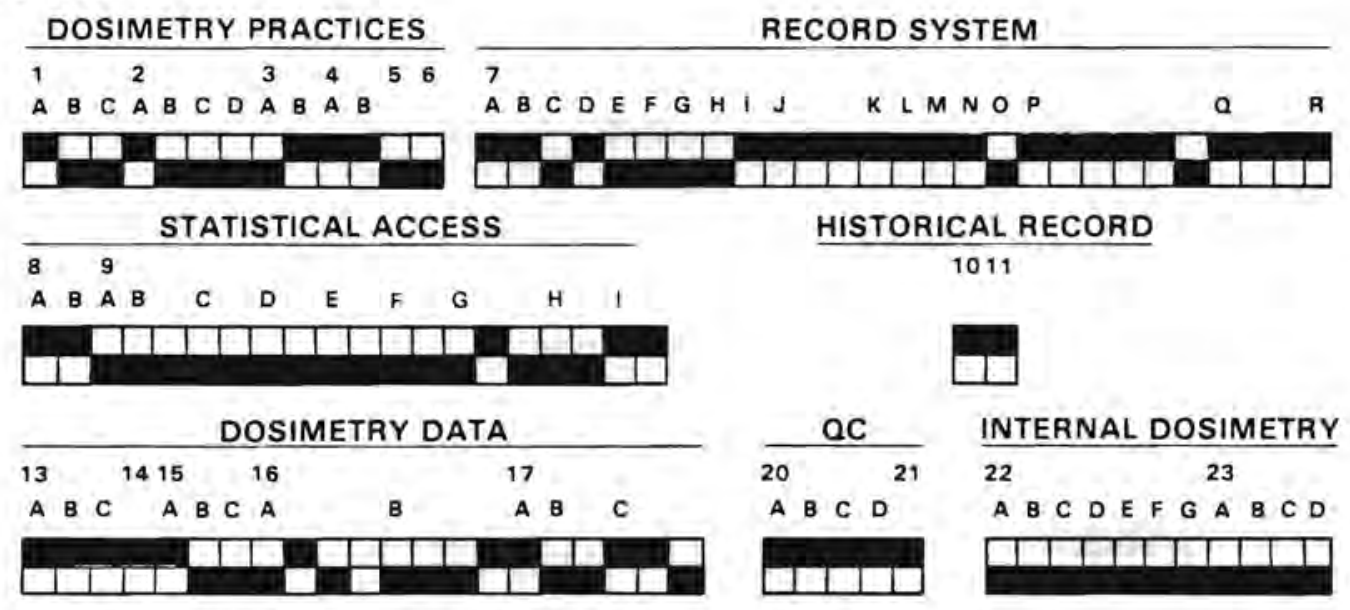

\section{SPECIFIC QUESTIONS AND ANSWERS}

\#12. CODE OF FEDERAL REGULATIONS

\#17. A. NONPENETRATION DOSE AT $-7 \mathrm{mg} / \mathrm{cm}^{2}$

B. PENETRATING DOSE AT -

\#18. A. THERMAL NEUTRON QUALITY FACTOR - $\mathbf{3 . 5}-\mathbf{5}$
B. FAST NEUTRON QUALITY FACTOR-

C. TOTAL NEUTRON QUALITY FACTOR -

\#19. NONPENETRATING FILTER \#1.

$\# 2$.

PENETRATING

FILTER \# 1 .

$\# 2$.

\#3.

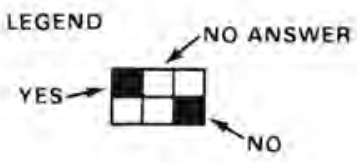


FACILITY - AA

TYPE - UNIVERSITY

DOSIMETRY VOLUME - SMALL

DOSIMETRY SERVICE - COMMERCIAL

\author{
SECTION I \\ DOSIMETRY DOCUMENTATION \\ \begin{tabular}{lllllll}
12 & 3.4 & 5 & 6 \\
\hline & A B C D & A & B & C \\
\hline
\end{tabular}
}

SECTION II

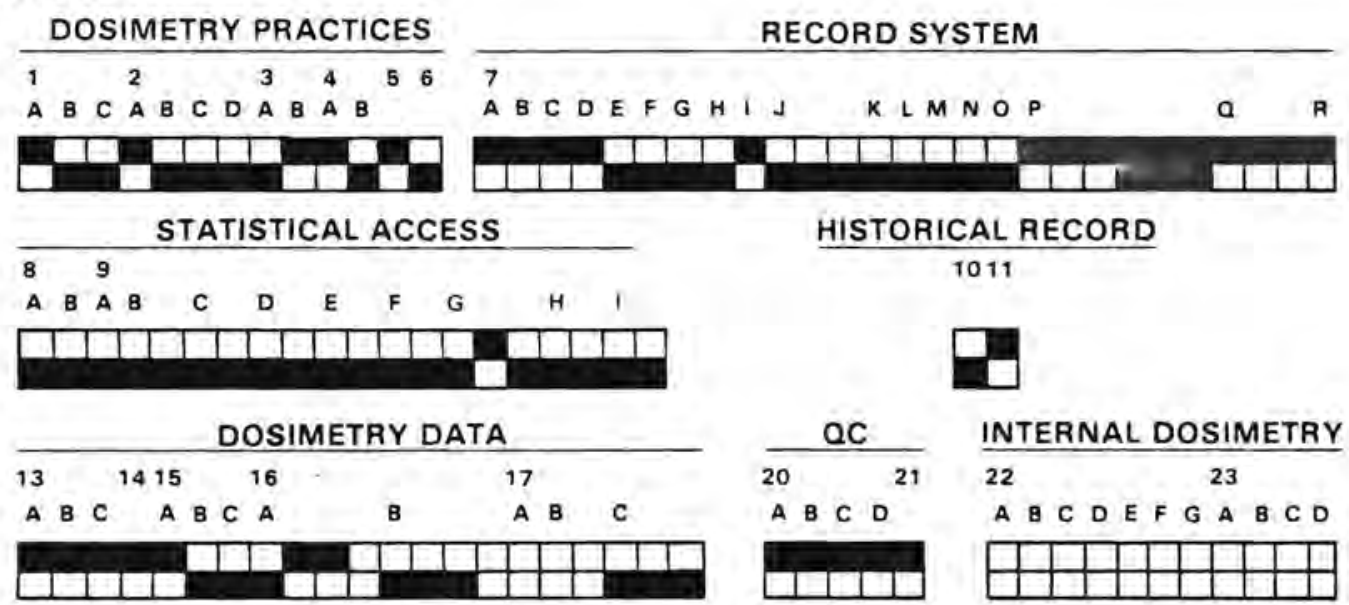

SPECIFIC QUESTIONS AND ANSWERS

\#12. CODE OF FEDERAL REGULATIONS

\#17. A. NONPENETRATION DOSE AT $-25 \mathrm{mg} / \mathrm{cm}^{2}$

B. PENETRATING DOSE AT - UNKNOWN

\#18. A. THERMAL NEUTRON QUALITY FACTOR - 3

B. FAST NEUTRON QUALITY FACTOR - 10

C. TOTAL NEUTRON QUALITY FACTOR -

\#19. NONPENETRATING FILTER \#1-25mg/ $\mathrm{cm}^{2}$ PLASTIC

$\# 2-100 \mathrm{mg} / \mathrm{cm}^{2}$ PLASTIC

$\# 3-175 \mathrm{mg} / \mathrm{cm}^{2}$ PLASTIC

$\# 4-325 \mathrm{mg} / \mathrm{cm}^{2}$ PLASTIC

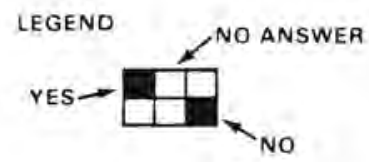


FACILITY - BB

TYPE - UNIVERSITY

DOSIMETRY VOLUME - MEDIUM

DOSIMETRY SERVICE - COMMERCIAL

SECTION I

DOSIMETRY DOCUMENTATION

123

A B C D A B C

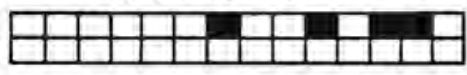

SECTION II

DOSIMETRY PRACTICES

$\begin{array}{llllll}1 & 2 & 3 & 4 & 5 & 6\end{array}$

A B C A B C D A B A B

A B C DEF G H I

RECORD SYSTEM
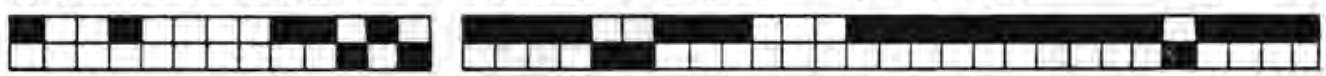

STATISTICAL ACCESS

89

A B A B C D D E F G

HISTORICAL RECORD

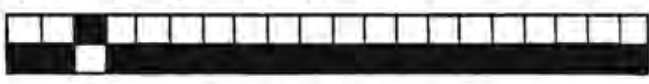

1011
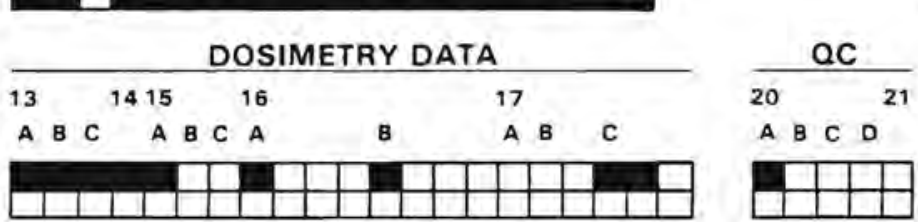

$\square$

$\frac{\text { INTERNAL DOSIMETRY }}{22}$

A B C DEFG A B C D

SPECIFIC QUESTIONS AND ANSWERS

\#12. CODE OF FEDERAL REGULATIONS

\#17. A. NONPENETRATION DOSE AT - NOT ANSWERED - COMMERCIAL SERVICE

B. penetrating dose at - not answered - commercial service

\#18. A. THERMAL NEUTRON QUALITY FACTOR

B. FAST NEUTRON QUALITY FACTOR -

C. TOTAL NEUTRON OUALITY FACTOR -

\#19. NONPENETRATING FILTER $\# 1$,

$\# 2$.

PENETRATING FILTER \#1-

$\# 2$.

$\# 3$

LEGEND NO ANSWER 
FACILITY - CC

TYPE - CONTRACTOR LABORATORY

DOSIMETRY VOLUME - SMALL

DOSIMETRY SERVICE - COMMERCIAL

\section{SECTION I \\ DOSIMETRY DOCUMENTATION \\ 123 \\ A B C D A B C

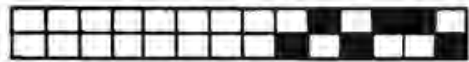

\section{SECTION II}

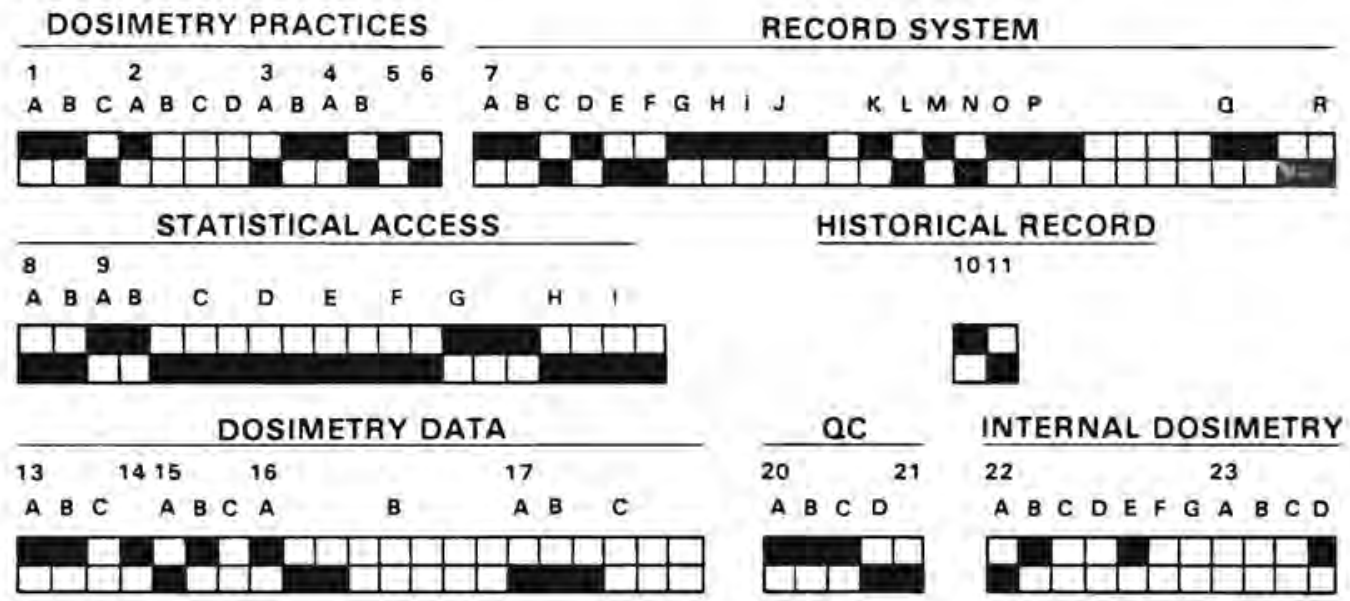

SPECIFIC QUESTIONS AND ANSWERS

\#12. DOE MANAUL CHAPTER 0525

\#17. A. NONPENETRATION DOSE AT - $10 \mathrm{mg} / \mathrm{cm}^{2}$

B. PENETRATING DOSE AT, $\quad 285 \mathrm{mg} / \mathrm{cm}^{2}$

\#18. A. THERMAL NEUTRON QUALITY FACTOR - NOT APPLICABLE

B. FAST NEUTRON QUALITY FACTOR -

C. TOTAL NEUTRON QUALITY FACTOR -

\#19. NONPENETRATING FILTER \#1-10 $\mathrm{mg} / \mathrm{cm}^{2}$ MYLAR AND PAPER

\#2,

PENETRATING

FILTER \#1- $285 \mathrm{mg} / \mathrm{cm}^{2}$ ALUMINUM

$\# 2$ -

\#3-

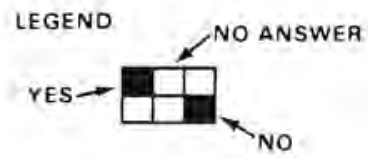


FACILITY - DD

TYPE - UNIVERSITY

DOSIMETRY VOLUME - MEDIUM

DOSIMETRY SERVICE - COMMERCIAL

\section{SECTION I}

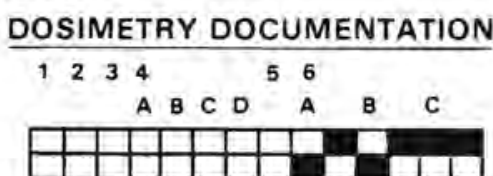

SECTION II

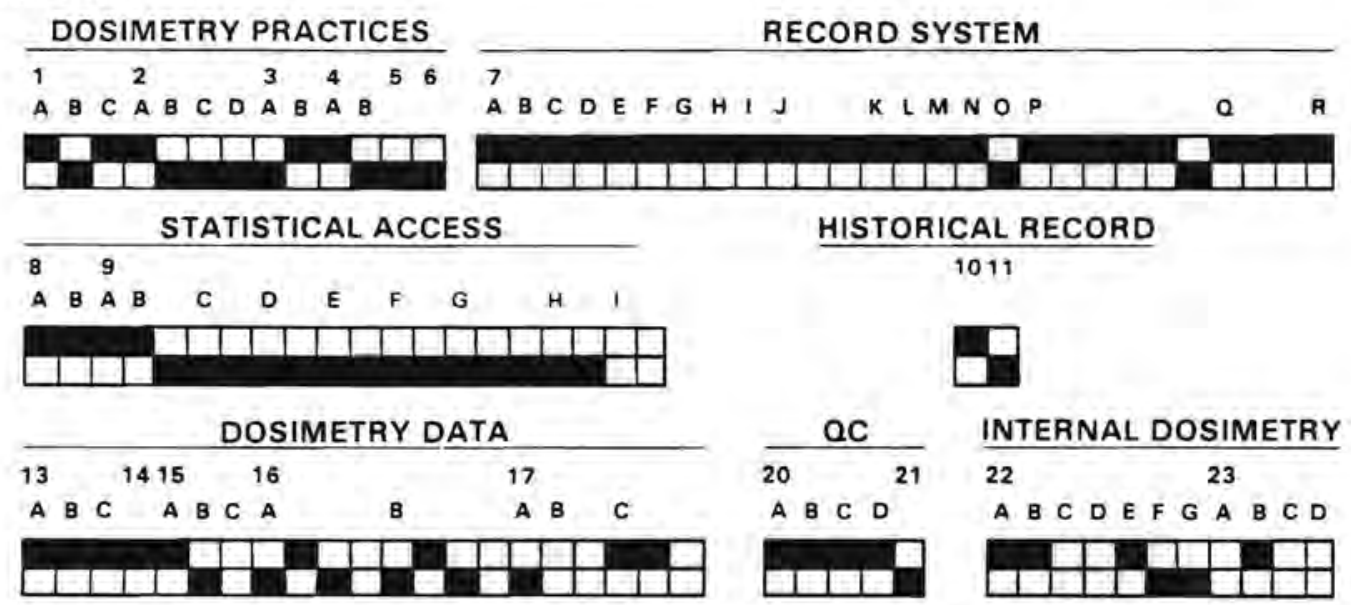

\section{SPECIFIC QUESTIONS AND ANSWERS}

\#12. DOE MANUAL CHAPTER 0525

\#17. A. NONPENETRATION DOSE AT $-25 \mathrm{mg} / \mathrm{cm}^{2}$

B. PENETRATING DOSE AT - ADJUSTED TO GAMMA ENERGY

\#18. A. THERMAL NEUTRON QUALITY FACTOR - 3

B. FAST NEUTRON QUALITY FACTOR -

C. TOTAL NEUTRON QUALITY FACTOR -

\#19. NONPENETRATING FILTER \#1-25mg $/ \mathrm{cm}^{2}$ PLASTIC

AND

PENETRATING

$\# 2-100 \mathrm{mg} / \mathrm{cm}^{2}$ PLASTIC

$\# 3-175 \mathrm{mg} / \mathrm{cm}^{2}$ PLASTIC

\#4 - $325 \mathrm{mg} / \mathrm{cm}^{2}$ PLASTIC

\#5 - $300 \mathrm{mg} / \mathrm{cm}^{2} \mathrm{Al}+75 \mathrm{mg} / \mathrm{cm}^{2}$ PLASTIC

\#6 - $1520 \mathrm{mg} / \mathrm{cm}^{2} \mathrm{~Pb}(60 \%)$ AND $\mathrm{Sn}(40 \%)+140 \mathrm{mg} / \mathrm{cm}^{2}$ PLASTIC

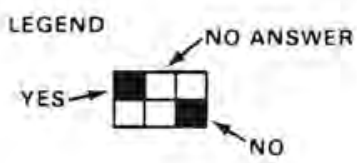


FACILITY - EE

TYPE - UNIVERSITY

DOSIMETRY VOLUME - MEDIUM

DOSIMETRY SERVICE - COMMERCIAL

\section{SECTION I \\ DOSIMETRY DOCUMENTATION

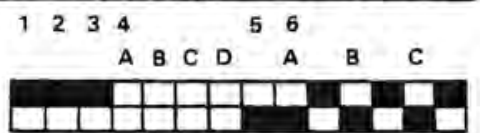

SECTION II

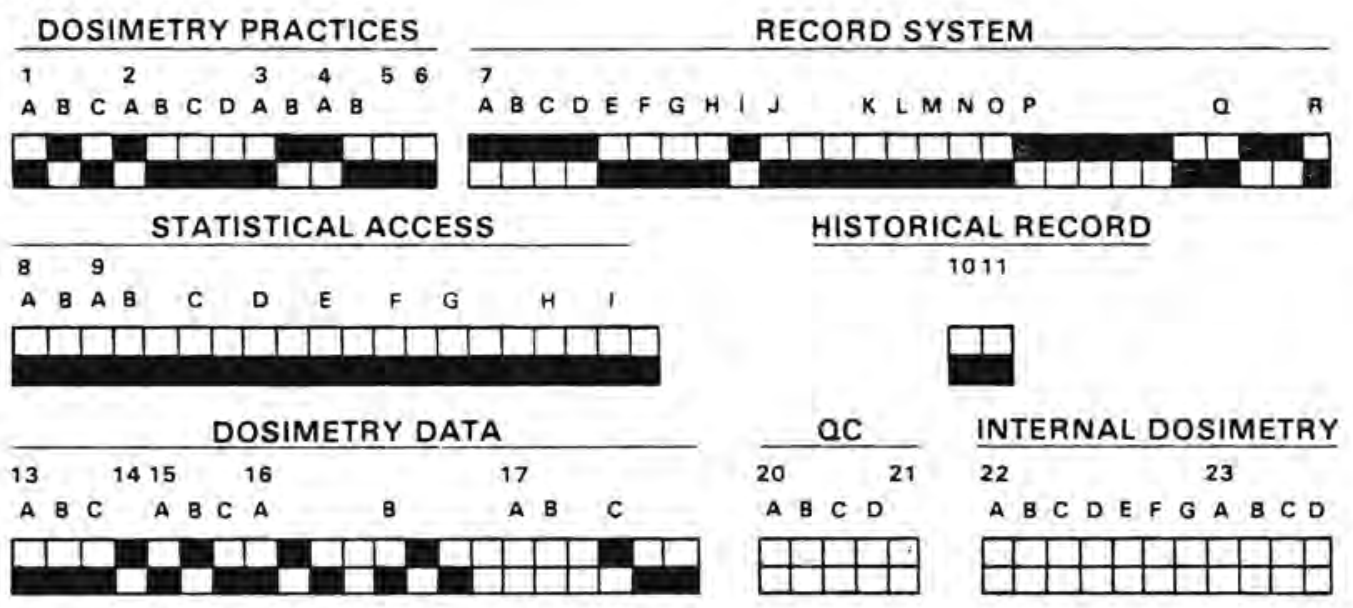

SPECIFIC QUESTIONS AND ANSWERS

\#12. CODE OF FEDERAL REGULATIONS

\#17. A. NONPENETRATION DOSE AT - UNKNOWN

B. PENETRATING DOSE AT - UNKNOWN

\#18. A. THERMAL NEUTRON QUALITY FACTOR - NOT ANSWERED

B. FAST NEUTRON QUALITY FACTOR - NOT ANSWERED

C. TOTAL NEUTRON QUALITY FACTOR - NOT ANSWERED

\#19. NONPENETRATING FILTER \#1 - NOT ANSWERED

$\# 2$.

PENETRATING FILTER \#1.

$\# 2$ -

\#3.

LEGEND 
FACILITY - FF

TYPE - UNIVERSITY

DOSIMETRY VOLUME - SMALL

DOSIMETRY SERVICE - COMMERCIAL

\section{SECTION I}

$\frac{\text { DOSIMETRY DOCUMENTATION }}{1234}$

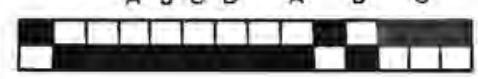

SECTION II

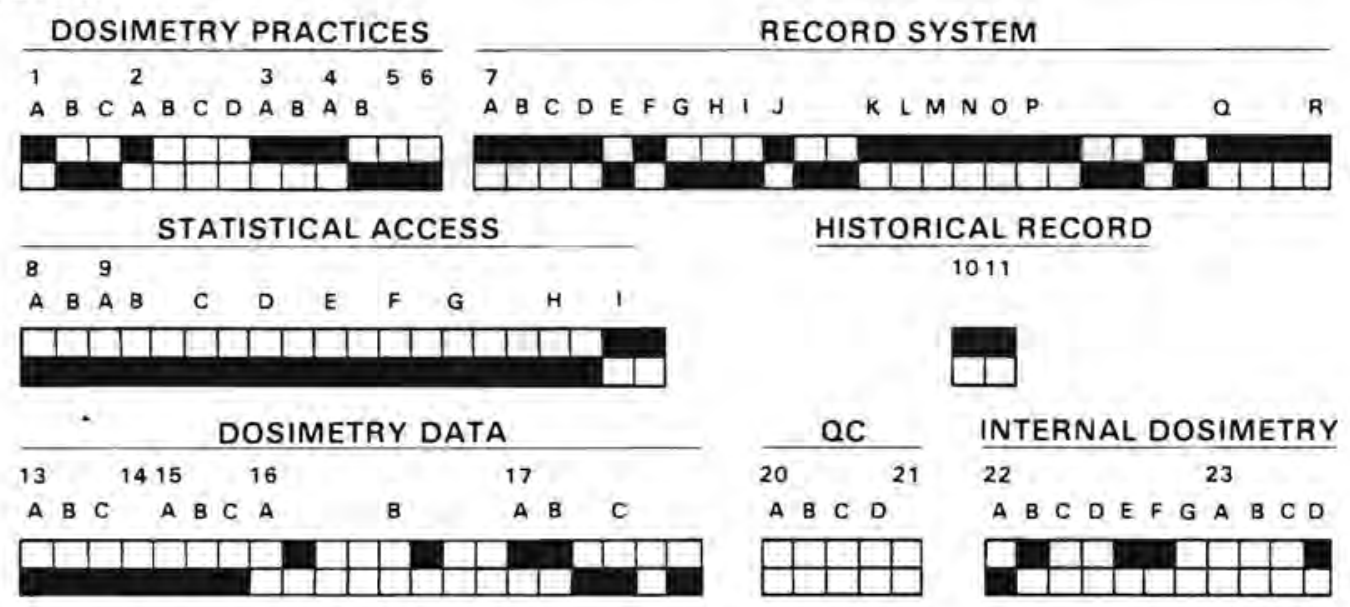

\section{SPECIFIC QUESTIONS AND ANSWERS}

\#12. CODE OF FEDERAL REGULATIONS (ALL RECORDS KEPT PERMANENTLY)

\#17. A. NONPENETRATION DOSE AT $-7 \mathrm{mg} / \mathrm{cm}^{2}$

B. Penetrating dose at - $1 \mathrm{~cm}$

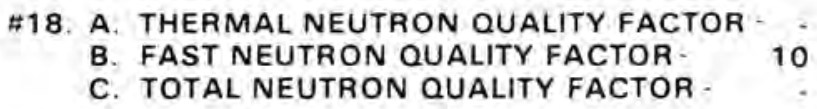

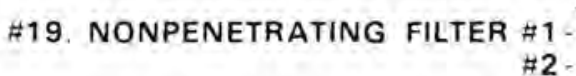

PENETRATING

FILTER $\left.\begin{array}{rl} & \# 1- \\ & \# 2- \\ & \# 3-\end{array}\right\}$ REFER TO COMMERCIAL VENDER

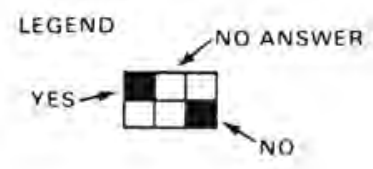


FACILITY - GG

TYPE - UNIVERSITY

DOSIMETRY VOLUME - SMALL

DOSIMETRY SERVICE - COMMERCIAL

\section{SECTION I \\ $\frac{\text { DOSIMETRY DOCUMENTATION }}{1233^{4}}$ \\ $A B C D$ A $B$ C

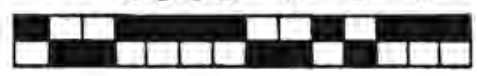

SECTION II

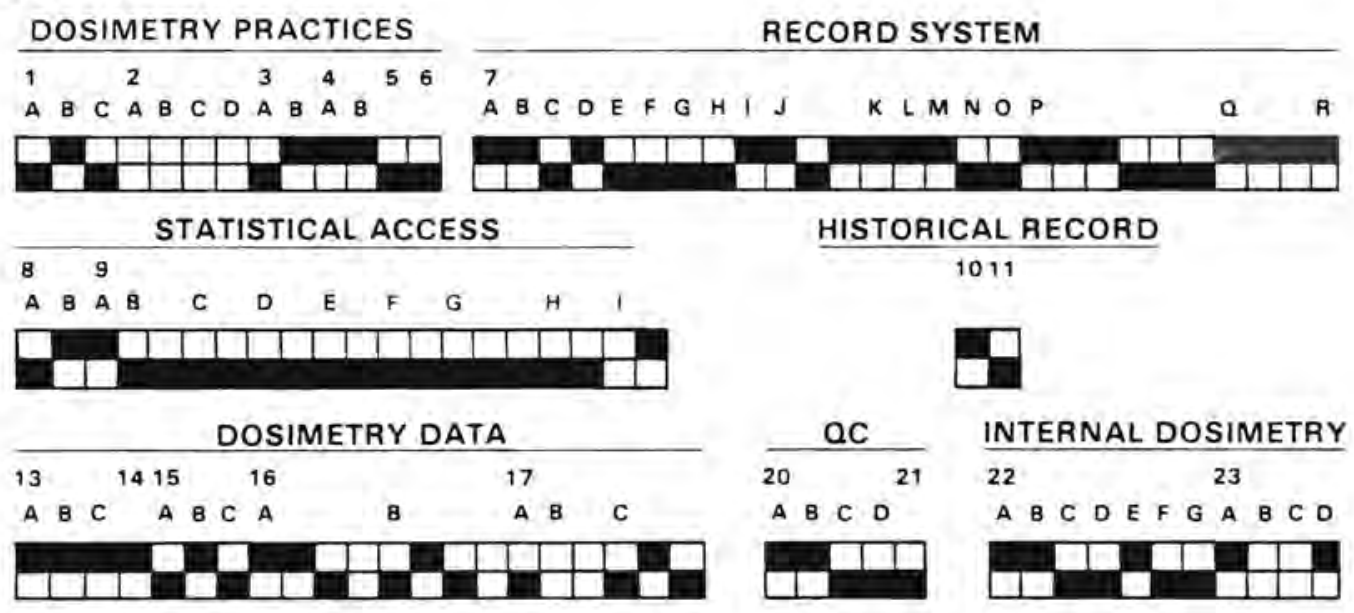

\section{SPECIFIC QUESTIONS AND ANSWERS}

\#12. UNIVERSITY POLICY

\#17. A. NONPENETRATION DOSE AT - UNKNOWN

B. PENETRATING DOSE AT - UNKNOWN

\#18. A. THERMAL NEUTRON QUALITY FACTOR-

B. FAST NEUTRON QUALITY FACTOR-

C. TOTAL NEUTRON QUALITY FACTOR - NORMALIZE TO $4 \mathrm{MeV}$

\#19. NONPENETRATING FILTER \#1- PAPER WRAPPER

$\# 2$.

\begin{tabular}{|c|c|}
\hline PENETRATING & 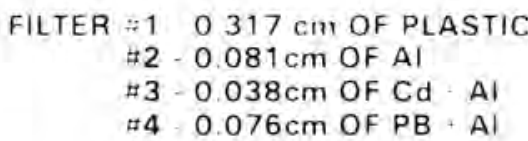 \\
\hline
\end{tabular}

LEGEND 
FACILITY - HH

TYPE - CONTRACTOR LABORATORY

DOSIMETRY VOLUME - SMALL

DOSIMETRY SERVICE - COMMERCIAL

\section{SECTION I \\ DOSIMETRY DOCUMENTATION \\ 123 \\ A B C D A B C

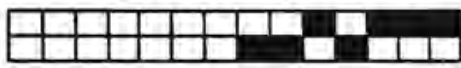

SECTION II

DOSIMETRY PRACTICES

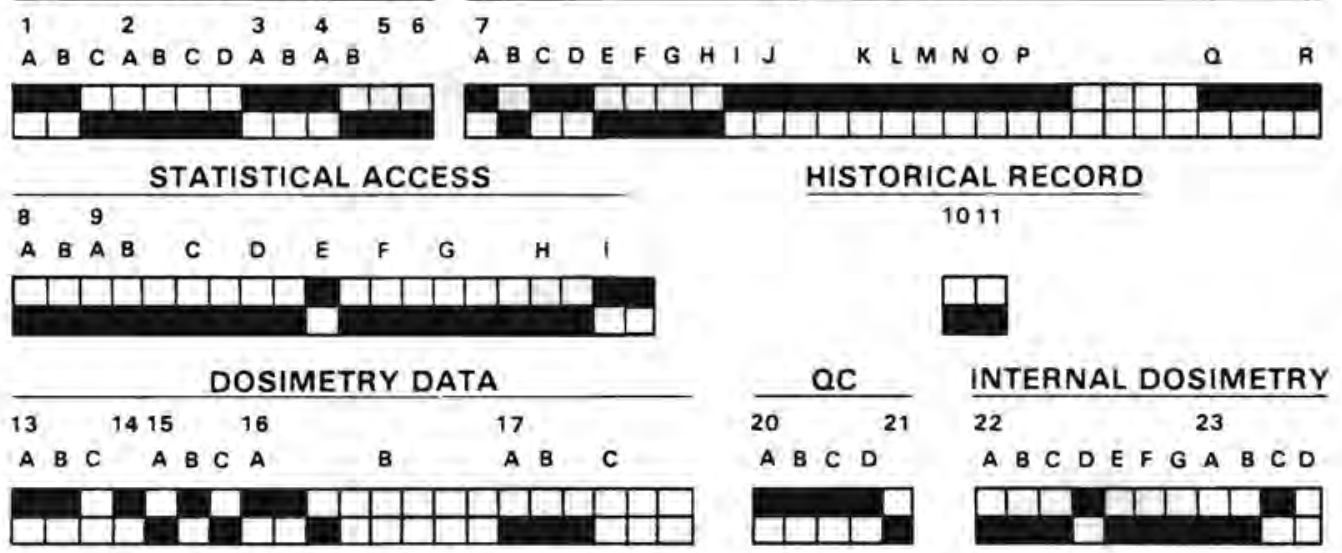

SPECIFIC QUESTIONS AND ANSWERS

\#12. CONTRACTOR POLICY

\#17. A. NONPENETRATION DOSE AT.

B. PENETRATING DOSE AT .

\#18. A. THERMAL NEUTRON QUALITY FACTOR-

B. FAST NEUTRON QUALITY FACTOR -

C. TOTAL NEUTRON QUALITY FACTOR -

\#19. NONPENETRATING FILTER \#1- $20 \mathrm{mg} / \mathrm{cm}^{2}$ PLASTIC

$\# 2$.

PENETRATING FILTER \#1- $0.9 \mathrm{~cm}$ OF Pb

$\# 2$ - $1 \mathrm{~cm}$ OF $\mathrm{Al}$

\#3-

LEGENO NO ANSWER 
FACILITY - II

TYPE - CONTRACTOR LABORATORY

DOSIMETRY VOLUME - SMALL

DOSIMETRY SERVICE - UNIVERSITY

\section{SECTION I \\ DOSIMETRY DOCUMENTATION \\ 12345 \\ A B C D A B C

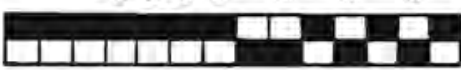

SECTION II

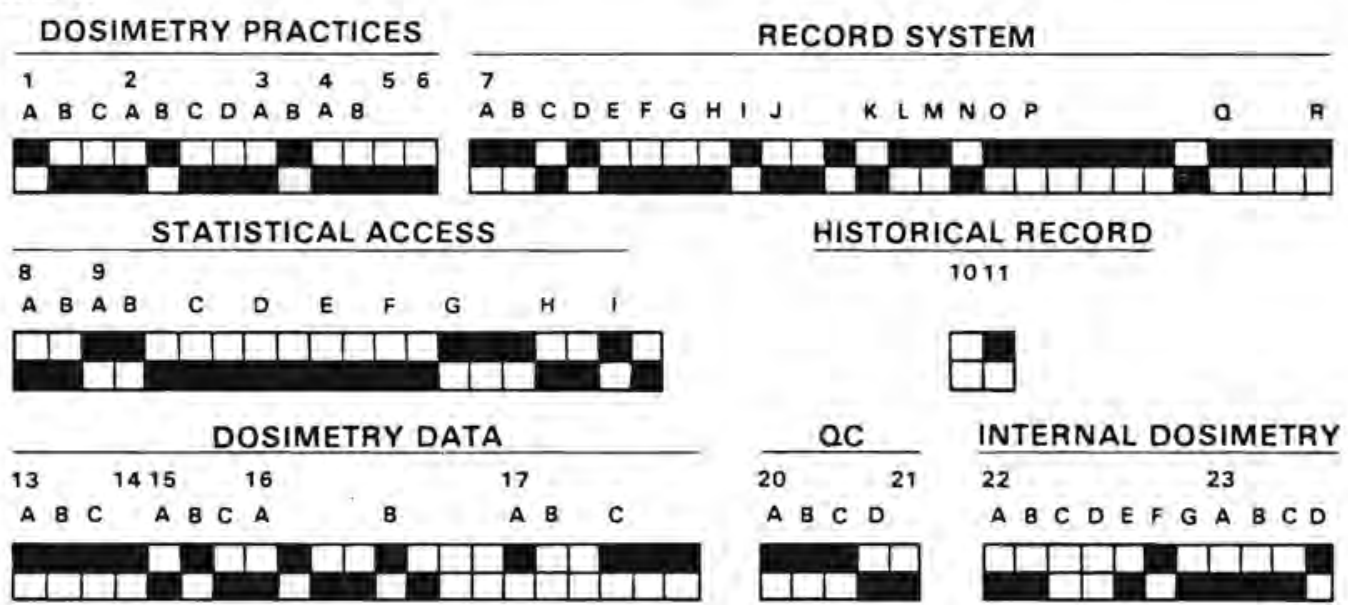

SPECIFIC QUESTIONS AND ANSWERS

\#12. CODE OF FEDERAL REGULATIONS

\#17. A. NONPENETRATION DOSE AT $-7 \mathrm{mg} / \mathrm{cm}^{2}$

B. PENETRATING DOSE AT - UNKNOWN

\#18. A. THERMAL NEUTRON QUALITY FACTOR - 7.5 FOR CF-252

B. FAST NEUTRON QUALITY FACTOR-

C. TOTAL NEUTRON QUALITY FACTOR -

\#19. NONPENETRATING FILTER \#1-0

\#2.

PENETRATING FILTER $\# 1-0.1 \mathrm{~cm} \mathrm{Cd}$

$\# 2$.

\#3 -<smiles>[R15]OC(=O)O[Na]</smiles> 
FACILITY - JJ

TYPE - UNIVERSITY

DOSIMETRY VOLUME - SMALL

DOSIMETRY SERVICE - COMMERCIAL

SECTION I

DOSIMETRY DOCUMENTATION

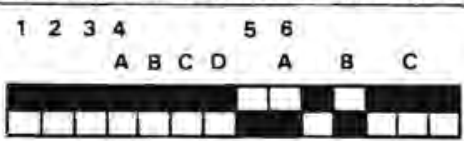

SECTION II

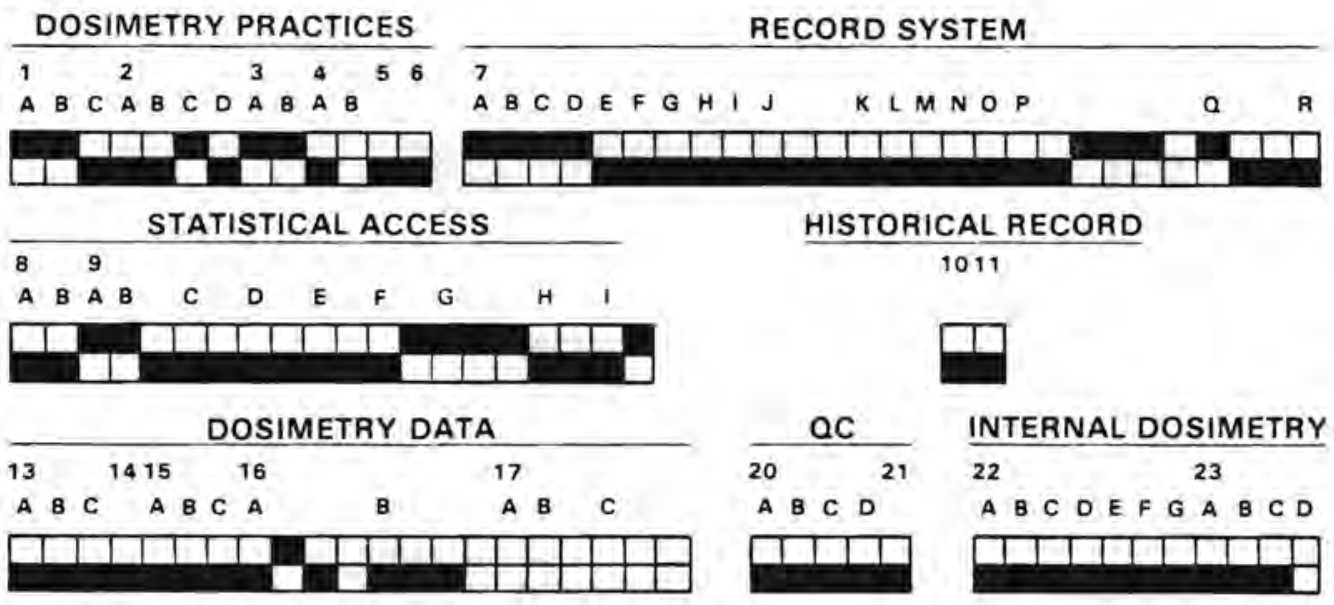

SPECIFIC QUESTIONS AND ANSWERS

$\$ 12$.

417. A. NONPENETRATION DOSE AT - UNKNOWN

B. PENETRATING DOSE AT - UNKNOWN

\#18. A. THERMAL NEUTRON QUALITY FACTOR

B. FAST NEUTRON QUALITY FACTOR.

C. TOTAL NEUTRON QUALITY FACTOR -

\%19. NONPENETRATING FILTER \#1

$\# 2$.

PENETRATING FILTER $\# 1$.

\#2.

$\# 3$.

LEGEND 
FACILITY - KK

TYPE - UNIVERSITY

DOSIMETRY VOLUME - SMALL

DOSIMETRY SERVICE - COMMERCIAL

\section{SECTION I \\ DOSIMETRY DOCUMENTATION \\ $A B C D$ A B C

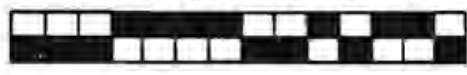

SECTION II

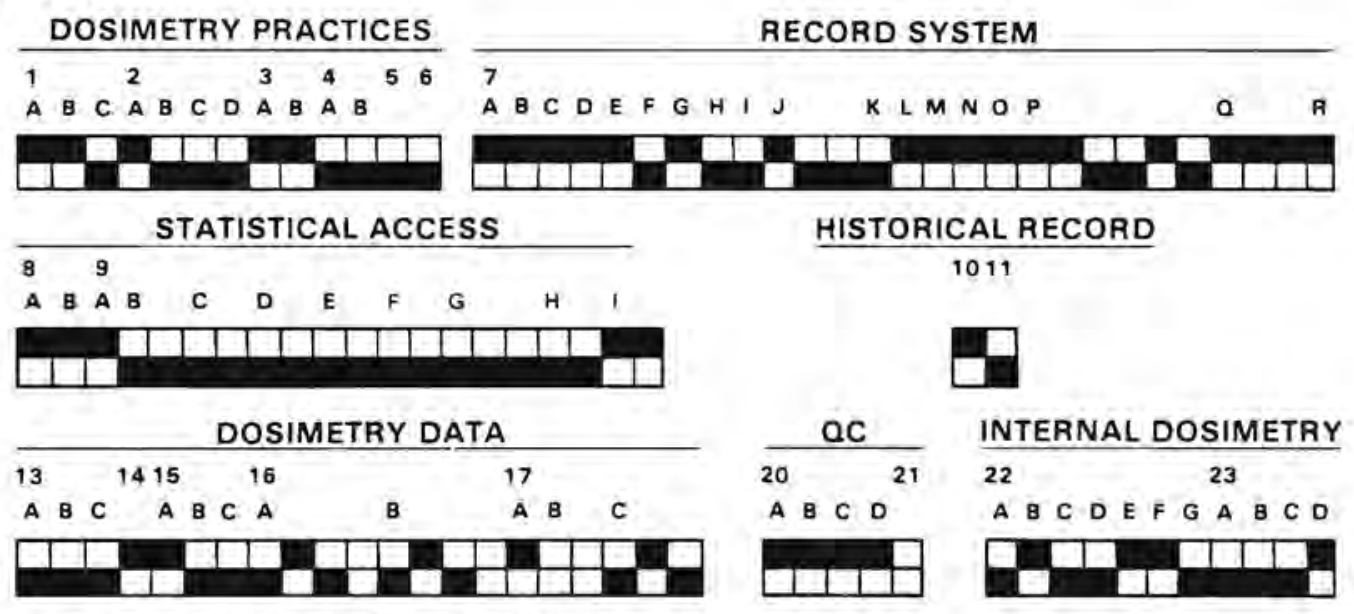

SPECIFIC QUESTIONS AND ANSWERS

\#12. DOE MANAUL CHAPTERS

\#17. A. NONPENETRATION DOSE AT $-7 \mathrm{mg} / \mathrm{cm}^{2}$

B. PENETRATING DOSE AT - UNKNOWN

\#18. A. THERMAL NEUTRON QUALITY FACTOR -

B. FAST NEUTRON QUALITY FACTOR - 10

C. TOTAL NEUTRON QUALITY FACTOR -

\#19. NONPENETRATING FILTER \#1.

$$
\# 2 \text {. }
$$

PENETRATING FILTER \#1

$\# 2$.

$\#$ 3-

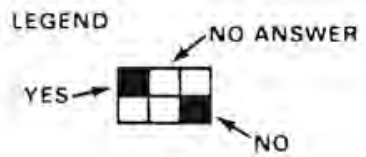


FACILITY - LL

TYPE - UNIVERSITY

DOSIMETRY VOLUME - SMALL

DOSIMETRY SERVICE - UNIVERSITY

\author{
SECTION I \\ DOSIMETRY DOCUMENTATION

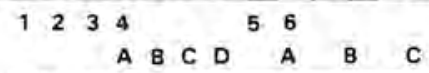

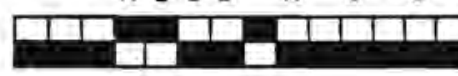

SECTION II

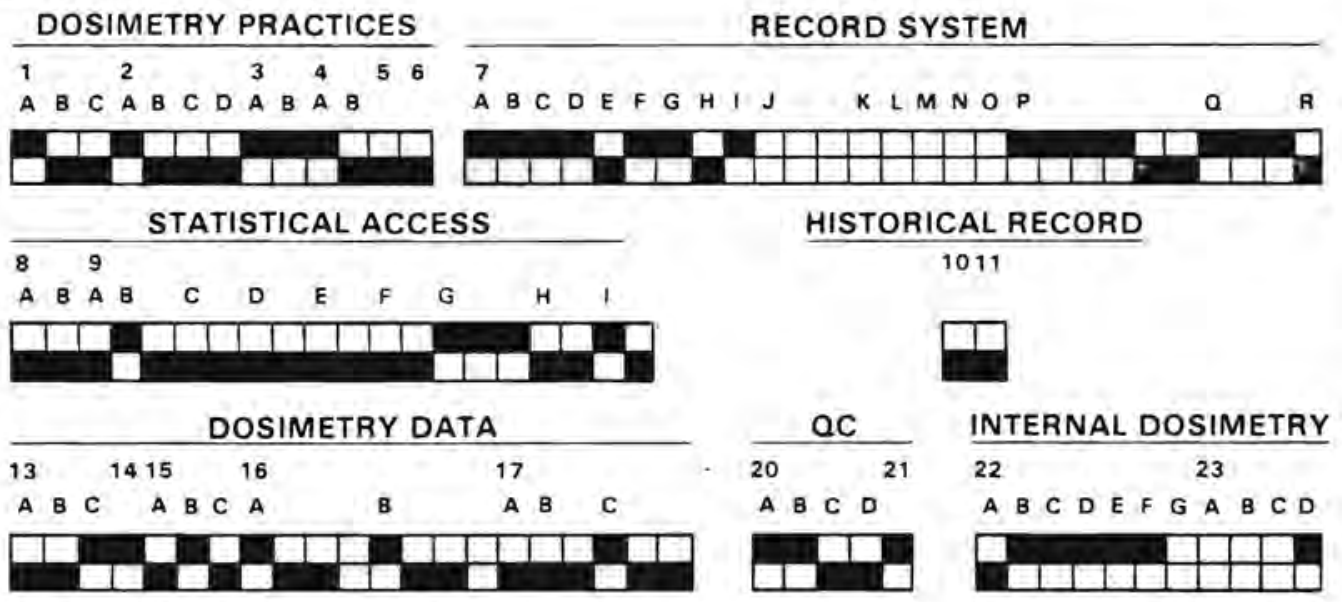

\title{
SPECIFIC QUESTIONS AND ANSWERS
}

\#12. CODE OF FEDERAL REGULATIONS

\#17. A. NONPENETRATION DOSE AT $\cdot 10 \mathrm{mg} / \mathrm{cm}^{2}$

B. PENETRATING DOSE AT - $285 \mathrm{mg} / \mathrm{cm}^{2}$

\#18. A. THERMAL NEUTRON QUALITY FACTOR - 10

B. FAST NEUTRON QUALITY FACTOR .

C. TOTAL NEUTRON QUALITY FACTOR -

\#19. NONPENETRATING FILTER \#1-10mg/ $\mathrm{cm}^{2}$ MYLAR

\#2.

PENETRATING FILTER \#1-285mg $/ \mathrm{cm}^{2}$ AI

$\# 2$.

$\# 3$.

LEGEND NO ANSWER 
FACILITY - MM

TYPE - CONTRACTOR LABORATORY

DOSIMETRY VOLUME - MEDIUM

DOSIMETRY SERVICE - INHOUSE

\section{SECTION I \\ $\frac{\text { DOSIMETRY DOCUMENTATION }}{1234}$ \\ A B C D $A$ B $\quad$ C

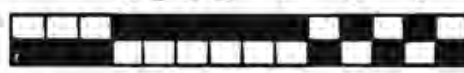

SECTION II

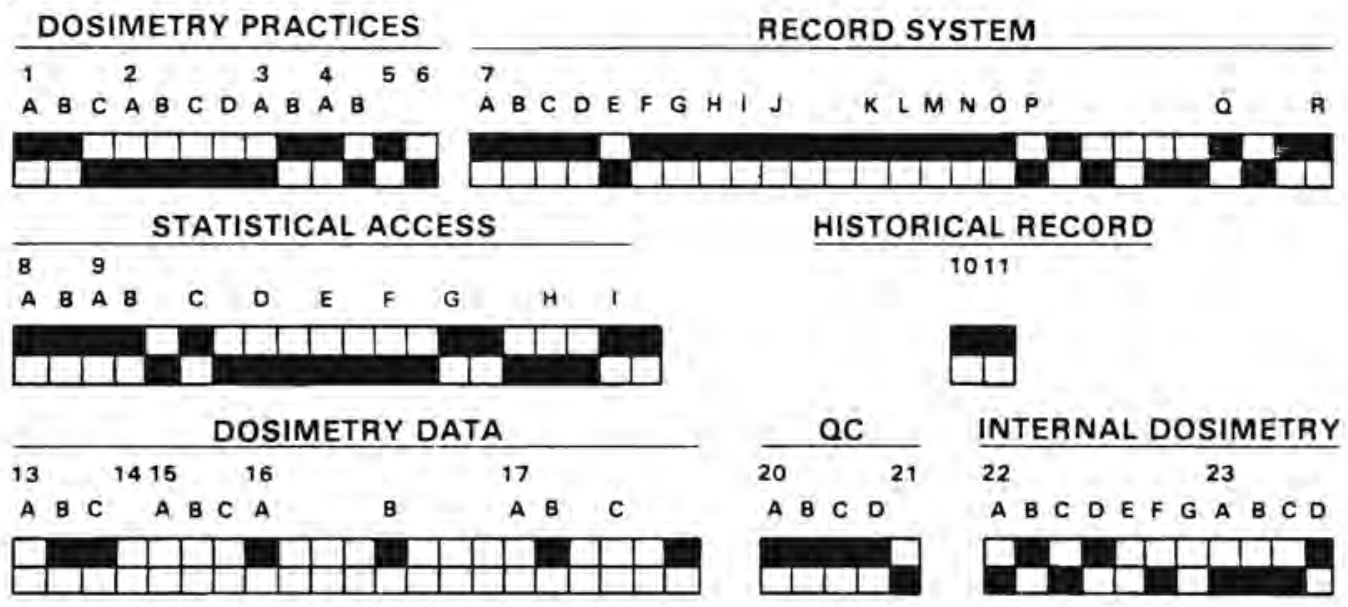

\section{SPECIFIC QUESTIONS AND ANSWERS}
\#12. MANUAL CHAPTERS
\#17. A. NONPENETRATION DOSE AT - NOT APPLICABLE
B. PEnetrating dose at - $1 \mathrm{~cm}$
\#18. A. THERMAL NEUTRON QUALITY FACTOR -
B. FAST NEUTRON QUALITY FACTOR-
C. TOTAL NEUTRON QUALITY FACTOR - 10

\#19. NONPENETRATING FILTER \#1.

$\# 2$ -

PENETRATING FILTER $\begin{aligned} & \# 1 . \\ & \# 2 . \\ & \# 3 .\end{aligned}$

LEGEND 
FACILITY - NN

TYPE - CONTRACTOR LABORATORY

DOSIMETRY VOLUME - MEDIUM

DOSIMETRY SERVICE - COMMERCIAL

SECTION I

DOSIMETRY DOCUMENTATION

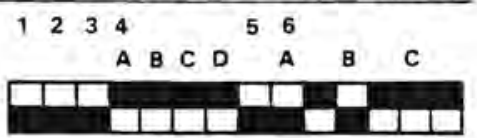

SECTION II

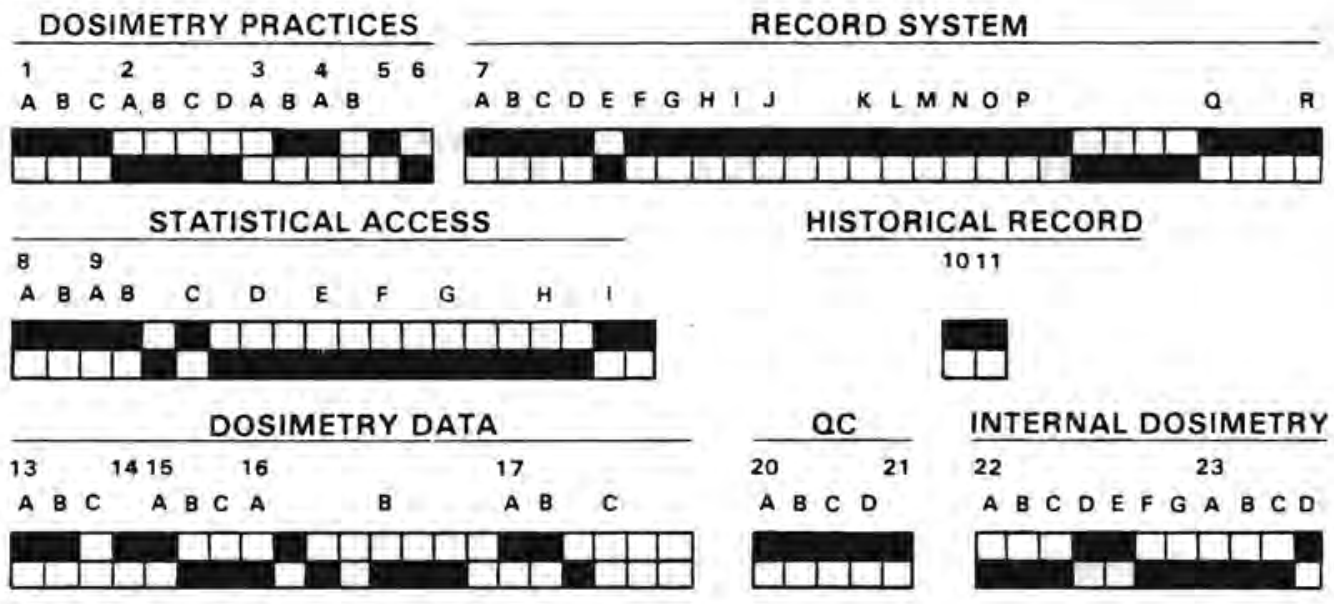

SPECIFIC QUESTIONS AND ANSWERS

\#12. MANUAL CHAPTERS

\#17. A. NONPENETRATION DOSE AT - $7 \mathrm{mg} / \mathrm{cm}^{2}$

B. PENETRATING DOSE AT - $1 \mathrm{~cm}$

\#18. A. THERMAL NEUTRON QUALITY FACTOR -

B. FAST NEUTRON QUALITY FACTOR-

C. TOTAL NEUTRON QUALITY FACTOR -

\#19. NONPENETRATING FILTER \#1.

$\$ 2$.

PENETRATING FILTER $\# 1$ -

$\# 2$ -

\#3-

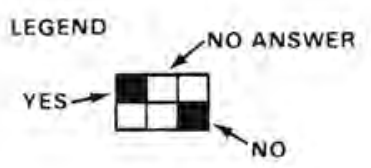


FACILITY - OO

TYPE - UNIVERSITY

DOSIMETRY VOLUME - MEDIUM

DOSIMETRY SERVICE - COMMERCIAL

\author{
SECTION I \\ DOSIMETRY DOCUMENTATION

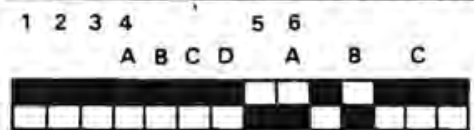

SECTION II

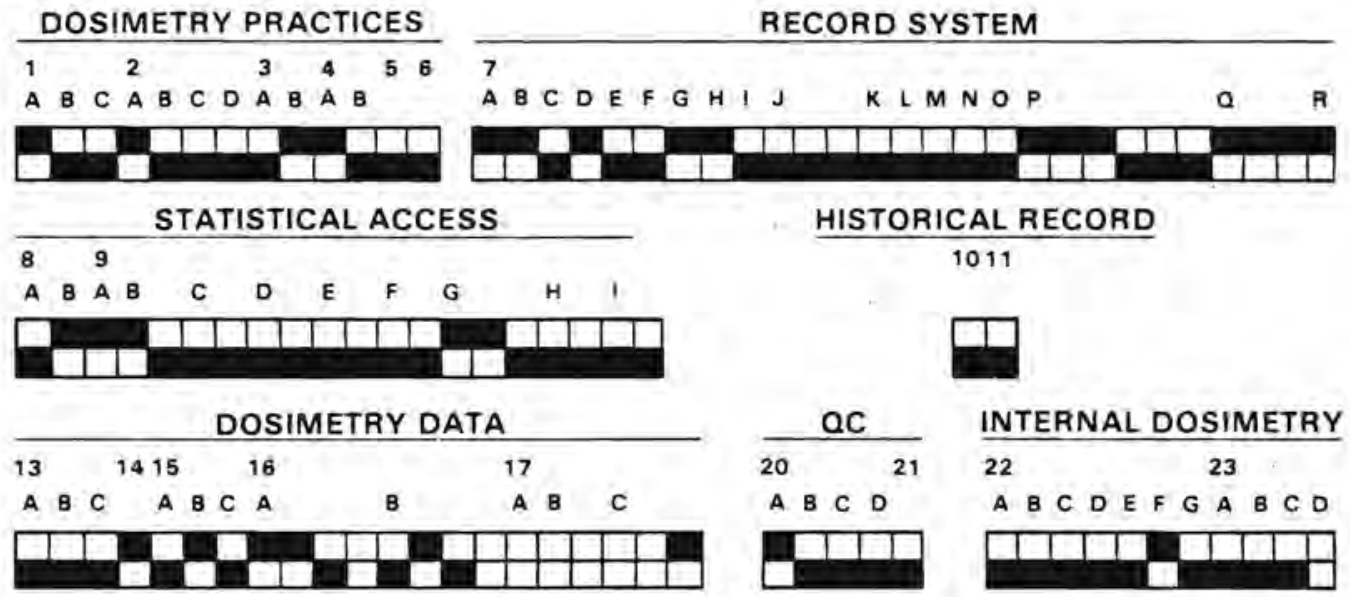

SPECIFIC QUESTIONS AND ANSWERS

\#12. MANUAL CHAPTERS

\#17. A. NONPENETRATION DOSE AT - UNKNOWN

B. PENETRATING DOSE AT - UNKNOWN

\#18. A. THERMAL NEUTRON QUALITY FACTOR -

B. FAST NEUTRON QUALITY FACTOR -

C. TOTAL NEUTRON QUALITY FACTOR $-1 \mathrm{~m} / \mathrm{cm}=2.6 \times 10^{4} / \mathrm{cm}^{2}$

\#19. NONPENETRATING FILTER \#1-22mg $\mathrm{cm}^{2}$ OF CHIP RETAINER $\# 2$.

PENETRATING

FILTER \#1- $375 \mathrm{mg} / \mathrm{cm}^{2}$ OF PLASTIC

$\# 2$ -

$\# 3$ -

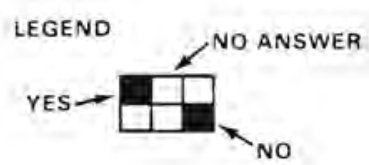


FACILITY - PP

TYPE - CONTRACTOR LABORATORY

DOSIMETRY VOLUME - SMALL

DOSIMETRY SERVICE - INHOUSE

\author{
SECTION I \\ DOSIMETRY DOCUMENTATION

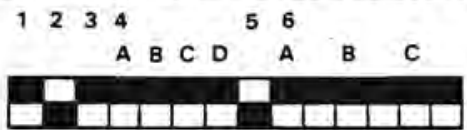

SECTION II

DOSIMETRY PRACTICES

$\begin{array}{llllll}1 & 2 & 3 & 4 & 5 & 6\end{array}$

A B C A B C D A B A B

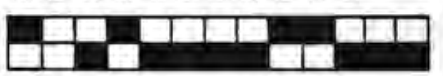

A BCDEFGHIJ KLMNOP

Q $\mathrm{B}$

STATISTICAL ACCESS

89

A B A B C D E F G
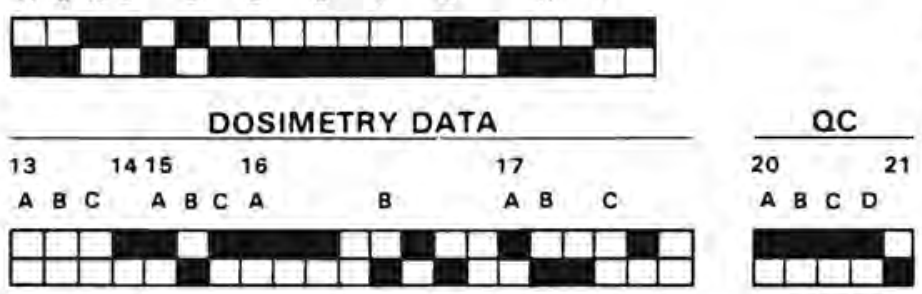

HISTORICAL RECORD

1011
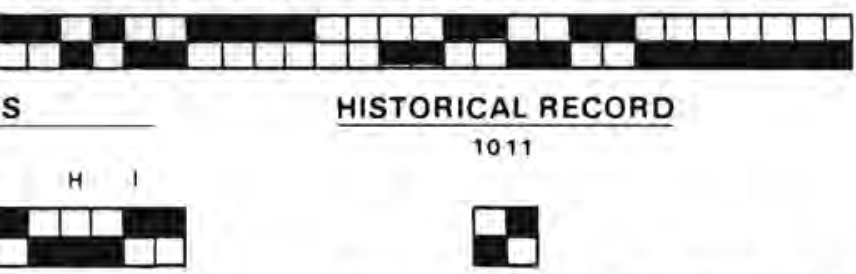

INTERNAL DOSIMETRY

A B C DEFG A B C D

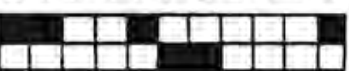

SPECIFIC QUESTIONS AND ANSWERS

\#12. MANUAL CHAPTERS

\#17. A. NONPENETRATION DOSE AT $-7 \mathrm{mg} / \mathrm{cm}^{2}$

B. PENETRATING DOSE AT - IN AIR

\#18. A. THERMAL NEUTRON QUALITY FACTOR - 10 CFR

B. FAST NEUTRON QUALITY FACTOR - 10 CFR

C. TOTAL NEUTRON QUALITY FACTOR - $10 \mathrm{CFR}$

\#19. NONPENETRATING FILTER \#1-25mg $/ \mathrm{cm}^{2}$ PLASTIC

$\# 2$ - $100 \mathrm{mg} / \mathrm{cm}^{2}$ PLASTIC

PENETRATING FILTER \#1- 400

\#2- -900

\#3.

LEGEND NO ANSWER 
FACILITY - QQ

TYPE - DOE LABORATORY

DOSIMETRY VOLUME - SMALL

DOSIMETRY SERVICE - COMMERCIAL

\section{SECTION I}

DOSIMETRY DOCUMENTATION

1234

A B C D

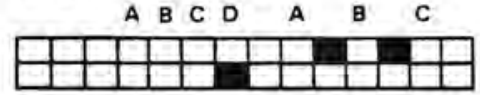

\section{SECTION II}

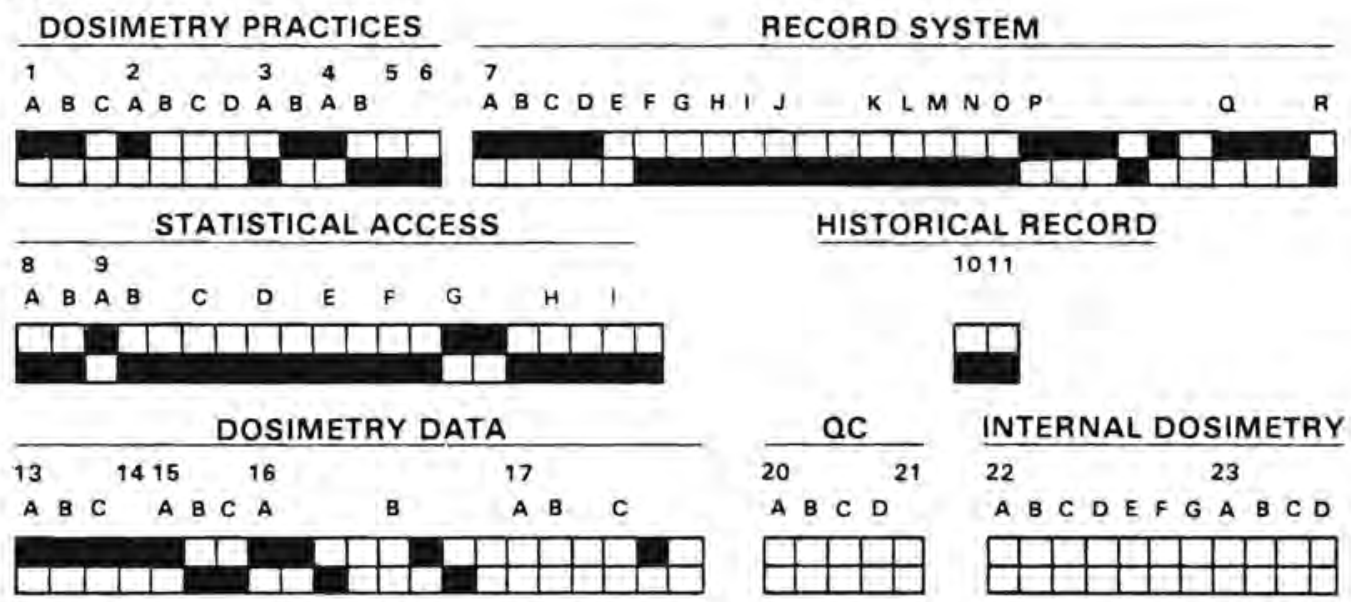

\section{SPECIFIC QUESTIONS AND ANSWERS}

\#12. 10 CFR 20

\#17. A. NONPENETRATION DOSE AT - UNKNOWN

B. PENETRATING DOSE AT - UNKNOWN

\#18. A. THERMAL NEUTRON QUALITY FACTOR -

B. FAST NEUTRON QUALITY FACTOR -

C. TOTAL NEUTRON QUALITY FACTOR

\#19. NONPENETRATING FILTER \#1

$$
\# 2 \text { - }
$$

PENETRATING

FILTER \#1 $\# 2$ $\# 3$.

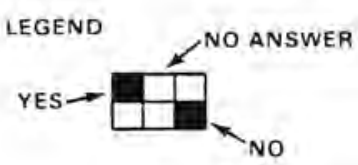




\section{DISTRIBUTION}

No. of

Copies

OFFSITE

A. A. Churm

DOE Patent Division

9800 South Cass Avenue

Argonne, IL 60439

5 E. J. Vallario

Assistant Chief/Manager

Health Physics Program

Operational and Environmental

Safety Division

U.S. Department of Energy

Washington, DC 20545

G. P. Dix

Director, Operational and

Environmental Safety Division

U.S. Department of Energy

Washington, DC 20545

H. C. Field

Chief, Policy, Measurements and Institutional Standards

Operational and Environmental Safety Division

U.S. Department of Energy

Washington, DC 20545

N. R. Glover

Leader, Performance Measurements Group

Policy, Measurements and Institutional Standards Branch

Operational and Environmental Safety Division

U.S. Department of Energy

Washington, DC 20545
No. of

Copies

E. N. Patigalia

Performance Measurements Group

Policy, Measurements and Institutional Standards Branch

Operational and Environmental Safety Division

U.S. Department of Energy

Washington, DC 20545

D. E. Patterson

Deputy Director

Operational and Environmental Safety Division

U.S. Department of Energy

Washington, DC 20545

D. M. Ross

Chief, Health Protection Branch

Operational and Environmental Safety Division

U.S. Department of Energy

Washington, DC 20545

C. A. Wood

Performance Measurements Group

Policy, Measurements and Institutional Standards Branch

Operational and Environmental Safety Division

U.S. Department of Energy

Washington, DC 20545

D. E. Jones

U.S. Department of Energy

550 Second Street

Idaho Falls, ID 83401

J. D. Moroney

U.S. Department of Energy

P.O. Box 14100

Las Vegas, NV 89114 
No. of

Copies

27 DOE Technical Information Center

B. C. Brooks

Licensee Operations Evaluations Branch

Division of Technical Support

Office of Management and Program Analys is

U.S. Nuclear Regulatory Commission Washington, D C 20555

C. L. Soden

Albuquerque Operations Office

P.0. Box 5400

Albuquerque, NM 87115

M. D. Voss

Ames Laboratory

I owa State University

Ames, IA 50011

W. E. Bleiler

Argonne National Laboratory

9700 S. Cass Avenue

Argonne, IL 60439

W. D. Fairman

Argonne National Laboratory

9700 S. Cass Avenue

Argonne, IL 60439

J. E. White

Bartlesville Energy

Technology Center

P.0. Box 1398

Bartlesville, OK 74003

F. X. Masse

Bates Linear Accellerator

P. 0: Box 95

Middleton, MA 01949

L. F. Phillips

Bldg. 535A

Brookhaven National Laboratory

Upton, NY 11978
No. of

Copies

G. J. Brisco

System Safety Development Center

EG\&G Idaho, Inc.

P.0. Box 1625

Idaho Falls, ID 83415

W. H. Millet

System Safety Development Center

EG\&G I daho, Inc.

P.0. Box 1625

Idaho Falls, ID 83415

R. D. Caldwe 11

Health Protection Dept.

Bldg. 735-A

E.I. DuPont de Nemours

Savannah River Plant

Aiken, SC 29801

R. S. Sanna

Environmental Measurements Laboratory

376 Hudson Street

New York, NY 10014

Fermi National Accelerator Laboratory

P. 0. Box 500

Batavia, IL 60510

H. R. Giorgio

Goodyear Atomic Corporation

P.0. Box 628

Piketon, $\mathrm{OH} 45661$

J. J. Thompson

Inhalation Toxicology Research Institute

P.0. Box 5890

Albuquerque, NM 87115

J. P. Lambert

Kansas State University

Ward Hall

Manhattan, KS 66506 
No. of

Copies

J. Takahashi

Laboratory of Nuclear Medicine

900 Veteran Avenue

Los Angeles, CA 90024

F. P. Miknis

Laramie Energy Technology Center

P.0. Box 3395

University Station

Laramie, WY 82070

J. B. McCaslin

Lawrence Berkeley Laboratory

Berkeley, CA 94720

K. M. Oswald

Lawrence Livermore Laboratory

P.0. Box 45

Mercury, NV 89023

S. S. Hoots

Lawrence Livermore National Laboratory

P.0. Box 5505

Livermore, CA 94550

J. N. P. Lawrence

Los Alamos Scientific Laboratory

Los Alamos, NM 87545

Mason \& Hanger-Silas Mason Co.

Pantex Plant

P.0. Box 30020

Amaril10, TX 79177

C. L. Phillabaum

Monsanto Research Corp.

Mound Facility

Miamisburg, $\mathrm{OH} 45342$

M. W. Boback

National Lead Company of Ohio P.0. Box 39158

Cincinnati, $\mathrm{OH} \quad 45239$
No. of

Copies

T. A. Dugan

National Lead Company of Ohio

P.0. Box 39158

Cincinnati, $\mathrm{OH} \quad 45239$

J. D. Berger

Oak Ridge Associated Universities

P.0. Box 117

Oak Ridge, TN 37830

E. D. Gupton

Oak Ridge National Laboratory

P.0. Box $X$

Oak Ridge, TN 37830

J. G. Dare

Ohio State University

Office of Radiation Safety

410 West 10th Avenue

Columbus, $\mathrm{OH} 43210$

R. C. Baker

Paducah Gaseous Diffusion Plant

P. 0. Box 1410

Paducah, KY 42001

B. E. McDougal

Paducah Gaseous Diffusion Plant

P. 0. Box 1410

Paducah, KY 42001

J. N. Holliday

Pinellas Plant

P.0. Box 11508

St. Petersburg, FL 33733

H. J. Howe, Jr.

Princeton Plasma Physics Laboratory

P. 0. Box 451

Princeton, NJ 08544

P. 0. Ziemer

Purdue University

Radiological Control Office

W. Lafayette, IN 47907 
No. of

Copies

R. J. Tuttle

Rockwe 11 International

8900 DeSoto Avenue

Canoga Park, CA 91304

Rockwe 11 International

Energy Systems Group

P. 0. Box 464

Golden, CO 80401

G. Millard

Sandia National Laboratory

Albuquerque, NM 37185

D. Wright

Sandia National Laboratory

East Avenue

Livermore, CA 94550

E. B. Rickansrud

P.0. Box 4349

Stanford University

Stanford, CA 94305

J. C. Bailey

Union Carbide Corporation

Nuclear Division (ORGDP)

P.0. Box $P$

Oak Ridge, TN 37830

C. M. Hopper

Union Carbide Corporation

Nuclear Division (UCC-ND)

P.0. Box $Y$

Oak Ridge, TN 37830

University of California, Davis

Environmental Health Safety

TB-30

Davis, CA 95616

Laboratory of Radiobiology

University of California

San Francisco, CA 94143

A. P. Imad

Environmental Health \& Safety

University of Colorado

Boulder, CO 80309
No. of

Copies

E. A. Ulicny

Radiation Laboratory

University of Notre Dame

Notre Dame, IN 46556

Radiation Safety Office

University of Pittsburgh

Rm A-550 Crabtree Hall GSPH

Pittsburgh, PA 15201

E. B. Darden, Jr.

University of Tennessee

Comparative Animal Research

Laboratory

1299 Bethel Valley Road

Oak Ridge, TN 37830

G. R. Holeman

314 WNSL-West

Yale University

260 Whitney Avenue

New Haven, CT 06520

\section{ONSITE}

5 DOE Richland Operations Office

M. F. Mills

H. E. Ransom

J. L. Rhoades

M. W. Tiernan

G. R. Yesberger

24 Pacific Northwest Laboratory

L. G. Faust

J. J. Fix (10)

W. A. Glass

G. R. Hoenes

H. W. Hunt

J. J. Jech

J. M. Selby

C. M. Unruh

V. L. Witherspoon

Technical Information (5)

Publishing Coordination (2) 